\title{
Generalized Kantorovich-Szász type operations involving Charlier polynomials
}

\author{
P. N. Agrawal ${ }^{a}$, Abhishek Kumara ${ }^{a}$, Aditi Kar Gangopadhyay ${ }^{a}$, Tarul Garg ${ }^{b}$ \\ ${ }^{a}$ Department of Mathematics, Indian Institute of Technology Roorkee, Roorkee-247667, India. \\ ${ }^{b}$ Department of Applied Science, The NorthCap University, Gurugram-122017, India.
}

\begin{abstract}
The purpose of this paper is to introduce a new kind of Kantorovich-Szász type operators based on Charlier polynomials and study its various approximation properties. We establish some local direct theorems, e.g., Voronovskaja type asymptotic theorem and an estimate of error by means of the Lipschitz type maximal function and the Peetre's K-functional. We also discuss the weighted approximation properties. Next, we construct a bivariate case of the above operators and study the degree of approximation with the aid of the complete and partial moduli of continuity. A Voronovskaja type asymptotic theorem and the order of convergence by considering the second order modulus of continuity are also proved. We define the associated Generalized Boolean Sum (GBS) operators and discuss the degree of approximation by using mixed modulus of smoothness for Bögel continuous and Bögel differentiable functions. Furthermore, by means of a numerical example it is shown that the proposed operators provide us a better approximation than the operators corresponding to the particular case $\wp=1$. We also illustrate the convergence of the bivariate operators and the associated GBS operators to a certain function and show that the GBS operators enable us a better error estimation than the bivariate operators using Matlab algorithm.
\end{abstract}

Keywords: Voronovskaya theorem, moduli of continuity, Peetre's K-functional, Bögel continuous function, Bögel differentiable function.

2020 MSC: 47A58.

(C)2021 All rights reserved.

\section{Introduction}

For $\varphi \in C(I)$, with $\mathrm{I}=[0,1]$, the classical Bernstein polynomial $\mathrm{B}_{\ell}(\varphi ; \varkappa)$ is defined by

$$
\mathrm{B}_{\ell}(\varphi ; \varkappa)=\sum_{j=0}^{\ell} \mathrm{p}_{\ell, j}(\varkappa) \varphi\left(\frac{j}{\ell}\right), \varkappa \in \mathrm{I},
$$

where $p_{\ell, j}(\varkappa)$ is the usual Bernstein basis function given by

$$
p_{\ell, j}(\varkappa)=\left(\begin{array}{l}
\ell \\
j
\end{array}\right) \varkappa^{j}(1-\varkappa)^{\ell-j}, \quad j=0,1, \ldots, \ell .
$$

\footnotetext{
*Corresponding author

Email addresses: pnappfma@gmail.com (P. N. Agrawal), anikk6887@gmail.com (Abhishek Kumar), aditifma@iitr.ac.in (Aditi Kar Gangopadhyay), tarulgarg@ncuindia.edu (Tarul Garg)
}

doi: $10.22436 /$ jnsa.014.04.04

Received: 2020-07-25 Revised: 2020-11-29 Accepted: 2020-12-02 
The classical Bernstein-Kantorovich operators are defined by

$$
\mathrm{K}_{\ell}(\varphi ; \varkappa)=(\ell+1) \sum_{j=0}^{\ell} p_{\ell, j}(\varkappa) \int_{\frac{j}{(\ell+1)}}^{\frac{(j+1)}{(\ell+1)}} \varphi(\mathrm{s}) \mathrm{d} s, \varkappa \in \mathrm{I}, \ell \in \mathbb{N},
$$

where $\varphi: I \rightarrow \mathbb{R}$ is an integrable function. The operators $K_{\ell}$ defined in (1.1) can also be expressed as follows:

$$
K_{\ell}(\varphi ; \varkappa)=(\ell+1) \sum_{j=0}^{\ell} p_{\ell, j}(\varkappa) \int_{0}^{1} \varphi\left(\frac{j+s}{\ell+1}\right) d s .
$$

For $\wp>0$, a new sequence of linear positive operators known as modified Bernstein-Kantorovich operators was given by Özarslan and Duman [18] as follows,

$$
\mathrm{K}_{\ell, \wp}(\varphi ; \varkappa)=\sum_{j=0}^{\ell} \mathrm{p}_{\ell, j}(\varkappa) \int_{0}^{1} \varphi\left(\frac{\mathrm{s}^{\wp}+\mathrm{j}}{\ell+1}\right) \mathrm{ds}, \varkappa \in \mathrm{I},
$$

wherein the author discussed some direct approximation theorems. It is clear that these operators include the classical Bernstein-Kantorovich operators $K_{\ell}$ defined by (1.1) for $\wp=1$. For $\varphi \in C\left(\mathbb{R}_{+}\right)$, the space of continuous functions on $\mathbb{R}_{+}:=[0, \infty)$, Varma and Taşdelen [22] defined the Szász type operators involving Charlier polynomials as follows:

$$
\mathcal{L}_{\ell}(\varphi ; \varkappa, d)=e^{-1}\left(1-\frac{1}{d}\right)^{(d-1) \ell \varkappa} \sum_{j=0}^{\infty} \frac{C_{j}^{(d)}(-(d-1) \ell \varkappa)}{j !} \varphi\left(\frac{j}{\ell}\right),
$$

where $d>1$ and the generating functions of the Charlier polynomials are given by

$$
e^{s}\left(1-\frac{s}{d}\right)^{v}=\sum_{j=0} C_{j}^{(d)}(v) \frac{s^{j}}{j !},|s|<d,
$$

$C_{j}^{(d)}(v)=\sum_{r=0}^{j}\left(\begin{array}{l}j \\ r\end{array}\right)(-v)_{r}\left(\frac{1}{d}\right)^{r}$ and $(n)_{0}=1,(n)_{i}=n(n+1) \cdots(n+i-1)$, for $i \geqslant 1$. We observe that if $\mathrm{d} \rightarrow \infty$ and $\varkappa$ is replaced with $\varkappa-\frac{1}{\ell}$ in (1.2), then these operators reduce to the Szász operators [20]. Verma and Taşdelen [22] studied the uniform convergence of the operators $\mathcal{L}_{\ell}(\varphi ; \varkappa, d)$ by applying Korovkin theorem on compact subsets of $\mathbb{R}_{+}$and obtained the order of approximation by using the classical modulus of continuity.

Motivated by the above work, for $\wp>0, \gamma>0$ and $\varphi \in C_{\gamma}\left(\mathbb{R}_{+}\right):=\left\{\phi \in C\left(\mathbb{R}_{+}\right):|\phi(s)| \leqslant M_{\phi}\left(1+s^{\gamma}\right)\right\}$, $M_{\phi}$ being a constant depending only on $\phi$, we define a generalized Kantorovich-Szász type operator based on the Charlier polynomials as follows:

$$
\mathcal{L}_{\ell, \wp}(\varphi ; \varkappa, d)=e^{-1}\left(1-\frac{1}{d}\right)^{(d-1) \ell \varkappa} \sum_{j=0}^{\infty} \frac{C_{j}^{(d)}(-(d-1) \ell \varkappa)}{j !} \int_{0}^{1} \varphi\left(\frac{s^{\wp}+j}{\ell+1}\right) d s, \quad d>1, \wp>0 .
$$

For $\wp=1$, the operators defined by (1.3) include the Kantorovich type modification of the Szász type operators based on Charlier polynomials studied by Varma and Taşdelen [22].

The outline of the paper is as follows. We investigate the rate of approximation of the operators given by equation (1.3). We consider a bivariate case of these operators and discuss the degree of approximation. Also, we construct the associated GBS(generalized Boolean sum) operator of the bivariate operator defined by (4.1) and investigate the rate of approximation for the Bögel continuous and Bögel differentiable functions by means of the mixed modulus of smoothness. We study some numerical examples to illustrate the superiority of the proposed operators over the operators corresponding to the case $\wp=1$. Let $e_{i}(s)=s^{i}, i=0,1,2,3,4$. 
Lemma 1.1 ([22]). The operators given by (1.2) satisfy the following equalities:

(i) $\mathcal{L}_{\ell}\left(e_{0} ; \varkappa, \mathrm{d}\right)=1$;

(ii) $\mathcal{L}_{\ell}\left(e_{1} ; \varkappa, d\right)=\varkappa+\frac{1}{\ell}$;

(iii) $\mathcal{L}_{\ell}\left(e_{2} ; \varkappa, d\right)=\varkappa^{2}+\frac{\varkappa}{\ell}\left(3+\frac{1}{d-1}\right)+\frac{2}{\ell^{2}} ;$

(iv) $\mathcal{L}_{\ell}\left(e_{3} ; \varkappa, \mathrm{d}\right)=\varkappa^{3}+\frac{\varkappa^{2}}{\ell}\left(6+\frac{3}{\mathrm{~d}-1}\right)+\frac{2 \varkappa}{\ell^{2}}\left(\frac{1}{(\mathrm{~d}-1)^{2}}+\frac{3}{\mathrm{~d}-1}+5\right)+\frac{5}{\ell^{3}}$;

(v) $\mathcal{L}_{\ell}\left(e_{4} ; \varkappa, d\right)=\varkappa^{4}+\frac{\varkappa^{3}}{\ell}\left(10+\frac{6}{d-1}\right)+\frac{\varkappa^{2}}{\ell^{2}}\left(31+\frac{30}{d-1}+\frac{11}{(d-1)^{2}}\right)+\frac{\varkappa}{\ell^{3}}\left(37+\frac{31}{d-1}+\frac{20}{(d-1)^{2}}+\frac{6}{(d-1)^{3}}\right)+\frac{15}{\ell^{4}}$.

As a consequence of the above Lemma, we have following.

Lemma 1.2. For the operators $\mathcal{L}_{\ell, \wp}$ defined by equation (1.3), we obtain

(i) $\mathcal{L}_{\ell, \wp}\left(e_{0} ; \varkappa, d\right)=1$;

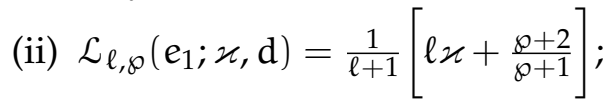

(iii) $\mathcal{L}_{\ell, \wp}\left(e_{2} ; \varkappa, d\right)=\frac{1}{(\ell+1)^{2}}\left[\ell^{2} \varkappa^{2}+\ell \varkappa\left(3+\frac{1}{d-1}+\frac{2}{\wp+1}\right)+\frac{2}{\wp+1}+\frac{1}{2 \wp+1}+2\right]$;

(iv) $\mathcal{L}_{\ell, \wp}\left(e_{3} ; \varkappa, \mathrm{d}\right)=\frac{1}{(\ell+1)^{3}}\left[\ell^{3} \varkappa^{3}+\ell^{2} \varkappa^{2}\left(6+\frac{3}{\mathrm{~d}-1}+\frac{3}{\wp+1}\right)+\ell \varkappa\left\{\frac{3}{2 \wp+1}+\frac{3}{\wp+1}\left(3+\frac{1}{\mathrm{~d}-1}\right)\right.\right.$

$\left.\left.+2\left(\frac{1}{(d-1)^{2}}+\frac{3}{d-1}+5\right)\right\}+\frac{6}{\wp+1}+\frac{1}{3 \wp+1}+\frac{3}{2 \wp+1}+5\right]$;

(v) $\mathcal{L}_{\ell, \wp}\left(e_{4} ; \varkappa, d\right)=\frac{1}{(\ell+1)^{4}}\left[\ell^{4} \varkappa^{4}+\ell^{3} \varkappa^{3}\left(\frac{6}{d-1}+\frac{4}{\wp+1}+10\right)+\ell^{2} \varkappa^{2}\left\{\frac{4}{\mathfrak{\wp}+1}\left(6+\frac{3}{d-1}\right)\right.\right.$

$\left.+\frac{30}{d-1}+\frac{11}{(d-1)^{2}}+\frac{6}{2 \wp+1}+31\right\}+\ell \varkappa\left\{\frac{6}{2 \wp+1}\left(3+\frac{1}{d-1}\right)+\frac{4}{\wp+1}\left(10+\frac{2}{d-1}\left(3+\frac{1}{d-1}\right)\right)+\frac{31}{d-1}\right.$

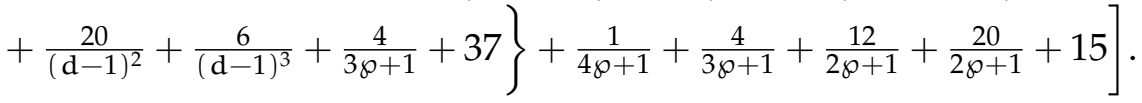

Consequently, by simple computations we obtain the following result.

Lemma 1.3. For the operators $\mathcal{L}_{\ell, \wp}(\cdot ; \varkappa, \mathrm{d})$, we have

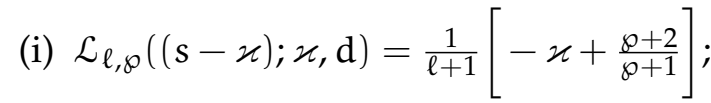

(ii) $\mathcal{L}_{\ell, \wp}\left((s-\varkappa)^{2} ; \varkappa, \mathrm{d}\right)=\frac{1}{(\ell+1)^{2}}\left[\varkappa^{2}+\varkappa\left\{\ell\left(3+\frac{1}{\mathrm{~d}-1}+\frac{2}{\wp+1}\right)-2(\ell+1) \frac{\wp+2}{\wp+1}\right\}+\frac{4 \wp^{2}+11 \wp+5}{(\wp+1)(2 \wp+1)}\right]$;

(iii) $\mathcal{L}_{\ell, \wp}\left((\mathrm{s}-\varkappa)^{4} ; \varkappa, \mathrm{d}\right)=\frac{1}{(\ell+1)^{4}}\left[\varkappa^{4}+\varkappa^{3}\left\{\ell^{3}\left(\frac{4}{\wp+1}+2\left(5+\frac{1}{\mathrm{~d}-1}\right)\right)+6 \ell(\ell+1)^{2}\left(\frac{2}{\wp+1}+\left(3+\frac{1}{\mathrm{~d}+1}\right)\right)\right.\right.$

$\left.-4 \ell^{2}(\ell+1)\left(\frac{3}{\wp+1}+\left(6+\frac{3}{d-1}\right)\right)-4 \ell(\ell+1)^{3}\right\}+\varkappa^{2}\left\{\ell^{2}\left(\frac{6}{2 \wp+1}+4\left(6+\frac{1}{d-1}\right)+\frac{1}{d-1}\left(30+\frac{11}{d-1}\right)+31\right)\right.$

$\left.+6 \ell(\ell+1)^{2}\left(\frac{1}{2 \wp+1}+2\left(1+\frac{1}{\wp+1}\right)\right)-4 \ell(\ell+1)\left(\frac{3}{2 \wp+1}+\frac{3}{\wp+1}\left(3+\frac{1}{d-1}\right)\right)+2\left(5+\frac{1}{d-1}\left(3+\frac{1}{d-1}\right)\right)\right\}$

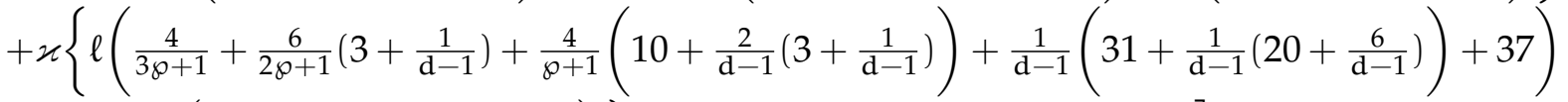

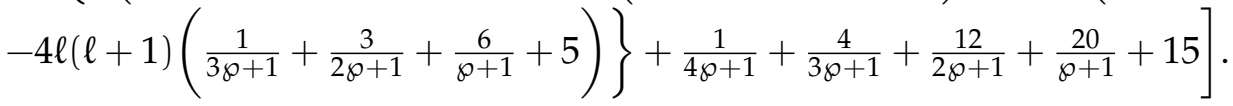

Remark 1.4. By using Lemma 1.3, we obtain

(i) $\lim _{\ell \rightarrow \infty} \ell \mathcal{L}_{\ell, \wp}((s-\varkappa) ; \varkappa, d)=-\varkappa+\frac{\wp+2}{\wp+1}$; 
(ii) $\lim _{\ell \rightarrow \infty} \ell \mathcal{L}_{\ell, \wp}\left((s-\varkappa)^{2} ; \varkappa, \mathrm{d}\right)=\frac{\mathrm{d} \varkappa}{\mathrm{d}-1}$;

(iii) $\lim _{\ell \rightarrow \infty} \ell^{2} \mathcal{L}_{\ell, \wp}\left((\mathrm{s}-\varkappa)^{4} ; \varkappa, \mathrm{d}\right)=\frac{3 \mathrm{~d}^{2} \varkappa}{(\mathrm{d}-1)^{2}}$.

Let $C_{B}\left(\mathbb{R}_{+}\right)$be the space of all real valued bounded continuous functions $\varphi$ on $\mathbb{R}$, endowed with the norm

$$
\|\varphi\|=\sup _{\varkappa \in \mathbb{R}_{+}}|\varphi(\varkappa)| .
$$

Lemma 1.5. For $\varphi \in \mathrm{C}_{\mathrm{B}}\left(\mathbb{R}_{+}\right)$and each $\varkappa \in \mathbb{R}_{+}$, we have

$$
\left|\mathcal{L}_{\ell, \wp}(\varphi ; \varkappa, d)\right| \leqslant\|\varphi\| .
$$

Proof. Applying Lemma 1.2, we have

$$
\left|\mathcal{L}_{\ell, \wp}(\varphi ; \varkappa, d)\right| \leqslant e^{-1}\left(1-\frac{1}{d}\right)^{(d-1) \ell \varkappa} \sum_{j=0}^{\infty} \frac{C_{j}^{(d)}(-(d-1) \ell \varkappa)}{j !} \int_{0}^{1}\left|\varphi\left(\frac{s^{\wp}+j}{\ell+1}\right)\right| d s \leqslant\|\varphi\| \mathcal{L}_{\ell, \wp}(1 ; \varkappa, d)=\|\varphi\|,
$$

which completes the proof.

\section{Approximation properties of $\mathcal{L}_{\ell, \wp}(\cdot ; \varkappa, \mathrm{d})$}

The following theorem shows that the operators $\mathcal{L}_{\ell, \wp}(. ; \varkappa, \mathrm{d})$ is an approximation process for continuous functions on $\mathbb{R}_{+}$.

Theorem 2.1. Let $\varphi \in \mathrm{C}_{\gamma}\left(\mathbb{R}_{+}\right)$. Then

$$
\lim _{\ell \rightarrow \infty} \mathcal{L}_{\ell, \wp}(\varphi ; \varkappa, d)=\varphi(\varkappa)
$$

holds uniformly on each compact subset of $\mathbb{R}_{+}$.

Proof. Applying Lemma 1.2, $\lim _{\ell \rightarrow \infty} \mathcal{L}_{\ell, \wp}(1 ; \varkappa, \mathrm{d})=1, \lim _{\ell \rightarrow \infty} \mathcal{L}_{\ell, \wp}(\mathrm{s} ; \varkappa, \mathrm{d})=\varkappa$ and $\lim _{\ell \rightarrow \infty} \mathcal{L}_{\ell, \wp}\left(\mathrm{s}^{2} ; \varkappa, \mathrm{d}\right)=\varkappa^{2}$, uniformly on each compact subset of $\mathbb{R}^{+}$, hence we obtain the desired result by using the well-known theorem given by Gadzhiev [13].

In what follows, let $\mu_{\ell, \wp, d, i}(\varkappa)=\mathcal{L}_{\ell, \wp}\left((s-\varkappa)^{i} ; \varkappa, d\right), i \in \mathbb{N} \cup\{0\}$.

Theorem 2.2. Let $\varphi \in \mathrm{C}_{\mathrm{B}}\left(\mathbb{R}_{+}\right)$. For the operators $\mathcal{L}_{\ell, \wp}(\varphi ; \varkappa, \mathrm{d})$ given by (1.3), there holds the estimation

$$
\left|\mathcal{L}_{\ell, \wp}(\varphi ; \varkappa, \mathrm{d})-\varphi(\varkappa)\right| \leqslant 2 \omega\left(\varphi ;\left(\mu_{\ell, \wp, \mathrm{d}, 2}(\varkappa)\right)^{\frac{1}{2}}\right) .
$$

Proof. Applying the property of modulus of continuity, for any $\delta>0$, we get

$$
\begin{aligned}
\left|\mathcal{L}_{\ell, \wp}(\varphi ; \varkappa, d)-\varphi(\varkappa)\right| & \leqslant e^{-1}\left(1-\frac{1}{d}\right)^{(d-1) \ell} \sum_{j=0}^{\infty} \frac{C_{j}^{(d)}(-(d-1) \ell \varkappa)}{j !} \int_{0}^{1}\left|\varphi\left(\frac{s^{\wp}+j}{\ell+1}\right)-\varphi(\varkappa)\right| d s \\
& \leqslant \omega(\varphi ; \delta)\left\{1+\frac{1}{\delta} e^{-1}\left(1-\frac{1}{d}\right)^{(d-1) \ell \varkappa} \sum_{j=0}^{\infty} \frac{C_{j}^{(d)}(-(d-1) \ell \varkappa)}{j !} \int_{0}^{1}\left|\frac{s^{\wp}+j}{\ell+1}-\varkappa\right| d s\right\} .
\end{aligned}
$$

Applying the Cauchy-Schwarz inequality and Lemma 1.2, we have

$$
e^{-1}\left(1-\frac{1}{d}\right)^{(d-1) \ell \varkappa} \sum_{j=0}^{\infty} \frac{C_{j}^{(d)}(-(d-1) \ell \varkappa)}{j !} \int_{0}^{1}\left|\frac{s^{\wp}+j}{\ell+1}-\varkappa\right| d s
$$




$$
\leqslant\left(e^{-1}\left(1-\frac{1}{d}\right)^{(d-1) \ell \varkappa} \sum_{j=0}^{\infty} \frac{C_{j}^{(d)}(-(d-1) \ell \varkappa)}{j !} \int_{0}^{1}\left(\frac{s^{\wp}+j}{\ell+1}-\varkappa\right)^{2} d s\right)^{\frac{1}{2}}=\left(\mu_{\ell, \wp, d, 2}(\varkappa)\right)^{\frac{1}{2}} .
$$

Hence,

$$
\left|\mathcal{L}_{\ell, \wp}(\varphi ; \varkappa, \mathrm{d})-\varphi(\varkappa)\right| \leqslant \omega(\varphi ; \delta)\left\{1+\frac{1}{\delta}\left(\mu_{\ell, \wp, \mathrm{d}, 2}(\varkappa)\right)^{\frac{1}{2}}\right\}=2 \omega\left(\varphi ;\left(\mu_{\ell, \wp, \mathrm{d}, 2}(\varkappa)\right)^{\frac{1}{2}}\right),
$$

on choosing $\delta:=\left\{\mu_{\ell, \wp, \mathrm{d}, 2}(\varkappa)\right\}^{\frac{1}{2}}$. This completes the proof.

Theorem 2.3. Let $\varphi \in \mathrm{C}_{\gamma}\left(\mathbb{R}_{+}\right)$. If $\varphi^{\prime \prime}$ exists at a point $\varkappa \in \mathbb{R}_{+}$, then

$$
\lim _{\ell \rightarrow \infty} \ell\left(\mathcal{L}_{\ell, \wp}(\varphi ; \varkappa, d)-\varphi(\varkappa)\right)=\left(-\varkappa+\frac{\wp+2}{\wp+1}\right) \varphi^{\prime}(\varkappa)+\frac{1}{2}\left(\frac{d \varkappa}{d-1}\right) \varphi^{\prime \prime}(\varkappa) .
$$

Further, the limit in (2.1) holds uniformly on each compact subset $[a, b]$ of $\mathbb{R}_{+}, 0 \leqslant a<b<\infty$.

Proof. By using Taylor's expansion of $\varphi$, we have

$$
\varphi(\mathrm{s})=\varphi(\varkappa)+\varphi^{\prime}(\varkappa)(\mathrm{s}-\varkappa)+\frac{1}{2} \varphi^{\prime \prime}(\varkappa)(\mathrm{s}-\varkappa)^{2}+(\mathrm{s}-\varkappa)^{2} \varepsilon(\mathrm{s}, \varkappa),
$$

where the function $\varepsilon(s, \varkappa) \rightarrow 0$, as $s \rightarrow \varkappa$. Operating by $\mathcal{L}_{\ell, \wp}(\cdot ; \varkappa, \mathrm{d})$ on both sides of the equality (2.2), we obtain

$$
\mathcal{L}_{\ell, \wp}(\varphi ; \varkappa, \mathrm{d})-\varphi(\varkappa)=\varphi^{\prime}(\varkappa) \mathcal{L}_{\ell, \wp}(\mathrm{s}-\varkappa ; \varkappa, \mathrm{d})+\frac{\varphi^{\prime \prime}(\varkappa)}{2} \mathcal{L}_{\ell, \wp}\left((\mathrm{s}-\varkappa)^{2} ; \varkappa, \mathrm{d}\right)+\mathcal{L}_{\ell, \wp}\left(\varepsilon(\mathrm{s}, \varkappa)(\mathrm{s}-\varkappa)^{2} ; \varkappa, \mathrm{d}\right) .
$$

Hence,

$$
\begin{aligned}
\lim _{\ell \rightarrow \infty} \ell\left(\mathcal{L}_{\ell, \wp}(\varphi ; \varkappa, \mathrm{d})-\varphi(\varkappa)\right)= & \varphi^{\prime}(\varkappa) \lim _{\ell \rightarrow \infty} \ell \mathcal{L}_{\ell, \wp}(\mathrm{s}-\varkappa ; \varkappa, \mathrm{d})+\frac{\varphi^{\prime \prime}(\varkappa)}{2} \lim _{\ell \rightarrow \infty} \ell \mathcal{L}_{\ell, \wp}\left((\mathrm{s}-\varkappa)^{2} ; \varkappa, \mathrm{d}\right) \\
& +\lim _{\ell \rightarrow \infty} \ell \mathcal{L}_{\ell, \wp}\left(\varepsilon(s, \varkappa)(\mathrm{s}-\varkappa)^{2} ; \varkappa, \mathrm{d}\right) .
\end{aligned}
$$

Applying Remark 1.4, we get

$$
\begin{aligned}
\lim _{\ell \rightarrow \infty} \ell\left(\mathcal{L}_{\ell, \wp}(\varphi ; \varkappa, \mathrm{d})-\varphi(\varkappa)\right)= & \left(-\varkappa+\frac{\wp+2}{\wp+1}\right) \varphi^{\prime}(\varkappa)+\frac{1}{2}\left(\frac{\mathrm{d} \varkappa}{\mathrm{d}-1}\right) \varphi^{\prime \prime}(\varkappa) \\
& +\lim _{\ell \rightarrow \infty} \ell \mathcal{L}_{\ell, \wp}\left(\varepsilon(\mathrm{s}, \varkappa)(\mathrm{s}-\varkappa)^{2} ; \varkappa, \mathrm{d}\right) .
\end{aligned}
$$

For the last term of the right side of (2.3), using Cauchy-Schwarz inequality, we are led to

$$
\left|\ell \mathcal{L}_{\ell, \wp}\left(\varepsilon(s, \varkappa)(s-\varkappa)^{2} ; \varkappa, d\right)\right| \leqslant \ell\left(\mathcal{L}_{\ell, \wp}\left(\varepsilon^{2}(s, \varkappa) ; \varkappa, d\right)\right)^{\frac{1}{2}}\left(\mathcal{L}_{\ell, \wp}\left((s-\varkappa)^{4} ; \varkappa, d\right)\right)^{\frac{1}{2}} .
$$

We observe that $\varepsilon^{2}(s, \varkappa) \in \mathrm{C}_{\alpha}\left(\mathbb{R}_{+}\right)$, for some $\alpha>0$, and $\varepsilon^{2}(s, \varkappa) \rightarrow 0$, as $s \rightarrow \varkappa$, hence, by Theorem 2.1, $\lim _{\ell \rightarrow \infty} \mathcal{L}_{\ell, \wp}\left(\varepsilon^{2}(s, \varkappa) ; \varkappa, d\right)=\varepsilon^{2}(\varkappa, \varkappa)=0$.

Further, using Remark $1.4, \lim _{\ell \rightarrow \infty} \ell\left(\mathcal{L}_{\ell, \wp}\left((s-\varkappa)^{4} ; \varkappa, d\right)\right)^{\frac{1}{2}}$ is finite. Hence,

$$
\lim _{\ell \rightarrow \infty} \ell\left(\mathcal{L}_{\ell, \wp}\left(\varepsilon(s, \varkappa)(s-\varkappa)^{2} ; \varkappa, d\right)\right)=0,
$$

Finally, consideration of (2.3) and (2.4) completes the proof of (2.1). To prove the uniformity assertion, it is sufficient to remark that the limits in (2.3) and (2.4) hold uniformly in $\varkappa \in[a, b] \subset \mathbb{R}^{+}$.

For $\varphi \in \tilde{\mathrm{C}}_{\mathrm{B}}\left(\mathbb{R}_{+}\right):=\left\{\varphi \in \mathrm{C}_{\mathrm{B}}\left(\mathbb{R}_{+}\right): \varphi\right.$ is uniformly continuous on $\left.\mathbb{R}_{+}\right\}$, the Peetre's K-functional is defined by

$$
K(\varphi ; \delta)=\inf _{f \in C_{B}^{2}\left(\mathbb{R}_{+}\right)}\left\{\|\varphi-f\|+\delta\left\|f^{\prime \prime}\right\|\right\}, \delta>0,
$$

where $C_{B}^{2}\left(\mathbb{R}_{+}\right)=\left\{f \in C_{B}\left(\mathbb{R}_{+}\right): f^{\prime}, f^{\prime \prime} \in C_{B}\left(\mathbb{R}_{+}\right)\right\}$. By [10, p.177, Theorem 2.4] there exists an absolute 
constant $M$ such that

$$
\mathrm{K}(\varphi, \delta) \leqslant M \omega_{2}(\varphi ; \sqrt{\delta}),
$$

where $\omega_{2}(\varphi ; \sqrt{\delta})$ is the second-order modulus of continuity of $\varphi$ on $\mathbb{R}_{+}$. The usual modulus of continuity of $\varphi \in \tilde{\mathrm{C}}_{\mathrm{B}}\left(\mathbb{R}_{+}\right)$is defined as

$$
\omega(\varphi, \delta)=\sup _{0<|h| \leqslant \delta} \sup _{\varkappa \in \mathbb{R}_{+}}|\varphi(\varkappa+h)-\varphi(\varkappa)| .
$$

Theorem 2.4. Let $\varphi \in \tilde{\mathrm{C}}_{\mathrm{B}}\left(\mathbb{R}_{+}\right)$. Then for every $\varkappa \in \mathbb{R}_{+}$, the following inequality holds

$$
\left|\mathcal{L}_{\ell, \wp}(\varphi ; \varkappa, \mathrm{d})-\varphi(\varkappa)\right| \leqslant M \omega_{2}\left(\varphi ; \frac{\beta_{\ell, \wp, \mathrm{d}}(\varkappa)}{2}\right)+\omega\left(\varphi ; \frac{1}{\ell+1}\left|-\varkappa+\frac{\wp+2}{\wp+1}\right|\right),
$$

where $M$ is an absolute positive constant and

$$
\beta_{\ell, \wp, \mathrm{d}}^{2}(\varkappa)=\frac{1}{2}\left\{\mu_{\ell, \wp, \mathrm{d}, 2}(\varkappa)+\frac{1}{(\ell+1)^{2}}\left(-\varkappa+\frac{\wp+2}{\wp+1}\right)^{2}\right\} .
$$

Proof. First, we define an auxiliary operator as follows:

$$
\overline{\mathcal{L}}_{\ell, \wp}(\varphi ; \varkappa, \mathrm{d})=\mathcal{L}_{\ell, \wp}(\varphi ; \varkappa, \mathrm{d})+\varphi(\varkappa)-\varphi\left(\frac{\ell \varkappa}{\ell+1}+\frac{\wp+2}{(\ell+1)(\wp+1)}\right) .
$$

Applying Lemma 1.2, it is seen that

$$
\overline{\mathcal{L}}_{\ell, \wp}\left(e_{0} ; \varkappa, \mathrm{d}\right)=1 \text { and } \overline{\mathcal{L}}_{\ell, \wp}((\mathrm{s}-\varkappa) ; \varkappa, \mathrm{d})=0 .
$$

Let $h \in C_{B}^{2}\left(\mathbb{R}_{+}\right)$. From Taylor's formula, we may write

$$
h(s)=h(\varkappa)+(s-\varkappa) h^{\prime}(\varkappa)+\int_{\varkappa}^{s}(s-v) h^{\prime \prime}(v) d v .
$$

From the above equality, in view of (2.7) and (2.6) we get

$$
\overline{\mathcal{L}}_{\ell, \wp}(\mathrm{h}(\mathrm{s}) ; \varkappa, \mathrm{d})=\mathcal{L}_{\ell, \wp}\left(\int_{\varkappa}^{s}(s-v) \mathrm{h}^{\prime \prime}(v) \mathrm{d} v ; \varkappa, \mathrm{d}\right)-\int_{\varkappa}^{\frac{\ell \varkappa}{\ell+1}+\frac{\wp+2}{(\ell+1)(\wp+1)}}\left(\frac{\ell \varkappa}{\ell+1}+\frac{\wp+2}{(\ell+1)(\wp+1)}-v\right) h^{\prime \prime}(v) \mathrm{d} v,
$$

which implies that

$$
\begin{aligned}
\left|\overline{\mathcal{L}}_{\ell, \wp}(\mathrm{h} ; \varkappa, \mathrm{d})-\mathrm{h}(\varkappa)\right| \leqslant & \mathcal{L}_{\ell, \wp}\left(\left|\int_{\varkappa}^{s}\right| s-v|| \mathrm{h}^{\prime \prime}(v)|\mathrm{d} v| ; \varkappa, \mathrm{d}\right) \\
& +\left|\int_{\varkappa}^{\frac{\ell \varkappa}{\ell+1}+\frac{\wp+2}{(\ell+1)(\wp+1)}}\right| \frac{\ell \varkappa}{\ell+1}+\frac{\wp+2}{(\ell+1)(\wp+1)}-v|| \mathrm{h}^{\prime \prime}(v)|\mathrm{d} v| .
\end{aligned}
$$

Now, using the fact that

$$
\left|\int_{\varkappa}^{s}\right| s-v|| h^{\prime \prime}(v)|d v| \leqslant \frac{\left\|h^{\prime \prime}\right\|}{2}(s-\varkappa)^{2},
$$

it follows that

$$
\mathcal{L}_{\ell, \wp}\left(\left|\int_{\ell}^{s}\right| s-v \| h^{\prime \prime}(v)|d v| ; \ell, d\right) \leqslant \frac{\left\|h^{\prime \prime}\right\|}{2} \mathcal{L}_{\ell, \wp}\left((s-\varkappa)^{2} ; \varkappa, d\right) .
$$

Thus using (2.8), we obtain

$$
\left|\int_{\varkappa}^{\ell \varkappa+1}+\frac{\wp+2}{\ell+1)(\wp+1)}\right| \frac{\ell \varkappa}{\ell+1}+\frac{\wp+2}{(\ell+1)(\wp+1)}-v|| \mathrm{h}^{\prime \prime}(v)|\mathrm{d} v| \leqslant \frac{\left\|\mathrm{h}^{\prime \prime}\right\|}{2}\left(\frac{\ell \varkappa}{\ell+1}+\frac{\wp+2}{(\ell+1)(\wp+1)}-\varkappa\right)^{2}
$$




$$
=\frac{\left\|h^{\prime \prime}\right\|}{2(\ell+1)^{2}}\left(-\ell+\frac{\wp+2}{\wp+1}\right)^{2}
$$

Hence,

$$
\begin{aligned}
\left|\overline{\mathcal{L}}_{\ell, \wp}(\mathrm{h} ; \varkappa, \mathrm{d})-\mathrm{h}(\varkappa)\right| & \leqslant \frac{\left\|\mathrm{h}^{\prime \prime}\right\|}{2}\left\{\mathcal{L}_{\ell, \wp}\left((\mathrm{s}-\varkappa)^{2} ; \varkappa, \mathrm{d}\right)+\frac{1}{(\ell+1)^{2}}\left(-\varkappa+\frac{\wp+2}{\wp+1}\right)^{2}\right\} \\
& \leqslant \frac{\left\|\mathrm{h}^{\prime \prime}\right\|}{2}\left[\mu_{\ell, \wp, \mathrm{d}, 2}(\varkappa)+\frac{1}{(\ell+1)^{2}}\left(-\varkappa+\frac{\wp+2}{\wp+1}\right)^{2}\right] .
\end{aligned}
$$

From equation (2.6), for every $\varphi \in \tilde{\mathrm{C}}_{\mathrm{B}}\left(\mathbb{R}_{+}\right)$we have

$$
\left|\overline{\mathcal{L}}_{\ell, \wp}(\varphi ; \varkappa, \mathrm{d})\right| \leqslant 3\|\varphi\| .
$$

Hence from (2.6), (2.9), and (2.10), for $\varphi \in \tilde{\mathrm{C}}_{\mathrm{B}}\left(\mathbb{R}_{+}\right)$and any $\mathrm{h} \in \mathrm{C}_{\mathrm{B}}^{2}\left(\mathbb{R}_{+}\right)$, we obtain

$$
\begin{aligned}
\left|\mathcal{L}_{\ell, \wp}(\varphi ; \varkappa, \mathrm{d})-\varphi(\varkappa)\right| \leqslant & \left|\overline{\mathcal{L}}_{\ell, \wp}(\varphi ; \varkappa, \mathrm{d})-\varphi(\varkappa)+\varphi\left(\frac{\ell \varkappa}{\ell+1}+\frac{\wp+2}{(\ell+1)(\wp+1)}\right)-\varphi(\varkappa)\right| \\
\leqslant & \left|\overline{\mathcal{L}}_{\ell, \wp}(\varphi-\mathrm{h} ; \varkappa, \mathrm{d})\right|+\left|\overline{\mathcal{L}}_{\ell, \wp}(\mathrm{h} ; \varkappa, \mathrm{d})-\mathrm{h}(\varkappa)\right|+|\mathrm{h}(\varkappa)-\varphi(\varkappa)| \\
& +\left|\varphi\left(\frac{\ell \varkappa}{\ell+1}+\frac{\wp+2}{(\ell+1)(\wp+1)}\right)-\varphi(\varkappa)\right| \\
\leqslant & 4\|\varphi-\mathrm{h}\|+\frac{\left\|\mathrm{h}^{\prime \prime}\right\|}{2}\left[\mu_{\ell, \wp, \mathrm{d}, 2}(\varkappa)+\frac{1}{(\ell+1)^{2}}\left(-\varkappa+\frac{\wp+2}{\wp+1}\right)^{2}\right] \\
& +\omega\left(\varphi ; \frac{1}{\ell+1}\left|-\varkappa+\frac{\wp+2}{\wp+1}\right|\right) \\
\leqslant & 4\|\varphi-\mathrm{h}\|+\left\|\mathrm{h}^{\prime \prime}\right\| \beta_{\ell, \wp, \mathrm{d}}^{2}(\varkappa)+\omega\left(\varphi ; \frac{1}{\ell+1}\left|-\varkappa+\frac{\wp+2}{\wp+1}\right|\right) .
\end{aligned}
$$

Now, taking the infimum on the right hand side over all $h \in C_{B}^{2}\left(\mathbb{R}_{+}\right)$, we get

$$
\left|\mathcal{L}_{\ell, \wp}(\varphi ; \varkappa, \mathrm{d})-\varphi(\varkappa)\right| \mid \leqslant 4 \mathrm{~K}\left(\varphi ; \frac{\beta_{\ell, \wp, \mathrm{d}}^{2}(\varkappa)}{4}\right)+\omega\left(\varphi ; \frac{1}{\ell+1}\left|-\varkappa+\frac{\wp+2}{\wp+1}\right|\right) .
$$

Hence, in view of (2.5), we obtain

$$
\left|\mathcal{L}_{\ell, \wp}(\varphi ; \varkappa, \mathrm{d})-\varphi(\varkappa)\right| \leqslant M \omega_{2}\left(\varphi ; \frac{\beta_{\ell, \wp, \mathrm{d}}(\varkappa)}{2}\right)+\omega\left(\varphi ; \frac{1}{\ell+1}\left|-\varkappa+\frac{\wp+2}{\wp+1}\right|\right),
$$

which completes the proof.

For $\alpha \in(0,2]$, we consider the following Lipschitz-type space [17]: $\operatorname{Lip}_{M}^{(0,2]}(\alpha)=\left\{\varphi \in C\left(\mathbb{R}_{+}\right)\right.$: $\left.|\varphi(s)-\varphi(\varkappa)| \leqslant M \frac{|s-\varkappa|^{\alpha}}{(s+\varkappa)^{\frac{\alpha}{2}}} ; s \in \mathbb{R}_{+}, \varkappa>0\right\}$, where $M$ is a positive constant depending only on $\varphi$.

Theorem 2.5. Let $\varphi \in \operatorname{Lip}_{M}^{(0,2]}(\alpha), 0<\alpha \leqslant 2$. Then, for all $\varkappa \in(0, \infty)$, we have

$$
\left|\mathcal{L}_{\ell, \wp}(\varphi ; \varkappa, \mathrm{d},)-\varphi(\varkappa)\right| \leqslant M\left(\frac{\mu_{\ell, \vartheta, \mathrm{d}, 2}(\varkappa)}{\varkappa}\right)^{\frac{\alpha}{2}} .
$$

Proof. First, we prove the theorem for the case $\alpha=2$. Then, for $\varphi \in \operatorname{Lip}_{M}^{(0,2]}(\alpha)$, we have

$$
\left|\mathcal{L}_{\ell, \wp}(\varphi ; \varkappa, d)-\varphi(\varkappa)\right| \leqslant e^{-1}\left(1-\frac{1}{d}\right)^{(d-1) \ell \varkappa} \sum_{j=0}^{\infty} \frac{C_{j}^{(d)}(-(d-1) \ell \varkappa)}{j !} \int_{0}^{1}\left|\varphi\left(\frac{s^{\wp}+j}{\ell+1}\right)-\varphi(\varkappa)\right| d s
$$




$$
\leqslant M e^{-1}\left(1-\frac{1}{d}\right)^{(d-1) \ell \varkappa} \sum_{j=0}^{\infty} \frac{C_{j}^{(d)}(-(d-1) \ell \varkappa)}{j !} \int_{0}^{1} \frac{\left(\frac{s^{\ell}+j}{\ell+1}-\varkappa\right)^{2}}{\left(\frac{s^{\ell}+j}{\ell+1}+\varkappa\right)} d s .
$$

Using the fact that $\frac{1}{\left(\frac{\mathrm{s}^{\ell}+j}{\ell+1}+\varkappa\right)} \leqslant \frac{1}{\varkappa}, \forall \mathbf{j}=0,1,2, \ldots$, and the Cauchy-Schwarz inequality, we have

$$
\begin{aligned}
\left|\mathcal{L}_{\ell, \wp}(\varphi ; \varkappa, \mathrm{d})-\varphi(\varkappa)\right| & \leqslant \frac{M}{\varkappa}\left\{e^{-1}\left(1-\frac{1}{\mathrm{~d}}\right)^{(\mathrm{d}-1) \ell \varkappa} \sum_{j=0}^{\infty} \frac{C_{j}^{(\mathrm{d})}(-(\mathrm{d}-1) \mathrm{mx})}{j !} \int_{0}^{1}\left(\frac{\mathrm{s}^{\wp}+j}{\ell+1}-\varkappa\right)^{2} \mathrm{ds}\right\} \\
& \leqslant \frac{M}{\varkappa} \mathcal{L}_{\ell, \wp}\left((s-\varkappa)^{2} ; \varkappa, \mathrm{d}\right) \leqslant M\left(\frac{\mu_{\ell, \wp, \mathrm{d}, 2}(\varkappa)}{\varkappa}\right) .
\end{aligned}
$$

Hence the result is proved for $\alpha=2$. Now, let us prove the theorem for the case $0<\alpha<2$. Applying the Hölder inequality with $p=\frac{2}{\alpha}$ and $q=\frac{2}{2-\alpha}$, in view of Lemma 1.2, we find that

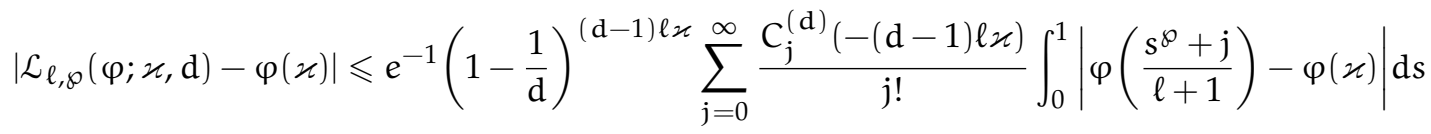

$$
\begin{aligned}
& \leqslant e^{-1}\left(1-\frac{1}{d}\right)^{(d-1) \ell \varkappa} \sum_{j=0}^{\infty} \frac{C_{j}^{(d)}(-(d-1) \ell \varkappa)}{j !}\left(\int_{0}^{1}\left|\varphi\left(\frac{s^{\wp}+j}{\ell+1}\right)-\varphi(\varkappa)\right|^{\frac{2}{\alpha}} d s\right)^{\frac{\alpha}{2}} \\
& \leqslant\left\{e^{-1}\left(1-\frac{1}{d}\right)^{(d-1) \ell \varkappa} \sum_{j=0}^{\infty} \frac{C_{j}^{(d)}(-(d-1) \ell \varkappa)}{j !} \int_{0}^{1}\left|\varphi\left(\frac{s^{\wp}+j}{\ell+1}\right)-\varphi(\varkappa)\right|^{\frac{2}{\alpha}} d s\right\}^{\frac{\alpha}{2}} \\
& \times\left\{e^{-1}\left(1-\frac{1}{d}\right)^{(d-1) \ell \varkappa} \sum_{j=0}^{\infty} \frac{C_{j}^{(d)}(-(d-1) \ell \varkappa)}{j !}\right\}^{\frac{2-\alpha}{2}} \\
& =\left\{e^{-1}\left(1-\frac{1}{d}\right)^{(d-1) \ell \varkappa} \sum_{j=0}^{\infty} \frac{C_{j}^{(d)}(-(d-1) \ell \varkappa)}{j !} \int_{0}^{1}\left|\varphi\left(\frac{s^{\wp}+j}{\ell+1}\right)-\varphi(\varkappa)\right|^{\frac{2}{\alpha}} d s\right\}^{\frac{\alpha}{2}} \\
& \leqslant M\left\{e^{-1}\left(1-\frac{1}{d}\right)^{(d-1) \ell \varkappa} \sum_{j=0}^{\infty} \frac{C_{j}^{(d)}(-(d-1) \ell \varkappa)}{j !} \int_{0}^{1} \frac{\left(\frac{s^{\ell}+j}{\ell+1}-\varkappa\right)^{2}}{\left(\frac{s^{\mathcal{P}}+j}{\ell+1}+\varkappa\right)} d s\right\}^{\frac{\alpha}{2}} \\
& \leqslant \frac{M}{\varkappa^{\frac{\alpha}{2}}}\left(e^{-1}\left(1-\frac{1}{d}\right)^{(d-1) \ell \varkappa} \sum_{j=0}^{\infty} \frac{C_{j}^{(d)}(-(d-1) \ell \varkappa)}{j !} \int_{0}^{1}\left(\frac{s^{\wp}+j}{\ell+1}-\varkappa\right)^{2} d s\right)^{\frac{\alpha}{2}} \\
& \leqslant M\left\{\frac{\mu_{\ell, \wp, \mathrm{d}, 2}(\varkappa)}{\varkappa}\right\}^{\frac{\alpha}{2}} \text {, }
\end{aligned}
$$

which leads us to the desired result.

Next, we study a local direct estimate for the operators defined in (1.3) by applying the Lipschitz-type maximal function of order $\alpha$ given by Lenze [16] as

$$
\tilde{\omega}_{\alpha}(\varphi, \varkappa)=\sup _{s \neq \varkappa, s \in \mathbb{R}_{+}} \frac{|\varphi(s)-\varphi(\varkappa)|}{|s-\varkappa|^{\alpha}}, \varkappa \in \mathbb{R}_{+} \text {and } \alpha \in(0,1] .
$$

Theorem 2.6. Let $\varphi \in \tilde{\mathrm{C}}_{\mathrm{B}}\left(\mathbb{R}_{+}\right)$and $0<\alpha \leqslant 1$. Then, for all $\varkappa \in \mathbb{R}_{+}$, we have

$$
\left|\mathcal{L}_{\ell, \wp}(\varphi ; \varkappa, d, 2)-\varphi(\varkappa)\right| \leqslant \tilde{\omega}_{\alpha}(\varphi, \varkappa)\left(\mu_{\ell, \wp, d, 2}(\varkappa)\right)^{\frac{\alpha}{2}} .
$$

Proof. In view of (2.11), we have

$$
|\varphi(s)-\varphi(\varkappa)| \leqslant \tilde{\omega}_{\alpha}(\varphi, \varkappa)|s-\varkappa|^{\alpha}
$$

and hence 


$$
\left|\mathcal{L}_{\ell, \wp}(\varphi ; \varkappa, \mathrm{d})-\varphi(\varkappa)\right| \leqslant \mathcal{L}_{\ell, \wp}(|\varphi(s)-\varphi(\varkappa)| ; \varkappa, \mathrm{d}) \leqslant \tilde{\omega}_{\alpha}(\varphi, \varkappa) \mathcal{L}_{\ell, \wp}\left(|s-\varkappa|^{\alpha} ; \varkappa, \mathrm{d}\right) .
$$

Now, applying the Hölder's inequality with $p=\frac{2}{\alpha}$ and $q=\frac{2}{2-\alpha}$, in view of Lemma 1.2, we obtain

$$
\left|\mathcal{L}_{\ell, \wp}(\varphi ; \varkappa, \mathrm{d})-\varphi(\varkappa)\right| \leqslant \tilde{\omega}_{\alpha}(\varphi, \varkappa) \mathcal{L}_{\ell, \wp}\left((\mathrm{s}-\varkappa)^{2} ; \varkappa, \mathrm{d}\right)^{\frac{\alpha}{2}} \leqslant \tilde{\omega}_{\alpha}(\varphi, \varkappa)\left(\mu_{\ell, \wp, \mathrm{d}, 2}(\varkappa)\right)^{\frac{\alpha}{2}} .
$$

Thus, the proof is completed.

Let $\phi(\varkappa)=\sqrt{\varkappa(1+\varkappa)}$ and $\varphi \in \tilde{\mathrm{C}}_{\mathrm{B}}\left(\mathbb{R}_{+}\right)$, then the unified Ditzian-Totik modulus $\omega_{\phi^{\lambda}}(\varphi, \eta), 0 \leqslant \lambda \leqslant 1$ is defined as

$$
\omega_{\phi^{\lambda}}(\varphi, \eta)=\sup _{0 \leqslant h \leqslant \eta} \sup _{\varkappa \pm \frac{h \phi^{\lambda}(\varkappa)}{2} \in \mathbb{R}_{+}}\left|\varphi\left(\varkappa+\frac{h \phi^{\lambda}(\varkappa)}{2}\right)-\varphi\left(\varkappa-\frac{h \phi^{\lambda}(\varkappa)}{2}\right)\right|
$$

and the appropriate K-functional is given by

$$
K_{\phi^{\lambda}}(\varphi, \eta)=\inf _{f \in W_{\lambda}}\left\{\|\varphi-f\|+\eta\left\|\phi^{\lambda} f^{\prime}\right\|\right\},
$$

where $W_{\lambda}=\left\{f: f \in A C_{\text {loc }}\left(\mathbb{R}_{+}\right),\left\|\phi^{\lambda} f^{\prime}\right\|<\infty\right\}$ and $A C_{\text {loc }}\left(\mathbb{R}_{+}\right)$denotes the space of locally absolutely continuous function on $\mathbb{R}_{+}$.

From [11], it is known that $\omega_{\phi^{\lambda}}(\varphi, \eta) \sim K_{\phi^{\lambda}}(\varphi, \eta)$, i.e., there exists a constant $M>0$ such that

$$
M^{-1} \omega_{\phi^{\lambda}}(\varphi, \eta) \leqslant K_{\phi^{\lambda}}(\varphi, \eta) \leqslant M \omega_{\phi^{\lambda}}(\varphi, \eta) .
$$

Theorem 2.7. Let $\varphi \in \tilde{\mathrm{C}}_{\mathrm{B}}\left(\mathbb{R}_{+}\right)$. Then for each $\varkappa \in \mathbb{R}_{+}$and sufficiently large $\ell$, there holds the inequality

$$
\left|\mathcal{L}_{\ell, \wp}(\varphi ; \varkappa, \mathrm{d})-\varphi(\varkappa)\right| \leqslant \mathrm{C} \omega_{\phi^{\lambda}}\left(\varphi, \frac{\phi^{1-\lambda}(\varkappa)}{\sqrt{\ell}}\right),
$$

where $\mathrm{C}$ is some constant independent of $\varphi$ and $\ell$.

Proof. From the definition of $K_{\phi^{\lambda}}(\varphi, \eta)$, for a fixed $\lambda, \ell$, and $\varkappa \in \mathbb{R}_{+}$we can choose $f=f_{\ell, \varkappa, \lambda} \in W_{\lambda}$ such that

$$
\|\varphi-f\|+\frac{\phi^{1-\lambda}(\varkappa)}{\sqrt{\ell}}\left\|\phi^{\lambda} f^{\prime}\right\| \leqslant 2 K_{\phi^{\lambda}}\left(\varphi, \frac{\phi^{1-\lambda}}{\sqrt{\ell}}\right) .
$$

From the representation

$$
f(s)=f(\varkappa)+\int_{\varkappa}^{s} f^{\prime}(u) d u
$$

it follows that

$$
\left|\mathcal{L}_{\ell, \wp}(f ; \varkappa, d)-f(\varkappa)\right| \leqslant \mathcal{L}_{\ell, \wp}\left(\left|\int_{\varkappa}^{s} f^{\prime}(u) d u\right| ; \varkappa, d\right) .
$$

Now, applying the Hölder's inequality,

$$
\left|\int_{\varkappa}^{s} f^{\prime}(u) d u\right| \leqslant\left\|\phi^{\lambda} f^{\prime}\right\|\left|\int_{\varkappa}^{s} \frac{1}{\phi^{\lambda}(u)} d u\right| \leqslant\left\|\phi^{\lambda} f^{\prime}\right\||s-\varkappa|^{1-\lambda}\left|\int_{\varkappa}^{s} \frac{1}{\phi(u)} d u\right|^{\lambda} .
$$

Now,

$$
\left|\int_{\varkappa}^{s} \frac{d u}{\phi(u)}\right| \leqslant \int_{\varkappa}^{s} \frac{d u}{\sqrt{u}}\left(\frac{1}{\sqrt{1+\varkappa}}+\frac{1}{\sqrt{1+s}}\right) \text { and }\left|\int_{\varkappa}^{t} \frac{d u}{\sqrt{u}}\right| \leqslant \frac{2|s-\varkappa|}{\sqrt{\varkappa}}
$$

hence

$$
\left|\int_{\varkappa}^{s} \frac{d u}{\phi(u)}\right| \leqslant \frac{2^{\lambda}|s-\varkappa|^{\lambda}}{\varkappa^{\frac{\lambda}{2}}}\left|\frac{1}{\sqrt{1+\varkappa}}+\frac{1}{\sqrt{1+s}}\right| \leqslant \frac{2^{\lambda}|s-\varkappa|^{\lambda}}{\varkappa^{\frac{\lambda}{2}}}\left((1+s)^{-\frac{\lambda}{2}}+(1+\varkappa)^{-\frac{\lambda}{2}}\right) .
$$


Thus

$$
\left|\mathcal{L}_{\ell, \wp}(\mathrm{f} ; \varkappa, \mathrm{d})-\mathrm{f}(\varkappa)\right| \leqslant \frac{2^{\lambda}}{\varkappa^{\frac{\lambda}{2}}(1+\varkappa)^{\frac{\lambda}{2}}}\left\|\phi^{\lambda} \mathrm{f}^{\prime}\right\| \mathcal{L}_{\ell, \wp}(|\mathrm{s}-\varkappa| ; \varkappa, \mathrm{d})+\frac{2^{\lambda}\left\|\phi^{\lambda} \mathrm{f}^{\prime}\right\|}{\varkappa^{\frac{\lambda}{2}}} \mathcal{L}_{\ell, \wp}\left(\frac{|\mathrm{s}-\varkappa|}{(1+s)^{\frac{\lambda}{2}}} ; \varkappa, \mathrm{d}\right) .
$$

Applying Cauchy-Schwarz inequality

$$
\mathcal{L}_{\ell, \wp}(|s-\varkappa| ; \varkappa, d) \leqslant\left(\mathcal{L}_{\ell, \wp}\left((s-\varkappa)^{2} ; \varkappa, d\right)\right)^{\frac{1}{2}} \leqslant \frac{\sqrt{M} \phi(\varkappa)}{\sqrt{(\ell+1)}} .
$$

Now,

$$
\mathcal{L}_{\ell, \wp}\left(\frac{|s-\varkappa|}{(1+s)^{\frac{\lambda}{2}}} ; \varkappa, d\right) \leqslant\left(\mathcal{L}_{\ell, \wp}\left((s-\varkappa)^{2} ; \varkappa, d\right)\right)^{\frac{1}{2}}\left(\mathcal{L}_{\ell, \wp}\left((1+s)^{-\lambda} ; \varkappa, d\right)\right)^{\frac{1}{2}} \leqslant M_{1} \frac{\sqrt{M} \phi(\varkappa)}{\sqrt{(\ell+1)}}(1+\varkappa)^{-\frac{\lambda}{2}} .
$$

Hence

$$
\left|\mathcal{L}_{\ell, \wp}(f ; \varkappa, d)-f(\varkappa)\right| \leqslant \frac{2^{\lambda}\left\|\phi^{\lambda} f^{\prime}\right\|}{\phi^{\lambda}(\varkappa)} \frac{\sqrt{M} \phi(\varkappa)}{\sqrt{(\ell+1)}}+\frac{2^{\lambda}\left\|\phi^{\lambda} f^{\prime}\right\|}{\varkappa^{\frac{\lambda}{2}}} \frac{M_{1} \sqrt{M}}{\sqrt{\ell+1}}(1+\varkappa)^{-\frac{\lambda}{2}} \leqslant M_{2} \frac{\left\|\phi^{\lambda} f^{\prime}\right\| \phi^{1-\lambda}(\varkappa)}{\sqrt{\ell+1}} .
$$

Thus, we have

$$
\begin{aligned}
\left|\mathcal{L}_{\ell, \wp}(\varphi ; \varkappa, d)-\varphi(\varkappa)\right| & \leqslant\left|\mathcal{L}_{\ell, \wp}(\varphi-f ; \varkappa, d)\right|+\left|\mathcal{L}_{\ell, \wp}(f ; \varkappa, d)-f(\varkappa)\right|+|f(\varkappa)-\varphi(\varkappa)| \\
& \leqslant 2\|\varphi-f\|+\frac{M_{2}\left\|\phi^{\lambda} f^{\prime}\right\| \phi^{1-\lambda}(\varkappa)}{\sqrt{\ell+1}} .
\end{aligned}
$$

Hence

$$
\left|\mathcal{L}_{\ell, \wp}(\varphi ; \varkappa, \mathrm{d})-\varphi(\varkappa)\right| \leqslant M_{3} \mathrm{~K}_{\phi^{\lambda}}\left(\varphi ; \frac{\phi^{1-\lambda}(\varkappa)}{\sqrt{\ell+1}}\right) \leqslant \mathrm{C} \omega_{\phi^{\lambda}}\left(\varphi ; \frac{\phi^{1-\lambda}(\varkappa)}{\sqrt{\ell+1}}\right),
$$

in view of (2.12). This completes the proof.

Now, we consider the space $C_{\gamma}\left(\mathbb{R}_{+}\right)$with $\gamma=2$. Assume that the space $C_{2}\left(\mathbb{R}_{+}\right)$is normed by $\|\varphi\|_{2}=$ $\sup _{\varkappa \in \mathbb{R}_{+}} \frac{|\varphi(\varkappa)|}{\left(\left(1+\varkappa^{2}\right)\right.}, \forall \varphi \in \mathrm{C}_{2}\left(\mathbb{R}_{+}\right)$. Further, let

$$
\mathrm{C}_{2}^{*}\left(\mathbb{R}^{+}\right)=\left\{\varphi \in \mathrm{C}_{2}\left(\mathbb{R}^{+}\right): \lim _{\varkappa \rightarrow \infty} \frac{|\varphi(\varkappa)|}{1+\varkappa^{2}}<\infty\right\} .
$$

Theorem 2.8. Let $\varphi \in \mathrm{C}_{2}\left(\mathbb{R}_{+}\right)$. Then, we have

$$
\left\|\mathcal{L}_{\ell \wp}(\varphi ; \cdot, d)-\varphi\right\|_{C[0, c]} \leqslant 4 M_{\varphi}\left(1+c^{2}\right) \mu_{\ell, \wp, d}(c)+2 \omega\left(\varphi ; \sqrt{\mu_{\ell, \wp, d}(c)},[0,1+c]\right),
$$

where $\mu_{\ell, \wp, \mathrm{d}}(\mathrm{c})=\sup _{\varkappa \in[0, \mathrm{c}]}\left(\mu_{\ell, \wp, \mathrm{d}, 2}(\varkappa)\right)$.

Proof. Let $\varkappa \in[0, c]$ and $s>c+1$. Then $s-\varkappa>1$, hence

$$
\begin{aligned}
|\varphi(s)-\varphi(\varkappa)| \leqslant M_{\varphi}\left(2+s^{2}+\varkappa^{2}\right) & =M_{\varphi}\left\{2+2 \varkappa^{2}+(s-\varkappa)^{2}+2 \varkappa(s-\varkappa)\right\} \\
& \leqslant M_{\varphi}(s-\varkappa)^{2}\left(3+2 \varkappa+2 \varkappa^{2}\right) \leqslant 4 M_{\varphi}\left(1+\varkappa^{2}\right)(s-\varkappa)^{2} .
\end{aligned}
$$

Applying [15], for $\varkappa \in[0, c]$ and $s \in[0, c+1]$ we have

$$
|\varphi(s)-\varphi(\varkappa)| \leqslant \omega(\varphi ;|s-\varkappa|,[0, c+1]) \leqslant \omega(\varphi ; \delta,[0, c+1])\left(1+\frac{|s-\varkappa|}{\delta}\right), \delta>0 .
$$


Thus, from (2.13) and (2.14) for all $\varkappa \in[0, c]$ and $s \geqslant 0$, we obtain

$$
|\varphi(s)-\varphi(\varkappa)| \leqslant 4 M_{\varphi}\left(1+\varkappa^{2}\right)(s-\varkappa)^{2}+\omega(\varphi ; \delta,[0, c+1])\left(1+\frac{|s-\varkappa|}{\delta}\right) .
$$

Hence applying the operators $\mathcal{L}_{\ell, \wp, \mathrm{d}}$ on the above and using Cauchy-Schwarz inequality, we get

$$
\begin{aligned}
\left|\mathcal{L}_{\ell, \wp}(\varphi ; \varkappa, \mathrm{d})-\varphi(\varkappa)\right| \leqslant & 4 \mathrm{M}_{\varphi}\left(1+\varkappa^{2}\right) \mathcal{L}_{\ell, \wp, \mathrm{d}}\left((\mathrm{s}-\varkappa)^{2} ; \varphi, \mathrm{d}\right) \\
& +\omega(\varphi ; \delta,[0, \mathrm{c}+1])\left(1+\frac{1}{\delta} \mathcal{L}_{\ell, \wp, \mathrm{d}}(|s-\varkappa| ; \varkappa, \mathrm{d})\right) \\
\leqslant & 4 \mathrm{M}_{\varphi}\left(1+\varkappa^{2}\right) \mu_{\ell, \wp, \mathrm{d}, 2}(\varkappa)+\omega(\varphi ; \delta,[0, \mathrm{c}+1])\left(1+\frac{1}{\delta} \sqrt{\mu_{\ell \wp, \mathrm{d}, 2}(\varkappa)}\right) \\
\leqslant & 4 M_{\varphi}\left(1+\mathrm{c}^{2}\right) \mu_{\ell, \wp, \mathrm{d}}(\mathrm{c})+\omega(\varphi ; \delta,[0, \mathrm{c}+1])\left(1+\frac{1}{\delta} \sqrt{\mu_{\ell, \wp, \mathrm{d}}(\mathrm{c})}\right) .
\end{aligned}
$$

Now, choosing $\delta=\sqrt{\mu_{\ell, \ell, d}(\mathrm{c})}$, we get the desired result.

Theorem 2.9. Let $\mathrm{g} \in \mathrm{C}_{2}^{*}\left(\mathbb{R}_{+}\right)$. Then, we have

$$
\lim _{\ell \rightarrow \infty}\left\|\mathcal{L}_{\ell, \wp}(\varphi ; \cdot, d)-\varphi\right\|_{2}=0 .
$$

Proof. Following [13], we know that it is sufficient to verify the following three conditions

$$
\lim _{\ell \rightarrow \infty}\left\|\mathcal{L}_{\ell, \wp}\left(s^{j} ; j, d\right)-\varkappa^{j}\right\|_{2}=0, j=0,1,2 .
$$

Since $\mathcal{L}_{\ell, \wp}(1 ; \varkappa, d)=1$, the condition in (2.15) is true for $j=0$. By Lemma 1.3, we have

$$
\left\|\mathcal{L}_{\ell, \wp}(s ; \cdot, d)-\varkappa\right\|_{2} \leqslant \sup _{\varkappa \geqslant 0} \frac{1}{1+\varkappa^{2}}\left|-\frac{\varkappa}{\ell+1}+\frac{\wp+2}{(\ell+1)(\wp+1))}\right| \leqslant \frac{\wp+2}{(\ell+1)(\wp+1))} .
$$

Thus, $\lim _{\ell \rightarrow \infty}\left\|\mathcal{L}_{\ell, \wp}(s ; \cdot, d)-\varkappa\right\|_{2}=0$. Similarly, we get

$$
\begin{aligned}
\left\|\mathcal{L}_{\ell, \wp}\left(\mathrm{s}^{2} ;, \mathrm{d}\right)-\varkappa^{2}\right\|_{2}= & \sup _{\varkappa \geqslant 0} \frac{1}{1+\varkappa^{2}}\left|\mathcal{L}_{\ell}^{(\wp)}\left(\mathrm{s}^{2} ; \ell, \mathrm{d}\right)-\varkappa^{2}\right| \\
= & \sup _{\varkappa \geqslant 0} \frac{1}{1+\varkappa^{2}} \mid \frac{\ell^{2} \varkappa^{2}}{(\ell+1)^{2}}+\frac{\ell \varkappa}{(\ell+1)^{2}}\left(3+\frac{1}{\mathrm{~d}-1}+\frac{2}{\wp+1}\right) \\
& +\frac{1}{(\ell+1)^{2}}\left(\frac{2}{\wp+1}+\frac{1}{2 \wp+1}+2\right)-\varkappa^{2} \mid \\
\leqslant & \sup _{\varkappa \geqslant 0} \frac{1}{1+\varkappa^{2}} \mid-\frac{(1+2 \ell) \varkappa^{2}}{(\ell+1)^{2}}+\frac{\ell \varkappa}{(\ell+1)^{2}}\left(3+\frac{1}{\mathrm{~d}-1}+\frac{2}{\wp+1}\right) \\
& +\frac{1}{(\ell+1)^{2}}\left(\frac{2}{\wp+1}+\frac{1}{2 \wp+1}+2\right) \mid \\
\leqslant & \sup _{\varkappa \geqslant 0} \frac{\varkappa^{2}}{1+\varkappa^{2}} \frac{(1+2 \ell)}{(\ell+1)^{2}}+\sup _{\varkappa \geqslant 0} \frac{\varkappa}{1+\varkappa^{2}} \frac{\ell\left(3+\frac{1}{\mathrm{~d}-1}+\frac{2}{\wp+1}\right)}{(\ell+1)^{2}}+\sup _{\varkappa \geqslant 0} \frac{1}{1+\varkappa^{2}} \frac{\left(\frac{2}{\wp+1}+\frac{1}{2 \wp+1}+2\right)}{(\ell+1)^{2}} \\
\leqslant & \frac{1+2 \ell}{(\ell+1)^{2}}+\frac{\mathrm{m}\left(3+\frac{1}{\mathrm{~d}-1}+\frac{2}{\wp+1}\right)}{(\ell+1)^{2}}+\frac{\left(\frac{2}{\wp+1}+\frac{1}{2 \wp+1}\right)+2}{(\ell+1)^{2}},
\end{aligned}
$$

which implies that

$$
\lim _{\ell \rightarrow \infty}\left\|\mathcal{L}_{\ell, \wp}\left(s^{2} ; \cdot, d\right)-\varkappa^{2}\right\|_{2}=0
$$

This completes the proof. 


\section{Numerical results and discussions}

We present a graph and a numerical table to show the comparison in the rate of approximation between the operators $\mathcal{L}_{\ell, \wp}(\varphi ; \varkappa, \mathrm{d})$ and $\mathcal{L}_{\ell, 1}(\varphi ; \varkappa, \mathrm{d})$. For this purpose, we recall some definitions to see that which sequence converges faster than the other. Let $u=\left(u_{n}\right)$ and $v=\left(v_{n}\right)$ be sequences with limits $u_{0}$ and $v_{0}$ and $u \neq\left(u_{n}\right), v \neq\left(v_{n}\right)$ for all $n$ and $0<K<\infty$ then we have the following result:

$$
\lim _{n \rightarrow \infty} \frac{\left|v_{n}-v_{0}\right|}{\left|u_{n}-u_{0}\right|}= \begin{cases}0, & \text { if }\left(v_{n}\right) \text { converges faster than }\left(u_{n}\right), \\ K, & \text { if convergence of }\left(u_{n}\right) \text { and }\left(v_{n}\right) \text { are equivalent, } \\ \infty, & \text { if }\left(u_{n}\right) \text { converges faster than }\left(v_{n}\right)\end{cases}
$$

If $0<\mathrm{K}<1$, then we say that the convergence of $v$ is better than the convergence of $u$. If $1<\mathrm{K}<\infty$, then it is said that the convergence of $u$ is better than the convergence of $v$ (please see [21, Chapter-II]).

Example 3.1. Let $\varphi(\varkappa)$ and $\Phi_{\ell, \wp, \mathrm{d}}(\varkappa)$ be, respectively, given as follows;

$$
\varphi(\varkappa)=\left(\varkappa-\frac{1}{2}\right)\left(\varkappa-\frac{1}{3}\right)\left(\varkappa-\frac{1}{4}\right)\left(\varkappa-\frac{1}{5}\right) \text { and } \Phi_{\ell, \wp, \mathrm{d}}(\varkappa)=\frac{\left|\mathcal{L}_{\ell, \wp}(\varphi ; \varkappa, \mathrm{d})-\varphi(\varkappa)\right|}{\left|\mathcal{L}_{\ell, 1}(\varphi ; \varkappa, \mathrm{d})-\varphi(\varkappa)\right|} .
$$

Let us choose $\ell=20, \mathrm{~d}=2$, and $\wp=1.5,2,2.5$, and 3. The convergence of the operators generalized Kantorovich-Szász-Charlier $\mathcal{L}_{20,1}(\varphi ; \varkappa, d)$ and the generalized Kantorovich-Szász-Charlier operators $\mathcal{L}_{20, \wp}(\varphi ; \varkappa, \mathrm{d})$ to $\varphi(\varkappa)=\left(\varkappa-\frac{1}{2}\right)\left(\varkappa-\frac{1}{3}\right)\left(\varkappa-\frac{1}{4}\right)\left(\varkappa-\frac{1}{5}\right)$ is illustrated in Figure 1. In Table 1, we calculate the ratio $\Phi_{\ell, \wp, \mathrm{d}}(\varkappa)$ for different values of $\varkappa$ by choosing $\wp=1.5,2,2.5$, and 3, respectively, for $\ell=20, \mathrm{~d}=2$. We observe that $\Phi_{\ell, \wp, \mathrm{d}}(\varkappa)<1$, for $\ell=20$ and $\mathrm{d}=2$, at different values of $\varkappa$ and $\wp$, therefore the sequence $\mathcal{L}_{\ell, \wp}(\varphi ; \varkappa, d)$ yields better convergence to $\varphi(\varkappa)$ than the sequence $\mathcal{L}_{\ell, 1}(\varphi ; \varkappa, d)$.

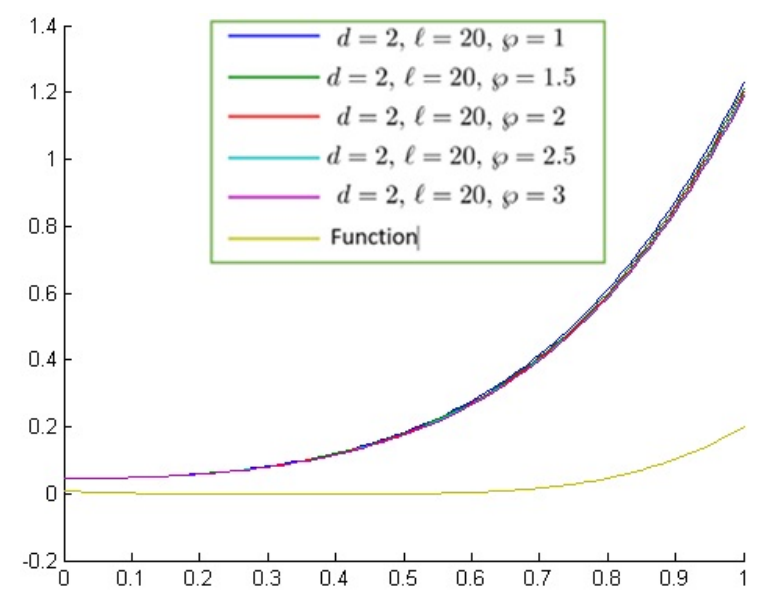

Figure 1: The convergence of operators $\mathcal{L}_{20, \wp}(\varphi ; \varkappa, d)$ to the function $\varphi(\varkappa)$.

Table 1: Ratio $\Phi_{\ell, \wp, \mathrm{d}}(\varkappa)$ at different values of $\varkappa$ and $\wp$.

\begin{tabular}{|c|c|c|c|c|c|c|}
\hline$\varkappa$ & 0.1 & 0.3 & 0.5 & 0.7 & 0.9 & 1 \\
\hline$\Phi_{20,1.5, \mathrm{~d}}(\varkappa)$ & 0.997821 & 0.9860406 & 0.982877 & 0.98257 & 0.982913 & 0.9829171 \\
\hline$\Phi_{20,2, \mathrm{~d}}(\varkappa)$ & 0.997821 & 0.978426 & 0.972032 & 0.9713914 & 0.9715659 & 0.9716284 \\
\hline$\Phi_{20,2.5, \mathrm{~d}}(\varkappa)$ & 0.995643 & 0.97208 & 0.964041 & 0.963329 & 0.9634228 & 0.9635365 \\
\hline$\Phi_{20,3, \mathrm{~d}}(\varkappa)$ & 0.995643 & 0.967005 & 0.9583333 & 0.9570871 & 0.95742 & 0.9575425 \\
\hline
\end{tabular}




\section{Bivariate construction of $\mathcal{L}_{\ell, \wp}(\cdot ; \varkappa, d)$}

For $\gamma_{1}, \gamma_{2}>0$, let

$$
\mathrm{C}_{\gamma_{1}, \gamma_{2}}\left(\mathbb{R}_{+}^{2}\right):=\left\{\psi \in \mathrm{C}\left(\mathbb{R}_{+}^{2}\right):\left|\psi\left(s_{1}, s_{2}\right)\right| \leqslant M_{\psi}\left(1+s_{1}^{\gamma_{1}}\right)\left(1+s_{2}^{\gamma_{2}}\right), \forall\left(s_{1}, s_{2}\right) \in \mathbb{R}_{+}^{2}\right\} .
$$

For $\varphi \in \mathrm{C}_{\gamma_{1}, \gamma_{2}}\left(\mathbb{R}_{+}^{2}\right)$, we define a bivariate extension of the operators given by (1.3) as follows:

$$
\begin{aligned}
\mathcal{L}_{\ell_{1}, \ell_{2}, \wp_{1}, \wp_{2}}\left(\varphi ; \varkappa_{1}, \varkappa_{2}, d_{1}, d_{2}\right)= & e^{-2} A_{\ell_{1}, \ell_{2}}\left(\varkappa_{1}, \varkappa_{2}, d_{1}, d_{2}\right) \sum_{j_{1}=0}^{\infty} \sum_{j_{2}=0}^{\infty} C_{\ell_{1}, \ell_{2}, j_{1}, j_{2}}\left(\varkappa_{1}, \varkappa_{2}, d_{1}, d_{2}\right) \\
& \times \int_{0}^{1} \int_{0}^{1} \varphi\left(\frac{s_{1}^{\wp_{1}}+j_{1}}{\ell_{1}+1}, \frac{s_{2}^{\wp_{2}}+j_{2}}{\ell_{2}+1}\right) d s_{1} d s_{2},
\end{aligned}
$$

where

and

$$
A_{\ell_{1}, \ell_{2}}\left(\varkappa_{1}, \varkappa_{2}, d_{1}, d_{2}\right)=\left(1-\frac{1}{d_{1}}\right)^{\left(d_{1}-1\right) \ell_{1} \varkappa_{1}}\left(1-\frac{1}{d_{2}}\right)^{\left(d_{2}-1\right) \ell_{2} \varkappa_{2}},
$$

$$
C_{\ell_{1}, \ell_{2}, j_{1}, j_{2}}\left(\varkappa_{1}, \varkappa_{2}, d_{1}, d_{2}\right)=\frac{C_{j_{1}}^{\left(d_{1}\right)}\left(-\left(d_{1}-1\right) \ell_{1} \varkappa_{1}\right)}{j_{1} !} \frac{C_{j_{2}}^{\left(d_{2}\right)}\left(-\left(d_{2}-1\right) \ell_{2} \varkappa_{2}\right)}{j_{2} !} .
$$

We examine the approximation properties and some results using the test functions

$$
e_{i, j}\left(s_{1}, s_{2}\right)=s_{1}^{i} s_{2}^{j}, i, j \in\{0,1,2,3,4\} \text { with } i+j \leqslant 4 \text {. }
$$

As a consequence of Lemma 1.2 and the definition (4.1), we obtain the following.

Lemma 4.1. For the bivariate operators $\mathcal{L}_{\ell_{1}, \ell_{2}, \wp_{1}, \wp_{2}}\left(\varphi ; \varkappa_{1}, \varkappa_{2}, \mathrm{~d}_{1}, \mathrm{~d}_{2}\right)$, we have

(i) $\mathcal{L}_{\ell_{1}, \ell_{2}, \wp_{1}, \wp_{2}}\left(e_{00} ; \varkappa_{1}, \varkappa_{2}, d_{1}, d_{2}\right)=1$;

(ii) $\mathcal{L}_{\ell_{1}, \ell_{2}, \wp_{1}, \wp_{2}}\left(e_{10} ; \varkappa_{1}, \varkappa_{2}, \mathrm{~d}_{1}, \mathrm{~d}_{2}\right)=\frac{1}{\ell_{1}+1}\left[\ell_{1} \varkappa_{1}+\frac{\wp_{1}+2}{\wp_{1}+1}\right]$;

(iii) $\mathcal{L}_{\ell_{1}, \ell_{2}, \wp_{1}, \wp_{2}}\left(e_{01} ; \varkappa_{1}, \varkappa_{2}, d_{1}, d_{2}\right)=\frac{1}{\ell_{2}+1}\left[\ell_{2} \varkappa_{2}+\frac{\wp_{2}+2}{\wp_{2}+1}\right]$;

(iv) $\mathcal{L}_{\ell_{1}, \ell_{2}, \wp_{1}, \wp_{2}}\left(e_{20} ; \varkappa_{1}, \varkappa_{2}, \mathrm{~d}_{1}, \mathrm{~d}_{2}\right)=\frac{1}{\left(\ell_{1}+1\right)^{2}}\left[\ell_{1}^{2} \varkappa_{1}^{2}+\ell_{1} \varkappa_{1}\left(3+\frac{1}{\mathrm{~d}_{1}-1}+\frac{2}{\wp_{1}+1}\right)+\frac{2}{\wp_{1}+1}+\frac{1}{2 \wp_{1}+1}+2\right]$;

(v) $\mathcal{L}_{\ell_{1}, \ell_{2}, \wp_{1}, \wp_{2}}\left(e_{02} ; \varkappa_{1}, \varkappa_{2}, \mathrm{~d}_{1}, \mathrm{~d}_{2}\right)=\frac{1}{\left(\ell_{2}+1\right)^{2}}\left[\ell_{2}^{2} \varkappa_{2}^{2}+\ell_{2} \varkappa_{2}\left(3+\frac{1}{\mathrm{~d}_{2}-1}+\frac{2}{\wp_{2}+1}\right)+\frac{2}{\wp_{2}+1}+\frac{1}{2 \wp_{2}+1}+2\right]$;

(vi) $\mathcal{L}_{\ell_{1}, \ell_{2}, \wp_{1}, \wp_{2}}\left(e_{30} ; \varkappa_{1}, \varkappa_{2}, d_{1}, d_{2}\right)=\frac{1}{\left(\ell_{1}+1\right)^{3}}\left[\ell_{1}^{3} \varkappa_{1}^{3}+\ell_{1}^{2} \varkappa_{1}^{2}\left(6+\frac{3}{d_{1}-1}+\frac{3}{\wp_{1}+1}\right)\right.$ $\left.+\ell_{1} \varkappa_{1}\left\{\frac{3}{2 \wp_{1}+1}+\frac{3}{\wp_{1}+1}\left(3+\frac{1}{d_{1}-1}\right)+2\left(\frac{1}{\left(d_{1}-1\right)^{2}}+\frac{3}{d_{1}-1}+5\right)\right\}+\frac{6}{\wp_{1}+1}+\frac{1}{3 \wp_{1}+1}+\frac{3}{2 \wp_{1}+1}+5\right]$

(vii) $\mathcal{L}_{\ell_{1}, \ell_{2}, \wp_{1}, \wp_{2}}\left(e_{03} ; \varkappa_{1}, \varkappa_{2}, \mathrm{~d}_{1}, \mathrm{~d}_{2}\right)=\frac{1}{\left(\ell_{2}+2\right)^{3}}\left[\ell_{2}^{3} \varkappa_{2}^{3}+\ell_{2}^{2} \varkappa_{2}^{2}\left(6+\frac{3}{\mathrm{~d}_{2}-1}+\frac{3}{\wp_{2}+1}\right)\right.$ $\left.+\ell_{2} \varkappa_{2}\left\{\frac{3}{2 \wp_{2}+1}+\frac{3}{\wp_{2}+1}\left(3+\frac{1}{d_{2}-1}\right)+2\left(\frac{1}{\left(d_{2}-1\right)^{2}}+\frac{3}{d_{2}-1}+5\right)\right\}+\frac{6}{\wp_{2}+1}+\frac{1}{3 \wp_{2}+1}+\frac{3}{2 \wp_{2}+1}+5\right]$;

(viii) $\mathcal{L}_{\ell_{1}, \ell_{2}, \wp_{1}, \wp_{2}}\left(e_{40} ; \varkappa_{1}, \varkappa_{2}, \mathrm{~d}_{1}, \mathrm{~d}_{2}\right)=\frac{1}{\left(\ell_{1}+1\right)^{4}}\left[\ell_{1}^{4} \varkappa_{1}^{4}+\ell_{1}^{3} \varkappa_{1}^{3}\left(\frac{6}{\mathrm{~d}_{1}-1}+\frac{4}{\wp_{1}+1}+10\right)\right.$ $+\ell_{1}^{2} \varkappa_{1}^{2}\left\{\frac{4}{\wp_{1}+1}\left(6+\frac{3}{d_{1}-1}\right)+\frac{30}{d_{1}-1}+\frac{11}{\left(d_{1}-1\right)^{2}}+\frac{6}{2 \wp_{1}+1}+31\right\}+\ell_{1} \varkappa_{1}\left\{\frac{6}{2 \wp_{1}+1}\left(3+\frac{1}{d_{1}-1}\right)\right.$ 


$$
\begin{aligned}
& \left.+\frac{4}{\wp_{1}+1}\left(10+\frac{2}{d_{1}-1}\left(3+\frac{1}{d_{1}-1}\right)\right)+\frac{31}{d_{1}-1}+\frac{20}{\left(d_{1}-1\right)^{2}}+\frac{6}{\left(d_{1}-1\right)^{3}}+\frac{4}{3 \wp_{1}+1}+37\right\}+\frac{1}{4 \wp_{1}+1}+\frac{4}{3 \wp_{1}+1}+\frac{12}{2 \wp_{1}+1} \\
& \left.+\frac{20}{2 \wp_{1}+1}+15\right]
\end{aligned}
$$

(ix) $\mathcal{L}_{\ell_{1}, \ell_{2}, \wp_{1}, \wp_{2}}\left(e_{04} ; \varkappa_{1}, \varkappa_{2}, \mathrm{~d}_{1}, \mathrm{~d}_{2}\right)=\frac{1}{\left(\ell_{2}+1\right)^{4}}\left[\ell_{2}^{4} \varkappa_{2}^{4}+\ell_{2}^{3} \varkappa_{2}^{3}\left(\frac{6}{\mathrm{~d}_{2}-1}+\frac{4}{\wp_{2}+1}+10\right)\right.$

$$
\begin{aligned}
& +\ell_{2}^{2} \varkappa_{2}^{2}\left\{\frac{4}{\wp_{2}+1}\left(6+\frac{3}{\mathrm{~d}_{2}-1}\right)+\frac{30}{\mathrm{~d}_{2}-1}+\frac{11}{\left(\mathrm{~d}_{2}-1\right)^{2}}+\frac{6}{2 \wp_{2}+1}+31\right\}+\ell_{2} \varkappa_{2}\left\{\frac{6}{2 \wp_{2}+1}\left(3+\frac{1}{\mathrm{~d}_{2}-1}\right)\right. \\
& \left.+\frac{4}{\wp_{2}+1}\left(10+\frac{2}{\mathrm{~d}_{2}-1}\left(3+\frac{1}{\mathrm{~d}_{2}-1}\right)\right)+\frac{31}{\mathrm{~d}_{2}-1}+\frac{20}{\left(\mathrm{~d}_{2}-1\right)^{2}}+\frac{6}{\left(\mathrm{~d}_{2}-1\right)^{3}}+\frac{4}{3 \wp_{2}+1}+37\right\}+\frac{1}{4 \wp_{2}+1}+\frac{4}{3 \wp_{2}+1}+\frac{12}{2 \wp_{2}+1} \\
& \left.+\frac{20}{2 \wp_{2}+1}+15\right] .
\end{aligned}
$$

As a consequence of the above Lemma, we have the following.

Lemma 4.2. For the operators $\mathcal{L}_{\ell_{1}, \ell_{2}, \wp_{1}, \wp_{2}}$ defined by (4.1), we have

(i) $\mathcal{L}_{\ell_{1}, \ell_{2}, \wp_{1}, \wp_{2}}\left(\left(s_{1}-\varkappa_{1}\right) ; \varkappa_{1}, \varkappa_{2}, \mathrm{~d}_{1}, \mathrm{~d}_{2}\right)=\frac{1}{\ell_{1}+1}\left[-\varkappa_{1}+\frac{\wp_{1}+2}{\wp_{1}+1}\right]$;

(ii) $\mathcal{L}_{\ell_{1}, \ell_{2}, \wp_{1}, \wp_{2}}\left(\left(s_{2}-\varkappa_{2}\right) ; \varkappa_{1}, \varkappa_{2}, \mathrm{~d}_{1}, \mathrm{~d}_{2}\right)=\frac{1}{\ell_{2}+1}\left[-\varkappa_{2}+\frac{\wp_{2}+2}{\wp_{2}+1}\right]$;

(iii) $\mathcal{L}_{\ell_{1}, \ell_{2}, \wp_{1}, \wp_{2}}\left(\left(s_{1}-\varkappa_{1}\right)^{2} ; \varkappa_{1}, \varkappa_{2}, d_{1}, d_{2}\right)=\frac{1}{\left(\ell_{1}+1\right)^{2}}\left[x_{1}^{2}+\varkappa_{1}\left\{\ell_{1}\left(3+\frac{1}{d_{1}-1}+\frac{2}{\wp_{1}+1}\right)-2\left(\ell_{1}+1\right) \frac{\wp_{1}+2}{\wp_{1}+1}\right\}\right.$ $\left.+\frac{4 \wp_{1}^{2}+11 \wp_{1}+5}{\left(\wp_{1}+1\right)\left(2 \wp_{1}+1\right)}\right]$

(iv) $\mathcal{L}_{\ell_{1}, \ell_{2}, \wp_{1}, \wp_{2}}\left(\left(s_{2}-\varkappa_{2}\right)^{2} ; \varkappa_{1}, \varkappa_{2}, \varkappa_{1}, \varkappa_{2}\right)=\frac{1}{\left(\ell_{2}+1\right)^{2}}\left[\varkappa_{2}^{2}+\varkappa_{2}\left\{\ell_{2}\left(3+\frac{1}{\mathrm{~d}_{2}-1}+\frac{2}{\wp_{2}+1}\right)-2\left(\ell_{2}+1\right) \frac{\wp_{2}+2}{\wp_{2}+1}\right\}\right.$ $\left.+\frac{4 \wp_{2}^{2}+11 \wp_{2}+5}{\left(\wp_{2}+1\right)\left(2 \wp_{2}+1\right)}\right]$

(v) $\mathcal{L}_{\ell_{1}, \ell_{2}, \wp_{1}, \wp_{2}}\left(\left(s_{1}-\varkappa_{1}\right)^{4} ; \varkappa_{1}, \varkappa_{2}, d_{1}, d_{2}\right)=\frac{1}{\left(\ell_{1}+1\right)^{4}}\left[\varkappa_{1}^{4}+\varkappa_{1}^{3}\left\{\ell_{1}^{3}\left(\frac{4}{\wp_{1}+1}+2\left(5+\frac{1}{d_{1}-1}\right)\right)\right.\right.$ $\left.+6 \ell_{1}\left(\ell_{1}+1\right)^{2}\left(\frac{2}{\wp_{1}+1}+\left(3+\frac{1}{\mathrm{~d}_{1}+1}\right)\right)-4 \ell_{1}^{2}\left(\ell_{1}+1\right)\left(\frac{3}{\wp_{1}+1}+\left(6+\frac{3}{\mathrm{~d}_{1}-1}\right)\right)-4 \ell_{1}\left(\ell_{1}+1\right)^{3}\right\}$ $+\varkappa_{1}^{2}\left\{\ell_{1}^{2}\left(\frac{6}{2 \wp_{1}+1}+4\left(6+\frac{1}{d_{1}-1}\right)+\frac{1}{d_{1}-1}\left(30+\frac{11}{d_{1}-1}\right)+31\right)+6 \ell_{1}\left(\ell_{1}+1\right)^{2}\left(\frac{1}{2 \wp_{1}+1}+2\left(1+\frac{1}{\wp_{1}+1}\right)\right)\right\}$ $\left.-4 \ell_{1}\left(\ell_{1}+1\right)\left(\frac{3}{2 \wp_{1}+1}+\frac{3}{\wp_{1}+1}\left(3+\frac{1}{d_{1}-1}\right)+2\left(5+\frac{1}{d_{1}-1}\left(3+\frac{1}{d_{1}-1}\right)\right)\right)\right\}+\varkappa_{1}\left\{\ell_{1}\left(\frac{4}{3 \wp_{1}+1}\right.\right.$ $\left.+\frac{6}{2 \wp_{1}+1}\left(3+\frac{1}{d_{1}-1}\right)+\frac{4}{\wp_{1}+1}\left(10+\frac{2}{d_{1}-1}\left(3+\frac{1}{d_{1}-1}\right)\right)+\frac{1}{d_{1}-1}\left(31+\frac{1}{d_{1}-1}\left(20+\frac{6}{d_{1}-1}\right)\right)+37\right)$ $\left.\left.-4 \ell_{1}\left(\ell_{1}+1\right)\left(\frac{1}{3 \wp_{1}+1}+\frac{3}{2 \wp_{1}+1}+\frac{6}{\wp_{1}+1}+5\right)\right\}+\frac{1}{4 \wp_{1}+1}+\frac{4}{3 \wp_{1}+1}+\frac{12}{2 \wp_{1}+1}+\frac{20}{\wp_{1}+1}+15\right]$;

(vi) $\mathcal{L}_{\ell_{1}, \ell_{2}, \wp_{1}, \wp_{2}}\left(\left(s_{2}-\varkappa_{2}\right)^{4} ; \varkappa_{1}, \varkappa_{2}, d_{1}, d_{2}\right)=\frac{1}{\left(\ell_{2}+1\right)^{4}}\left[\varkappa_{2}^{4}+\varkappa_{2}^{3}\left\{\ell_{2}^{3}\left(\frac{4}{\wp_{2}+1}+2\left(5+\frac{1}{d_{2}-1}\right)\right)\right.\right.$ $\left.+6 \ell_{2}\left(\ell_{2}+1\right)^{2}\left(\frac{2}{\wp_{2}+1}+\left(3+\frac{1}{\mathrm{~d}_{2}+1}\right)\right)-4 \ell_{2}^{2}\left(\ell_{2}+1\right)\left(\frac{3}{\wp_{2}+1}+\left(6+\frac{3}{\mathrm{~d}_{2}-1}\right)\right)-4 \ell_{2}\left(\ell_{2}+1\right)^{3}\right\}$ $+\varkappa_{2}^{2}\left\{\ell_{2}^{2}\left(\frac{6}{2 \wp_{2}+1}+4\left(6+\frac{1}{\mathrm{~d}_{2}-1}\right)+\frac{1}{\mathrm{~d}_{2}-1}\left(30+\frac{11}{\mathrm{~d}_{2}-1}\right)+31\right)+6 \ell_{2}\left(\ell_{2}+1\right)^{2}\left(\frac{1}{2 \mathfrak{\wp}_{2}+1}+2\left(1+\frac{1}{\wp_{2}+1}\right)\right)\right\}$ $\left.-4 \ell_{2}\left(\ell_{2}+1\right)\left(\frac{3}{2 \wp_{2}+1}+\frac{3}{\wp_{2}+1}\left(3+\frac{1}{d_{2}-1}\right)+2\left(5+\frac{1}{d_{2}-1}\left(3+\frac{1}{d_{2}-1}\right)\right)\right)\right\}+\varkappa_{2}\left\{m_{2}\left(\frac{4}{3 \wp_{2}+1}\right.\right.$ 


$$
\begin{aligned}
& \left.+\frac{6}{2 \wp_{2}+1}\left(3+\frac{1}{\mathrm{~d}_{2}-1}\right)+\frac{4}{\wp_{2}+1}\left(10+\frac{2}{\mathrm{~d}_{2}-1}\left(3+\frac{1}{\mathrm{~d}_{2}-1}\right)\right)+\frac{1}{\mathrm{~d}_{2}-1}\left(31+\frac{1}{\mathrm{~d}_{2}-1}\left(20+\frac{6}{\mathrm{~d}_{2}-1}\right)\right)+37\right) \\
& \left.\left.-4 \ell_{2}\left(\ell_{2}+1\right)\left(\frac{1}{3 \wp_{2}+1}+\frac{3}{2 \wp_{2}+1}+\frac{6}{\wp_{2}+1}+5\right)\right\}+\frac{1}{4 \wp_{2}+1}+\frac{4}{3 \wp_{2}+1}+\frac{12}{2 \wp_{2}+1}+\frac{20}{\wp_{2}+1}+15\right] .
\end{aligned}
$$

Remark 4.3. From Lemma 4.2, we have

(i) $\lim _{\ell_{1} \rightarrow \infty} \ell_{1} \mathcal{L}_{\ell_{1}, \ell_{2}, \wp_{1}, \wp_{2}}\left(\left(s_{1}-\varkappa_{1}\right) ; \varkappa_{1}, \varkappa_{2}, \mathrm{~d}_{1}, \mathrm{~d}_{2}\right)=-\varkappa_{1}+\frac{\wp_{1}+2}{\wp_{1}+1}$;

(ii) $\lim _{\ell_{2} \rightarrow \infty} \ell_{2} \mathcal{L}_{\ell_{1}, \ell_{2}, \wp_{1}, \wp_{2}}\left(\left(s_{2}-\varkappa_{2}\right) ; \ell_{1}, \ell_{2}, d_{1}, d_{2}\right)=-\ell_{2}+\frac{\wp_{2}+2}{\wp_{2}+1}$;

(iii) $\lim _{\ell_{1}, \ell_{2} \rightarrow \infty} \ell_{1} \ell_{2} \mathcal{L}_{\ell_{1}, \ell_{2}, \wp_{1}, \wp_{2}}\left(\left(s_{1}-\varkappa_{1}\right)\left(s_{2}-\ell_{2}\right) ; \ell_{1}, \ell_{2}, d_{1}, d_{2}\right)=0$;

(iv) $\lim _{\ell_{1} \rightarrow \infty} \ell_{1} \mathcal{L}_{\ell_{1}, \ell_{2}, \wp_{1}, \wp_{2}}\left(\left(s_{1}-\varkappa_{1}\right)^{2} ; \varkappa_{1}, \varkappa_{2}, \mathrm{~d}_{1}, \mathrm{~d}_{2}\right)=\frac{\mathrm{d}_{1} \varkappa_{1}}{\mathrm{~d}_{1}-1}$;

(v) $\lim _{\ell_{2} \rightarrow \infty} \ell_{2} \mathcal{L}_{\ell_{1}, \ell_{2}, \wp_{1}, \wp_{2}}\left(\left(s_{2}-\varkappa_{2}\right)^{2} ; \varkappa_{1}, \varkappa_{2}, \mathrm{~d}_{1}, \mathrm{~d}_{2}\right)=\frac{\mathrm{d}_{2} \varkappa_{2}}{\mathrm{~d}_{2}-1}$;

(vi) $\lim _{\ell_{1} \rightarrow \infty} \ell_{1}^{2} \mathcal{L}_{\ell_{1}, \ell_{2}, \wp_{1}, \wp_{2}}\left(\left(s_{1}-\varkappa_{1}\right)^{4} ; \varkappa_{1}, \varkappa_{2}, d_{1}, d_{2}\right)=\varkappa_{1}^{2}\left(\frac{36}{\wp_{1}+1}+\frac{12}{\left(d_{1}-1\right)\left(\wp_{1}+1\right)}+\frac{13}{\left(d_{1}-1\right)^{2}}+\frac{32}{d_{1}-1}+41\right)$;

(vii) $\lim _{\ell_{2} \rightarrow \infty} \ell_{2}^{2} \mathcal{L}_{\ell_{1}, \ell_{2}, \wp_{1}, \wp_{2}}\left(\left(s_{2}-\varkappa_{2}\right)^{4} ; \varkappa_{1}, \varkappa_{2}, \mathrm{~d}_{1}, \mathrm{~d}_{2}\right)=\varkappa_{2}^{2}\left(\frac{36}{\wp_{2}+1}+\frac{12}{\left(\mathrm{~d}_{2}-1\right)\left(\wp_{2}+1\right)}+\frac{13}{\left(\mathrm{~d}_{2}-1\right)^{2}}+\frac{32}{\mathrm{~d}_{2}-1}+41\right)$.

In what follows, we assume

$$
\mu_{\ell_{1}, \wp_{1}, d_{1}, i}\left(\varkappa_{1}\right)=\mathcal{L}_{\ell_{1}, \ell_{2}, \wp_{1}, \wp_{2}}\left(\left(s_{1}-\varkappa_{1}\right)^{i} ; \varkappa_{1}, \varkappa_{2}, d_{1}, d_{2}\right)
$$

and

$$
\mu_{\ell_{2}, \wp_{2}, d_{2}, i}\left(\varkappa_{2}\right)=\mathcal{L}_{\ell_{1}, \ell_{2}, \wp_{1}, \wp_{2}}\left(\left(s_{2}-\varkappa_{2}\right)^{i} ; \varkappa_{1}, \varkappa_{2}, d_{1}, d_{2}\right), i \in \mathbb{N} \cup\{0\} .
$$

Theorem 4.4. For any $\varphi \in \mathrm{C}_{\gamma_{1}, \gamma_{2}}\left(\mathbb{R}_{+}^{2}\right)$, we have

$$
\lim _{\ell_{1}, \ell_{2} \rightarrow \infty} \mathcal{L}_{\ell_{1}, \ell_{2}, \wp_{1}, \wp_{2}}\left(\varphi ; \varkappa_{1}, \varkappa_{2}, \mathrm{~d}_{1}, \mathrm{~d}_{2}\right)=\varphi\left(\varkappa_{1}, \varkappa_{2}\right),
$$

convergence holds uniformly on every compact subset of $\mathbb{R}_{+}^{2}$.

Proof. From Lemma 4.1, we have

$$
\lim _{\ell_{1}, \ell_{2} \rightarrow \infty} \mathcal{L}_{\ell_{1}, \ell_{2}, \wp_{1}, \wp_{2}}\left(e_{i j} ; \varkappa_{1}, \varkappa_{2}, d_{1}, d_{2}\right)=e_{i j}, i, j \in\{0,1,2,3,4\} \text { with } i+j \leqslant 4,
$$

uniformly on every compact subset of $\mathbb{R}_{+}^{2}$. The result follows applying Theorem 2.1 of [5].

Next, the degree of approximation of the operators $\mathcal{L}_{\ell_{1}, \ell_{2}, \alpha_{1}, \alpha_{2}}\left(. ; \varkappa_{1}, \varkappa_{2}, d_{1}, d_{2}\right)$ will be established in the space of continuous functions on $\mathbb{R}_{+}^{2}$. Let $C_{B}\left(\mathbb{R}_{+}^{2}\right):=\left\{\varphi \in C\left(\mathbb{R}_{+}^{2}\right):\left|\varphi\left(\varkappa_{1}, \varkappa_{2}\right)\right| \leqslant M\right.$, for some $M>0$ and all $\left.\left(\varkappa_{1}, \varkappa_{2}\right) \in \mathbb{R}_{+}^{2}\right\}$. Further, let $\overline{\mathrm{C}}_{\mathrm{B}}\left(\mathbb{R}_{+}^{2}\right):=\left\{\varphi \in \mathrm{C}_{\mathrm{B}}\left(\mathbb{R}_{+}^{2}\right): \varphi\right.$ is uniformly continuous on $\left.\mathbb{R}_{+}^{2}\right\}$. For $\varphi \in \overline{\mathrm{C}}_{\mathrm{B}}\left(\mathbb{R}_{+}^{2}\right)$ and $\delta>0$ the complete modulus of continuity for the bivariate case is defined as follows:

$$
\bar{\omega}(\varphi ; \delta)=\sup \left\{\left|\varphi\left(s_{1}, s_{2}\right)-\varphi\left(\varkappa_{1}, \varkappa_{2}\right)\right|:\left(s_{1}, s_{2}\right),\left(\varkappa_{1}, \varkappa_{2}\right) \in \mathbb{R}_{+}^{2} \text { and } \sqrt{\left(s_{1}-\varkappa_{1}\right)^{2}+\left(s_{2}-\varkappa_{2}\right)^{2}} \leqslant \delta\right\} .
$$

Further, the partial moduli of continuity with respect to $\varkappa_{1}$ and $\varkappa_{2}$ is defined as

$$
\omega_{1}(\varphi, \delta)=\sup \left\{\left|\varphi\left(z_{1}, \varkappa_{2}\right)-\varphi\left(z_{2}, \varkappa_{2}\right)\right|: \varkappa_{2} \in \mathbb{R}_{+} \text {and }\left|z_{1}-z_{2}\right| \leqslant \delta\right\},
$$

and

$$
\omega_{2}(\varphi, \delta)=\sup \left\{\left|\varphi\left(\varkappa_{1}, z_{1}\right)-\varphi\left(\varkappa_{1}, z_{2}\right)\right|: \varkappa_{1} \in \mathbb{R}_{+} \text {and }\left|z_{1}-z_{2}\right| \leqslant \delta\right\} .
$$

It is clear that they satisfy the properties of the usual modulus of continuity. The details of the modulus of continuity for the bivariate case can be found in [9]. Now, we give the estimate of the rate of convergence of the bivariate operators defined by (4.1) in terms of the complete modulus of continuity. 
Theorem 4.5. Let $\varphi \in \overline{\mathrm{C}}_{\mathrm{B}}\left(\mathbb{R}_{+}^{2}\right)$, then, for all $\left(\varkappa_{1}, \varkappa_{2}\right) \in \mathbb{R}_{+}^{2}$, we have

$$
\left|\mathcal{L}_{\ell_{1}, \ell_{2}, \wp_{1}, \wp_{2}}\left(\varphi ; \varkappa_{1}, \varkappa_{2}, d_{1}, d_{2}\right)-\varphi\left(\varkappa_{1}, \varkappa_{2}\right)\right| \leqslant 2 \bar{\omega}\left(\varphi ; \delta_{\ell_{1}, \ell_{2}, \wp_{1}, \wp_{2}}\left(\varkappa_{1}, \varkappa_{2}\right)\right),
$$

where $\delta_{\ell_{1}, \ell_{2}, \wp_{1}, \wp_{2}}\left(\varkappa_{1}, \varkappa_{2}\right)=\left(\mu_{\ell_{1}, \wp_{1}, \mathrm{~d}_{1}, 2}\left(\varkappa_{1}\right)+\mu_{\ell_{2}, \wp_{2}, \mathrm{~d}_{2}, 2}\left(\varkappa_{2}\right)\right)^{\frac{1}{2}}$.

Proof. From the definition of the complete modulus of continuity of $\varphi$, we can write

$$
\begin{aligned}
\mid \mathcal{L}_{\ell_{1}, \ell_{2}, \wp_{1}, \wp_{2}} & \left(\varphi ; \varkappa_{1}, \varkappa_{2}, \mathrm{~d}_{1}, \mathrm{~d}_{2}\right)-\varphi\left(\varkappa_{1}, \varkappa_{2}\right) \mid \\
& \leqslant \mathcal{L}_{\ell_{1}, \ell_{2}, \wp_{1}, \wp_{2}}\left(\left|\varphi\left(\mathrm{s}_{1}, \mathrm{~s}_{2}\right)-\varphi\left(\varkappa_{1}, \varkappa_{2}\right)\right| ; \varkappa_{1}, \varkappa_{2}, \mathrm{~d}_{1}, \mathrm{~d}_{2}\right) \\
& \leqslant \mathcal{L}_{\ell_{1}, \ell_{2}, \wp_{1}, \wp_{2}}\left(\omega\left(\mathrm{g} ; \sqrt{\left(\mathrm{s}_{1}-\varkappa_{1}\right)^{2}+\left(\mathrm{s}_{2}-\varkappa_{2}\right)^{2}}\right) ; \varkappa_{1}, \varkappa_{2}, \mathrm{~d}_{1}, \mathrm{~d}_{2}\right) \\
& \leqslant \bar{\omega}(\varphi, \delta)\left[1+\frac{1}{\delta} \mathcal{L}_{\ell_{1}, \ell_{2}, \wp_{1}, \wp_{2}}\left(\sqrt{\left(\mathrm{s}_{1}-\varkappa_{1}\right)^{2}+\left(\mathrm{s}_{2}-\varkappa_{2}\right)^{2}} ; \varkappa_{1}, \varkappa_{2}, \mathrm{~b}_{1}, \mathrm{~b}_{2}\right)\right],
\end{aligned}
$$

for any $\delta>0$. Now, applying Cauchy-Schwarz inequality and using Lemma 4.1, we get

$$
\begin{aligned}
&\left|\mathcal{L}_{\left.\ell_{1}, \ell_{2}, \wp_{1}, \wp_{2}\right)}\left(\varphi ; \varkappa_{1}, \varkappa_{2}, \mathrm{~d}_{1}, \mathrm{~d}_{2}\right)-\varphi\left(\varkappa_{1}, \varkappa_{2}\right)\right| \\
& \leqslant\left.\bar{\omega}(\varphi, \delta)\left[1+\frac{1}{\delta}\left\{\mathcal{L}_{\ell_{1}, \ell_{2}, \wp_{1}, \wp_{2}}\left(\left(\mathrm{~s}_{1}-\varkappa_{1}\right)^{2}+\left(\mathrm{s}_{2}-\varkappa_{2}\right)^{2}\right) ; \varkappa_{1}, \varkappa_{2}, \mathrm{~d}_{1}, \mathrm{~d}_{2}\right)\right\}^{\frac{1}{2}}\right] \\
& \leqslant \bar{\omega}(\varphi, \delta)\left[1+\frac{1}{\delta}\left\{\mathcal{L}_{\ell_{1}, \ell_{2}, \wp_{1}, \wp_{2}}\left(\left(\mathrm{~s}_{1}-\varkappa_{1}\right)^{2} ; \varkappa_{1}, \varkappa_{2}, \mathrm{~d}_{1}, \mathrm{~d}_{2}\right)\right.\right. \\
&\left.\left.+\mathcal{L}_{\ell_{1}, \ell_{2}, \wp_{1}, \wp_{2}}\left(\left(\mathrm{~s}_{2}-\varkappa_{2}\right)^{2} ; \varkappa_{2}, \mathrm{~d}_{2}\right)\right\}^{\frac{1}{2}}\right] \\
& \leqslant \bar{\omega}(\varphi ; \delta)\left[1+\frac{1}{\delta}\left(\mu_{\ell_{1}, \wp_{1}, \mathrm{~d}_{1}, 2}\left(\varkappa_{1}\right)+\mu_{\ell_{2}, \wp_{2}, \mathrm{~d}_{2}, 2}\left(\varkappa_{2}\right)\right)^{\frac{1}{2}}\right] .
\end{aligned}
$$

Choosing $\delta:=\delta_{\ell_{1}, \ell_{2}, \wp_{1}, \wp_{2}}\left(\varkappa_{1}, \varkappa_{2}\right)$, we obtain the desired result.

Next, we obtain the degree of approximation of the operators $\mathcal{L}_{\ell_{1}, \ell_{2}, \wp_{1}, \wp_{2}}$ by means of the partial moduli continuity.

Theorem 4.6. For $\varphi \in \overline{\mathrm{C}}_{\mathrm{B}}\left(\mathbb{R}_{+}^{2}\right)$ and for all $\left(\varkappa_{1}, \varkappa_{2}\right) \in \mathbb{R}_{+}^{2}$, we have

$$
\left|\mathcal{L}_{\ell_{1}, \ell_{2}, \wp_{1}, \wp_{2}}\left(\varphi ; \varkappa_{1}, \varkappa_{2}, d_{1}, d_{2}\right)-\varphi\left(\varkappa_{1}, \varkappa_{2}\right)\right| \leqslant 2\left(\omega_{1}\left(g ; \sqrt{\mu_{\ell_{1}, \wp_{1}, d_{1}, 2}\left(\varkappa_{1}\right)}\right)+\omega_{2}\left(\varphi ; \sqrt{\mu_{\ell_{2}, \wp_{2}, d_{2}, 2}\left(\varkappa_{2}\right)}\right)\right) .
$$

Proof. Using the definition of partial moduli of continuity, the Cauchy-Schwarz inequality and Lemma 4.1, we have

$$
\begin{aligned}
&\left|\mathcal{L}_{\ell_{1}, \ell_{2}, \wp_{1}, \wp_{2}}\left(\varphi ; \varkappa_{1}, \varkappa_{2}, \mathrm{~d}_{1}, \mathrm{~d}_{2}\right)-\varphi\left(\varkappa_{1}, \varkappa_{2}\right)\right| \\
& \leqslant \\
& \leqslant \\
& \leqslant \mathcal{L}_{\ell_{1}, \ell_{2}, \ell_{2}, \wp_{1}, \wp_{2}, \wp_{2}}\left(\left|\varphi\left(\mathrm{s}_{1}, \mathrm{~s}_{2}\right)-\varphi\left(\mathrm{s}_{1}, \mathrm{~s}_{2}\right)-\varphi\left(\varkappa_{1}, \varkappa_{2}\right)\right| ; \varkappa_{1}, \varkappa_{2}, \mathrm{~d}_{1}, \mathrm{~d}_{2}\right) \\
& \leqslant \omega_{1}\left(\varphi ; \sqrt{\mu_{\ell_{1}, \wp_{1}, \mathrm{~d}_{1}, 2}\left(\varkappa_{1}\right)}\right)\left[\mathcal{L}_{\ell_{1}, \ell_{2}, \wp_{1}, \wp_{2}}\left(\mathrm{e}_{00} ; \mathrm{d}_{1}, \mathrm{~d}_{2}\right)+\mathcal{L}_{\left.\varkappa_{1}, \varkappa_{2}, \varkappa_{1}, \wp_{2}, \mathrm{~d}_{1}, \mathrm{~d}_{2}\right)}\left(\left|\varphi\left(\varkappa_{1}, \mathrm{~s}_{2}\right)-\varphi\left(\varkappa_{1}, \varkappa_{2}\right)\right| ; \varkappa_{1}, \varkappa_{2}, \mathrm{~d}_{1}, \mathrm{~d}_{2}\right)\right. \\
&\left.+\frac{1}{\sqrt{\mu_{\ell_{1}, \wp_{1}, \mathrm{~d}_{1}, 2}\left(\varkappa_{1}\right)}} \mathcal{L}_{\ell_{1}, \ell_{2}, \wp_{1}, \wp_{2}}\left(\left|\mathrm{~s}_{1}-\varkappa_{1}\right| ; \varkappa_{1}, \varkappa_{2}, \mathrm{~d}_{1}, \mathrm{~d}_{2}\right)\right] \\
&+\omega_{2}\left(\varphi ; \sqrt{\mu_{\ell_{2}, \wp_{2}, \mathrm{~d}_{2}, 2}\left(\varkappa_{2}\right)}\right)\left[\mathcal{L}_{\ell_{1}, \ell_{2}, \wp_{1}, \wp_{2}}\left(e_{00} ; \varkappa_{1}, \varkappa_{2}, \mathrm{~d}_{1}, \mathrm{~d}_{2}\right)\right.
\end{aligned}
$$




$$
\begin{aligned}
& \left.+\frac{1}{\sqrt{\mu_{\ell_{2}, \wp_{2}, \mathrm{~d}_{2,2}\left(\varkappa_{2}\right)}}} \mathcal{L}_{\ell_{1}, \ell_{2}, \wp_{1}, \wp_{2}}\left(\left|\mathrm{~s}_{2}-\varkappa_{2}\right| ; \varkappa_{1}, \varkappa_{2}, \mathrm{~d}_{1}, \mathrm{~d}_{2}\right)\right] \\
& \leqslant \omega_{1}\left(\varphi ; \sqrt{\mu_{\ell_{1}, \wp_{1}, \mathrm{~d}_{1}, 2}\left(\varkappa_{1}\right)}\right)\left[1+\frac{1}{\sqrt{\mu_{\ell_{1}, \wp_{1}, \mathrm{~d}_{1}, 2}\left(\varkappa_{1}\right)}}\left(\mathcal{L}_{\ell_{1}, \ell_{2}, \wp_{1}, \wp_{2}}\left(\left(\mathrm{~s}_{1}-\varkappa_{1}\right)^{2} ; \varkappa_{1}, \varkappa_{2}, \mathrm{~d}_{1}, \mathrm{~d}_{2}\right)\right)^{\frac{1}{2}}\right] \\
& +\omega_{2}\left(\varphi ; \sqrt{\mu_{\ell_{2}, \wp_{2}, \mathrm{~d}_{2}, 2}\left(\varkappa_{2}\right)}\right)\left[1+\frac{1}{\sqrt{\mu_{\ell_{2}, \wp_{2}, \mathrm{~d}_{2}, 2}\left(\varkappa_{2}\right)}}\left(\mathcal{L}_{\ell_{1}, \ell_{2}, \wp_{1}, \wp_{1}}\left(\left(\mathrm{~s}_{2}-\varkappa_{2}\right)^{2} ; \varkappa_{1}, \varkappa_{2}, \mathrm{~d}_{1}, \mathrm{~d}_{2}\right)\right)^{\frac{1}{2}}\right],
\end{aligned}
$$

from which the required result is straightforward.

Let $\mathrm{C}_{\mathrm{B}}^{2}\left(\mathbb{R}_{+}^{2}\right)$ be the space of all functions $\varphi \in \mathrm{C}_{\mathrm{B}}\left(\mathbb{R}_{+}^{2}\right)$ such that all second order partial derivatives of $\varphi$ belong to $\mathrm{C}_{\mathrm{B}}\left(\mathbb{R}_{+}^{2}\right)$. The norm on the space $\mathrm{C}_{\mathrm{B}}^{2}\left(\mathbb{R}_{+}^{2}\right)$ is given by

$$
\|\varphi\|_{C_{B}^{2}\left(\mathbb{R}_{+}^{2}\right)}=\|\varphi\|+\sum_{i=1}^{2}\left(\left\|\frac{\partial^{i} \varphi}{\partial \varkappa_{1}^{i}}\right\|+\left\|\frac{\partial^{i} \varphi}{\partial \varkappa_{2}^{i}}\right\|\right)+\left\|\frac{\partial^{2} \varphi}{\partial \varkappa_{1} \partial \varkappa_{2}}\right\| .
$$

The appropriate Peetre's K-functional of the function $\varphi \in \overline{\mathrm{C}}_{\mathrm{B}}\left(\mathbb{R}_{+}^{2}\right)$ is defined as

$$
\mathrm{K}(\varphi, \delta)=\inf _{\mathrm{f} \in \mathrm{C}_{\mathrm{B}}^{2}\left(\mathbb{R}_{+}^{2}\right)}\left\{\|\varphi-\mathrm{f}\|+\delta\|f\|_{\mathrm{C}_{\mathrm{B}}^{2}\left(\mathbb{R}_{+}^{2}\right)}\right\}, \delta>0 .
$$

From [11], it is known that

$$
K(\varphi ; \delta) \leqslant M \bar{\omega}_{2}(\varphi ; \sqrt{\delta}) \text { holds for all } \delta>0,
$$

where $\bar{\omega}_{2}(\varphi ; \sqrt{\delta})$ is the second order modulus of continuity for the bivariate case and $M$ is a constant independent of $\delta$ and $\varphi$.

Theorem 4.7. For the function $\varphi \in \overline{\mathrm{C}}_{\mathrm{B}}\left(\mathbb{R}_{+}^{2}\right)$ and $\left(\varkappa_{1}, \varkappa_{2}\right) \in \mathbb{R}_{+}^{2}$, we have

$$
\begin{aligned}
\left|\mathcal{L}_{\ell_{1}, \ell_{2}, \wp_{1}, \wp_{2}}\left(\varphi ; \varkappa_{1}, \varkappa_{2}, \mathrm{~d}_{1}, \mathrm{~d}_{2}\right)-\varphi\left(\varkappa_{1}, \varkappa_{2}\right)\right| \leqslant & M \bar{\omega}_{2}\left(\varphi ; \frac{1}{2} \sqrt{\mathcal{A}_{\ell_{1}, \ell_{2}, \wp_{1}, \wp_{2}}\left(\varkappa_{1}, \varkappa_{2}, \mathrm{~d}_{1}, \mathrm{~d}_{2}\right)}\right) \\
& +\bar{\omega}\left(\varphi ; \sqrt{\left(\mu_{\ell_{1}, \wp_{1}, \mathrm{~d}_{1}, 1}\left(\varkappa_{1}\right)\right)^{2}+\left(\mu_{\ell_{2}, \wp_{2}, \mathrm{~d}_{2}, 1}\left(\varkappa_{2}\right)\right)^{2}}\right),
\end{aligned}
$$

where

$\mathcal{A}_{\ell_{1}, \ell_{2}, \wp_{1}, \wp_{2}}\left(\varkappa_{1}, \varkappa_{2}, \mathrm{~d}_{1}, \mathrm{~d}_{2}\right)=\frac{1}{2}\left\{\left(\sqrt{\mu_{\ell_{1}, \wp_{1}, \mathrm{~d}_{1}, 2}\left(\varkappa_{1}\right)}+\sqrt{\mu_{\ell_{2}, \wp_{2}, \mathrm{~d}_{2}, 2}\left(\varkappa_{2}\right)}\right)^{2}+\left(\left|\mu_{\ell_{1}, \wp_{1}, \mathrm{~d}_{1}, 1}\left(\varkappa_{1}\right)\right|+\left|\mu_{\ell_{2}, \wp_{2}, \mathrm{~d}_{2}, 1}\left(\varkappa_{2}\right)\right|\right)^{2}\right\}$.

Proof. We consider the auxiliary operators as follows:

$$
\begin{aligned}
\overline{\mathcal{L}}_{\ell_{1}, \ell_{2}, \wp_{1}, \wp_{2}}\left(\varphi ; \varkappa_{1}, \varkappa_{2}, \mathrm{~d}_{1}, \mathrm{~d}_{2}\right)= & \mathcal{L}_{\ell_{1}, \ell_{2}, \wp_{1}, \wp_{2}}\left(\varphi ; \varkappa_{1}, \varkappa_{2}, \mathrm{~d}_{1}, \mathrm{~d}_{2}\right) \\
& -\varphi\left(\varkappa_{1}, \varkappa_{2}\right) \varphi\left(\frac{\ell_{1} \varkappa_{1}}{\ell_{1}+1}+\frac{\wp_{1}+2}{\left(\ell_{1}+1\right)\left(\wp_{1}+1\right)}, \frac{\ell_{2} \varkappa_{2}}{\ell_{2}+1}+\frac{\wp_{2}+2}{\left(\ell_{2}+1\right)\left(\wp_{2}+1\right)}\right) .
\end{aligned}
$$

In view of Lemma 4.2, we get

$$
\overline{\mathcal{L}}_{\ell_{1}, \ell_{2}, \wp_{1}, \wp_{2}}\left(\left(s_{1}-\varkappa_{1}\right) ; \varkappa_{1}, \varkappa_{2}, \mathrm{~d}_{1}, \mathrm{~d}_{2}\right)=0 \text { and } \overline{\mathcal{L}}_{\ell_{1}, \ell_{2}, \wp_{1}, \wp_{2}}\left(\left(s_{2}-\varkappa_{2}\right) ; \varkappa_{1}, \varkappa_{2}, \mathrm{~d}_{1}, \mathrm{~d}_{2}\right)=0 .
$$

Let $h \in \mathrm{C}_{\mathrm{B}}^{2}\left(\mathbb{R}_{+}^{2}\right)$ and $\left(s_{1}, s_{2}\right),\left(\varkappa_{1}, \varkappa_{2}\right) \in \mathbb{R}_{+}^{2}$. By using the Taylor's expansion, we can write

$$
h\left(s_{1}, s_{2}\right)-h\left(\varkappa_{1}, \varkappa_{2}\right)=\left(s_{1}-\varkappa_{1}\right) \frac{\partial}{\partial \varkappa_{1}} h\left(\varkappa_{1}, \varkappa_{2}\right)+\int_{\varkappa_{1}}^{s_{1}}\left(s_{1}-u\right) \frac{\partial^{2}}{\partial u^{2}} h\left(u, \varkappa_{2}\right) d u+\left(s_{2}-\varkappa_{2}\right) \frac{\partial}{\partial \varkappa_{2}} h\left(\varkappa_{1}, \varkappa_{2}\right)
$$




$$
+\int_{\varkappa_{2}}^{s_{2}}\left(s_{2}-v\right) \frac{\partial^{2}}{\partial v^{2}} h\left(\varkappa_{1}, v\right) d v+\int_{\varkappa_{1}}^{s_{1}} \int_{\varkappa_{2}}^{s_{2}} \frac{\partial^{2} h(u, v)}{\partial u \partial v} d u d v .
$$

Now, applying the operator $\overline{\mathcal{L}}_{\ell_{1}, \ell_{2}, \wp_{1}, \wp_{2}}\left(. ; \varkappa_{1}, \varkappa_{2}, \mathrm{~d}_{1}, \mathrm{~d}_{2}\right)$ on the above equation and using (4.4), we get

$$
\begin{aligned}
& \overline{\mathcal{L}}_{\ell_{1}, \ell_{2}, \wp_{1}, \wp_{2}}\left(h ; \varkappa_{1}, \varkappa_{2}, d_{1}, d_{2}\right)-h\left(\varkappa_{1}, \varkappa_{2}\right) \\
& =\overline{\mathcal{L}}_{\ell_{1}, \ell_{2}, \wp_{1}, \wp_{2}}\left(\int_{\varkappa_{1}}^{s_{1}}\left(s_{1}-u\right) \frac{\partial^{2}}{\partial u^{2}} h\left(u, \varkappa_{2}\right) d u ; \varkappa_{1}, \varkappa_{2}, d_{1}, d_{2}\right) \\
& +\overline{\mathcal{L}}_{\ell_{1}, \ell_{2}, \wp_{1}, \wp_{2}}\left(\int_{\varkappa_{2}}^{s_{2}}\left(s_{2}-v\right) \frac{\partial^{2}}{\partial v^{2}} h\left(\varkappa_{1}, v\right) d v ; \varkappa_{1}, \varkappa_{2}, d_{1}, d_{2}\right) \\
& +\overline{\mathcal{L}}_{\ell_{1}, \ell_{2}, \wp_{1}, \wp_{2}}\left(\int_{\varkappa_{1}}^{s_{1}} \int_{\varkappa_{2}}^{s_{2}} \frac{\partial^{2} h(u, v)}{\partial u \partial v} d u d v ; \varkappa_{1}, \varkappa_{2}, d_{1}, d_{2}\right) \\
& =\mathcal{L}_{\ell_{1}, \ell_{2}, \wp_{1}, \wp_{2}}\left(\int_{\ell_{1}}^{s_{1}}\left(s_{1}-u\right) \frac{\partial^{2}}{\partial u^{2}} h\left(u, \varkappa_{2}\right) d u ; \varkappa_{1}, \varkappa_{2}, d_{1}, d_{2}\right) \\
& -\int_{\varkappa_{1}}^{\frac{\ell_{1} \varkappa_{1}}{\ell_{1}+1}+\frac{\wp_{1}+2}{\left(\ell_{1}+1\right)\left(\wp_{1}+1\right)}}\left(\frac{\ell_{1} \varkappa_{1}}{\ell_{1}+1}+\frac{\wp_{1}+2}{\left(\ell_{1}+1\right)\left(\wp_{1}+1\right)}-u\right) \frac{\partial^{2} h\left(u, \varkappa_{2}\right)}{\partial u^{2}} d u \\
& +\mathcal{L}_{\ell_{1}, \ell_{2}, \wp_{1}, \wp_{2}}\left(\int_{\varkappa_{2}}^{s_{2}}\left(s_{2}-v\right) \frac{\partial^{2}}{\partial v^{2}} h\left(\varkappa_{1}, v\right) \mathrm{d} v ; \varkappa_{1}, \varkappa_{2}, \mathrm{~d}_{1}, \mathrm{~d}_{2}\right) \\
& -\int_{\varkappa_{2}}^{\frac{\ell_{2} \varkappa_{2}}{\ell_{2}+1}+\frac{\wp_{2}+2}{\left(\ell_{2}+1\right)\left(\wp_{2}+1\right)}}\left(\frac{\ell_{2} \varkappa_{2}}{\ell_{2}+1}+\frac{\wp_{2}+2}{\left(\ell_{2}+1\right)\left(\wp_{2}+1\right)}-v\right) \frac{\partial^{2} h\left(\varkappa_{1}, v\right)}{\partial v^{2}} \mathrm{~d} v \\
& +\mathcal{L}_{\ell_{1}, \ell_{2}, \wp_{1}, \wp_{2}}\left(\int_{\varkappa_{1}}^{s_{1}} \int_{\varkappa_{2}}^{s_{2}} \frac{\partial^{2} h(u, v)}{\partial u \partial v} d u d v ; \varkappa_{1}, \varkappa_{2}, d_{1}, d_{2}\right) \\
& -\int_{\varkappa_{1}}^{\frac{\ell_{1} \varkappa_{1}}{\ell_{1}+1}+\frac{\wp_{1}+2}{\left(\ell_{1}+1\right)\left(\wp_{1}+1\right)}} \int_{\varkappa_{2}}^{\frac{\ell_{2} \varkappa_{2}}{\ell_{2}+1}+\frac{\wp_{2}+2}{\left(\ell_{2}+1\right)\left(\wp_{2}+1\right)}} \frac{\partial^{2} h(u, v)}{\partial u \partial v} d u d v .
\end{aligned}
$$

Using Lemma 4.1, we get

$$
\begin{aligned}
\left|\overline{\mathcal{L}}_{\ell_{1}, \ell_{2}, \wp_{1}, \wp_{2}}\left(\mathrm{~h} ; \varkappa_{1}, \varkappa_{2}, \mathrm{~d}_{1}, \mathrm{~d}_{2}\right)-\mathrm{h}\left(\varkappa_{1}, \varkappa_{2}\right)\right| \leqslant & {\left[\frac { 1 } { 2 } \left\{\mu_{\ell_{1}, \wp_{1}, \mathrm{~d}_{1}, 2}\left(\varkappa_{1}\right)+\left(\mu_{\ell_{1}, \wp_{1}, \mathrm{~d}_{1}, 1}\left(\varkappa_{1}\right)\right)^{2}+\mu_{\ell_{2}, \wp_{2}, \mathrm{~d}_{2}, 2}\left(\varkappa_{2}\right)\right.\right.} \\
& \left.+\left(\mu_{\ell_{2}, \wp_{2}, \mathrm{~d}_{2}, 1}\left(\varkappa_{2}\right)\right)^{2}\right\}+\sqrt{\mu_{\ell_{1}, \wp_{1}, \mathrm{~d}_{1}, 2}\left(\varkappa_{1}\right)} \sqrt{\mu_{\ell_{2}, \wp_{2}, \mathrm{~d}_{2}, 2}\left(\varkappa_{2}\right)} \\
& \left.+\left|\mu_{\ell_{1}, \wp_{1}, \mathrm{~d}_{1}, 1}\left(\varkappa_{1}\right)\right|\left|\mu_{\ell_{2}, \wp_{2}, \mathrm{~d}_{2}, 1}\left(\varkappa_{2}\right)\right|\right]\|\mathrm{h}\|_{\mathrm{C}_{\mathrm{B}}^{2}\left(\mathbb{R}_{+}^{2}\right)} \\
= & \mathcal{A}_{\ell_{1}, \ell_{2}, \wp_{1}, \wp_{2}}\left(\varkappa_{1}, \varkappa_{2}, \mathrm{~d}_{1}, \mathrm{~d}_{2}\right)\|\mathrm{h}\|_{\mathrm{C}_{\mathrm{B}}^{2}\left(\mathbb{R}_{+}^{2}\right)} .
\end{aligned}
$$

By using (4.3), we have

$$
\begin{aligned}
\left|\overline{\mathcal{L}}_{\ell_{1}, \ell_{2}, \wp_{1}, \wp_{2}}\left(\varphi ; \varkappa_{1}, \varkappa_{2}, \mathrm{~d}_{1}, \mathrm{~d}_{2}\right)\right| \leqslant & \left|\mathcal{L}_{\ell_{1}, \ell_{2}, \wp_{1}, \wp_{2}}\left(\varphi ; \varkappa_{1}, \varkappa_{2}, \mathrm{~d}_{1}, \mathrm{~d}_{2}\right)\right| \\
& +\left|\varphi\left(\varkappa_{1}, \varkappa_{2}\right)\right|+\left|\mathrm{f}\left(\frac{\ell_{1} \varkappa_{1}}{\ell_{1}+1}+\frac{\wp_{1}+2}{\left(\ell_{1}+1\right)\left(\wp_{1}+1\right)}, \frac{\ell_{2} \varkappa_{2}}{\ell_{2}+1}+\frac{\wp_{2}+2}{\left(\ell_{2}+1\right)\left(\wp_{2}+1\right)}\right)\right| \\
\leqslant & 3\|\varphi\| .
\end{aligned}
$$

Hence, for $\varphi \in \overline{\mathrm{C}}_{\mathrm{B}}\left(\mathbb{R}_{+}^{2}\right)$ and any $\mathrm{h} \in \mathrm{C}_{\mathrm{B}}^{2}\left(\mathbb{R}_{+}^{2}\right)$, we get

$$
\begin{aligned}
& \left|\mathcal{L}_{\ell_{1}, \ell_{2}, \wp_{1}, \wp_{2}}\left(\varphi ; \varkappa_{1}, \varkappa_{2}, \mathrm{~d}_{1}, \mathrm{~d}_{2}\right)-\varphi\left(\varkappa_{1}, \varkappa_{2}\right)\right| \\
& \leqslant \mid \overline{\mathcal{L}}_{\ell_{1}, \ell_{2}, \wp_{1}, \wp_{2}}\left(\varphi ; \varkappa_{1}, \varkappa_{2}, \mathrm{~d}_{1}, \mathrm{~d}_{2}\right)-\varphi\left(\varkappa_{1}, \varkappa_{2}\right)
\end{aligned}
$$




$$
\begin{aligned}
& +\varphi\left(\frac{\ell_{1} \varkappa_{1}}{\ell_{1}+1}+\frac{\wp_{1}+2}{\left(\ell_{1}+1\right)\left(\wp_{1}+1\right)}, \frac{\ell_{2} \varkappa_{2}}{\ell_{2}+1}+\frac{\wp_{2}+2}{\left(\ell_{2}+1\right)\left(\wp_{2}+1\right)}\right)-\varphi\left(\varkappa_{1}, \varkappa_{2}\right) \mid \\
\leqslant & \left|\overline{\mathcal{L}}_{\ell_{1}, \ell_{2}, \wp_{1}, \wp_{2}}\left(\varphi-\mathrm{h} ; \varkappa_{1}, \varkappa_{2}, \mathrm{~d}_{1}, \mathrm{~d}_{2}\right)\right|+\left|\varphi\left(\varkappa_{1}, \varkappa_{2}\right)-\mathrm{h}\left(\varkappa_{1}, \varkappa_{2}\right)\right| \\
& +\left|\overline{\mathcal{L}}_{\ell_{1}, \ell_{2}, \wp_{1}, \wp_{2}}\left(\mathrm{~h} ; \varkappa_{1}, \varkappa_{2}, \mathrm{~d}_{1}, \mathrm{~d}_{2}\right)-\mathrm{h}\left(\varkappa_{1}, \varkappa_{2}\right)\right| \\
& +\left|\varphi\left(\frac{\ell_{1} \varkappa_{1}}{\ell_{1}+1}+\frac{\wp_{1}+2}{\left(\ell_{1}+1\right)\left(\wp_{1}+1\right)}, \frac{\ell_{2} \varkappa_{2}}{\ell_{2}+1}+\frac{\wp_{2}+2}{\left(\ell_{2}+1\right)\left(\wp_{2}+1\right)}\right)-\varphi\left(\varkappa_{1}, \varkappa_{2}\right)\right| \\
\leqslant & 4\|\varphi-\mathrm{h}\|+\mathcal{A}_{\ell_{1}, \ell_{2}, \wp_{1}, \wp_{2}\left(\varkappa_{1}, \varkappa_{2}, \mathrm{~d}_{1}, \mathrm{~d}_{2}\right)\|\mathrm{h}\| \mathrm{C}_{\mathrm{B}}^{2}\left(\mathbb{R}_{+}^{2}\right)} \\
& +\bar{\omega}\left(\varphi ; \sqrt{\left(\mu_{\ell_{1}, \wp_{1}, \mathrm{~d}_{1}, 1}\left(\varkappa_{1}\right)\right)^{2}+\left(\mu_{\ell_{2}, \wp_{2}, \mathrm{~d}_{2}, 1}\left(\varkappa_{2}\right)\right)^{2}}\right) .
\end{aligned}
$$

Now, taking the infimum on the right hand side over all $\mathrm{h} \in \mathrm{C}_{\mathrm{B}}^{2}\left(\mathbb{R}_{+}^{2}\right)$ and using relation (4.2), we get $\left.\mid \mathcal{L}_{\ell_{1}, \ell_{2}, \wp_{1}, \wp_{2}}\left(\varphi ; \varkappa_{1}, \varkappa_{2}, \mathrm{~d}_{1}, \mathrm{~d}_{2}\right)-\varphi\left(\varkappa_{1}, \varkappa_{2}\right)\right) \mid$

$$
\begin{aligned}
& \leqslant 4 \mathrm{~K}\left(\varphi ; \frac{\mathcal{A}_{\ell_{1}, \ell_{2}, \wp_{1}, \wp_{2}}\left(\varkappa_{1}, \varkappa_{2}, \mathrm{~d}_{1}, \mathrm{~d}_{2}\right)}{4}\right)+\bar{\omega}\left(\varphi ; \sqrt{\left(\mu_{\ell_{1}, \wp_{1}, \mathrm{~d}_{1}, 1}\left(\varkappa_{1}\right)\right)^{2}+\left(\mu_{\ell_{2}, \wp_{2}, \mathrm{~d}_{2}, 1}\left(\varkappa_{2}\right)\right)^{2}}\right) \\
& \leqslant M \bar{\omega}_{2}\left(\varphi ; \frac{1}{2} \sqrt{\mathcal{A}_{\ell_{1}, \ell_{2}, \wp_{1}, \wp_{2}}\left(\varkappa_{1}, \varkappa_{2}, \mathrm{~d}_{1}, \mathrm{~d}_{2}\right)}\right)+\bar{\omega}\left(\varphi ; \sqrt{\left(\mu_{\ell_{1}, \wp_{1}, \mathrm{~d}_{1}, 1}\left(\varkappa_{1}\right)\right)^{2}+\left(\mu_{\ell_{2}, \wp_{2}, \mathrm{~d}_{2}, 1}\left(\varkappa_{2}\right)\right)^{2}}\right) .
\end{aligned}
$$

This completes the proof of the theorem.

Let $\mathrm{C}_{\mathrm{B}}^{1}\left(\mathbb{R}_{+}^{2}\right):=\left\{\varphi \in \mathrm{C}_{\mathrm{B}}\left(\mathbb{R}_{+}^{2}\right): \varphi_{\varkappa_{1}}^{\prime}, \varphi_{\varkappa_{2}}^{\prime} \in \mathrm{C}_{\mathrm{B}}\left(\mathbb{R}_{+}^{2}\right)\right\}$.

Theorem 4.8. For $\varphi \in \mathrm{C}_{\mathrm{B}}^{1}\left(\mathbb{R}_{+}^{2}\right)$ and $\left(\varkappa_{1}, \varkappa_{2}\right) \in \mathbb{R}_{+}^{2}$, we have

$$
\left|\mathcal{L}_{\ell_{1}, \ell_{2}, \wp_{1}, \wp_{2}}\left(\varphi ; \varkappa_{1}, \varkappa_{2}, \mathrm{~d}_{1}, \mathrm{~d}_{2}\right)-\varphi\left(\varkappa_{1}, \varkappa_{2}\right)\right| \leqslant\left\|\varphi_{\varkappa_{1}}^{\prime}\right\| \sqrt{\mu_{\ell_{1}, \wp_{1}, \mathrm{~d}_{1}, 2}\left(\varkappa_{1}\right)}+\left\|\varphi_{\varkappa_{2}}^{\prime}\right\| \sqrt{\mu_{\ell_{2}, \wp_{2}, \mathrm{~d}_{2}, 2}\left(\varkappa_{2}\right)} .
$$

Proof. Let $\left(\varkappa_{1}, \varkappa_{2}\right) \in \mathbb{R}_{+}^{2}$ be a fixed point. Then by our hypothesis, we can write

$$
\varphi\left(s_{1}, s_{2}\right)-\varphi\left(\varkappa_{1}, \varkappa_{2}\right)=\int_{\varkappa_{1}}^{s_{1}} \varphi_{w}^{\prime}\left(w, s_{2}\right) d w+\int_{\varkappa_{2}}^{s_{2}} \varphi_{u}^{\prime}\left(\varkappa_{1}, u\right) d u .
$$

Now, applying the operator $\mathcal{L}_{\ell_{1}, \ell_{2}, \wp_{1}, \wp_{2}}\left(; \varkappa_{1}, \varkappa_{2}, d_{1}, d_{2}\right)$ on both sides of the above eqaution, we are led to

$$
\begin{aligned}
\left|\mathcal{L}_{\ell_{1}, \ell_{2}, \wp_{1}, \wp_{2}}\left(\varphi ; \varkappa_{1}, \varkappa_{2}, \mathrm{~d}_{1}, \mathrm{~d}_{2}\right)-\varphi\left(\varkappa_{1}, \varkappa_{2}\right)\right| \leqslant & \mathcal{L}_{\ell_{1}, \ell_{2}, \wp_{1}, \wp_{2}}\left(\left|\int_{\varkappa_{1}}^{s_{1}}\right| \varphi_{w}^{\prime}\left(w, \mathrm{~s}_{2}\right)|\mathrm{d} w| ; \varkappa_{1}, \varkappa_{2}, \mathrm{~d}_{1}, \mathrm{~d}_{2}\right) \\
& +\mathcal{L}_{\ell_{1}, \ell_{2}, \wp_{1}, \wp_{2}}\left(\left|\int_{\varkappa_{2}}^{s_{2}}\right| \varphi_{u}^{\prime}\left(\varkappa_{1}, \mathrm{u}\right)|\mathrm{du}| ; \varkappa_{1}, \varkappa_{2}, \mathrm{~d}_{1}, \mathrm{~d}_{2}\right) .
\end{aligned}
$$

Since

$$
\left|\int_{\varkappa_{1}}^{s_{1}}\right| \varphi_{w}^{\prime}\left(w, s_{2}\right)|d w| \leqslant\left\|\varphi_{\varkappa_{1}}^{\prime}\right\|\left|s_{1}-\varkappa_{1}\right| \text { and }\left|\int_{\varkappa_{2}}^{s_{2}}\right| \varphi_{\mathfrak{u}}^{\prime}\left(\varkappa_{1}, u\right)|d u| \leqslant\left\|\varphi_{\varkappa_{2}}^{\prime}\right\|\left|s_{2}-\varkappa_{2}\right| .
$$

We have

$$
\begin{aligned}
\left|\mathcal{L}_{\left.\ell_{1}, \ell_{2}, \wp_{1}, \wp_{2}\right)}\left(\varphi ; \varkappa_{1}, \varkappa_{2}, \mathrm{~d}_{1}, \mathrm{~d}_{2}\right)-\varphi\left(\varkappa_{1}, \varkappa_{2}\right)\right| \leqslant & \left\|\varphi_{\varkappa_{1}}^{\prime}\right\| \mathcal{L}_{\ell_{1}, \ell_{2}, \wp_{1}, \wp_{2}}\left(\left|s_{1}-\varkappa_{1}\right| ; \varkappa_{1}, \varkappa_{2}, \mathrm{~d}_{1}, \mathrm{~d}_{2}\right) \\
& +\left\|\varphi_{\varkappa_{2}}^{\prime}\right\| \mathcal{L}_{\ell_{1}, \ell_{2}, \wp_{1}, \wp_{2}}\left(\left|s_{2}-\varkappa_{2}\right| ; \varkappa_{1}, \varkappa_{2}, \mathrm{~d}_{1}, \mathrm{~d}_{2}\right) .
\end{aligned}
$$

Now, using Cauchy-Schwarz inequality and Lemma 4.1, we get

$$
\begin{aligned}
& \left|\mathcal{L}_{\ell_{1}, \ell_{2}, \wp_{1}, \wp_{2}}\left(\varphi ; \varkappa_{1}, \varkappa_{2}, \mathrm{~d}_{1}, \mathrm{~d}_{2}\right)-\varphi\left(\varkappa_{1}, \varkappa_{2}\right)\right| \\
& \quad \leqslant\left\|\varphi_{\varkappa_{1}}^{\prime}\right\|\left(\mathcal{L}_{\ell_{1}, \ell_{2}, \wp_{1}, \wp_{2}}\left(\left(\mathrm{~s}_{1}-\varkappa_{1}\right)^{2} ; \varkappa_{1}, \varkappa_{2}, \mathrm{~d}_{1}, \mathrm{~d}_{2}\right)\right)^{\frac{1}{2}}\left(\mathcal{L}_{\ell_{1}, \ell_{2}, \wp_{1}, \wp_{2}}\left(e_{00} ; \varkappa_{1}, \varkappa_{2}, \mathrm{~d}_{1}, \mathrm{~d}_{2}\right)\right)^{\frac{1}{2}}
\end{aligned}
$$




$$
\begin{aligned}
& +\left\|\varphi_{\varkappa_{2}}^{\prime}\right\|\left(\mathcal{L}_{\ell_{1}, \ell_{2}, \wp_{1}, \wp_{2}}\left(\left(s_{2}-\ell_{2}\right)^{2} ; \ell_{1}, \ell_{2}, \mathrm{~d}_{1}, \mathrm{~d}_{2}\right)\right)^{\frac{1}{2}}\left(\mathcal{L}_{\ell_{1}, \ell_{2}, \wp_{1}, \wp_{2}}\left(e_{00} ; \varkappa_{1}, \varkappa_{2}, \mathrm{~d}_{1}, \mathrm{~d}_{2}\right)\right)^{\frac{1}{2}} \\
& \leqslant\left\|\varphi_{\varkappa_{1}}^{\prime}\right\| \sqrt{\mu_{\ell_{1}, \wp_{1}, \mathrm{~d}_{2}, 2}\left(\varkappa_{1}\right)}+\left\|\varphi_{\varkappa_{2}}^{\prime}\right\| \sqrt{\mu_{\ell_{2}, \wp_{2}, \mathrm{~d}_{2}, 2}\left(\varkappa_{2}\right)} .
\end{aligned}
$$

This completes the proof.

Theorem 4.9. Let $\varphi^{\prime \prime} \in \mathrm{C}_{\gamma_{1}, \gamma_{2}}\left(\mathbb{R}_{+}^{2}\right)$, for some $\gamma_{1}, \gamma_{2}>0$. Then,

$$
\begin{aligned}
\lim _{\ell \rightarrow \infty} \ell\left(\mathcal{L}_{\ell, \ell, \wp_{1}, \wp_{2}}\left(\varphi ; \varkappa_{1}, \varkappa_{2}, \mathrm{~d}_{1}, \mathrm{~d}_{2}\right)-\varphi\left(\varkappa_{1}, \varkappa_{2}\right)\right)= & g_{\varkappa_{1}}^{\prime}\left(\varkappa_{1}, \varkappa_{2}\right)\left(-\varkappa_{1}+\frac{\wp_{1}+2}{\wp_{1}+1}\right)+\varphi_{\varkappa_{2}}^{\prime}\left(\varkappa_{1}, \varkappa_{2}\right)\left(-\varkappa_{2}+\frac{\wp_{2}+2}{\wp_{2}+1}\right) \\
& +\frac{1}{2}\left\{\frac{\mathrm{d}_{1} \varkappa_{1}}{\mathrm{~d}_{1}-1} \varphi_{\varkappa_{1} \varkappa_{1}}^{\prime \prime}\left(\varkappa_{1}, \varkappa_{2}\right)+\frac{\mathrm{d}_{2} \varkappa_{2}}{\mathrm{~d}_{2}-1} \varphi_{\varkappa_{2} \varkappa_{2}}^{\prime \prime}\left(\varkappa_{1}, \varkappa_{2}\right)\right\},
\end{aligned}
$$

uniformly on each compact subset of $\mathbb{R}_{+}^{2}$.

Proof. By the Taylor's expansion, we have

$$
\begin{aligned}
\varphi\left(s_{1}, s_{2}\right)= & \varphi\left(\varkappa_{1}, \varkappa_{2}\right)+\left(s_{1}-\varkappa_{1}\right) \varphi_{\varkappa_{1}}^{\prime}\left(\varkappa_{1}, \varkappa_{2}\right)+\left(s_{2}-\varkappa_{2}\right) \varphi_{\varkappa_{2}}^{\prime}\left(\varkappa_{1}, \varkappa_{2}\right) \\
& +\frac{1}{2}\left\{\left(s_{1}-\varkappa_{1}\right)^{2} \varphi_{\varkappa_{1} \varkappa_{1}}^{\prime \prime}\left(\varkappa_{1}, \varkappa_{2}\right)+2\left(s_{1}-\varkappa_{1}\right)\left(s_{2}-\varkappa_{2}\right) \varphi_{\varkappa_{1} \varkappa_{2}}^{\prime \prime}\left(\varkappa_{1}, \varkappa_{2}\right)\right. \\
& \left.+\left(s_{2}-\varkappa_{2}\right)^{2} \varphi_{\varkappa_{2} \varkappa_{2}}^{\prime \prime}\left(\varkappa_{1}, \varkappa_{2}\right)\right\}+\epsilon\left(s_{1}, s_{2} ; \varkappa_{1}, \varkappa_{2}\right) \sqrt{\left(s_{1}-\varkappa_{1}\right)^{4}+\left(s_{2}-\varkappa_{2}\right)^{4}},
\end{aligned}
$$

where $\epsilon\left(s_{1}, s_{2} ; \varkappa_{1}, \varkappa_{2}\right) \in C_{\beta_{1}, \beta_{2}}\left(\mathbb{R}_{+}^{2}\right)$, for some $\beta_{1}, \beta_{2}>0$ and $\epsilon\left(s_{1}, s_{2} ; \varkappa_{1}, \varkappa_{2}\right) \rightarrow 0$, as $\left(s_{1}, s_{2}\right) \rightarrow\left(\varkappa_{1}, \varkappa_{2}\right)$. Applying the operators $\mathcal{L}_{\mathrm{m}, \mathrm{m}, \wp_{1}, \wp_{2}}\left(\cdot ; \varkappa_{1}, \varkappa_{2}, \mathrm{~b}_{1}, \mathrm{~b}_{2}\right)$ on both sides of (4.5) and using Lemma 4.1, we get

$$
\begin{aligned}
& \mathcal{L}_{\mathrm{m}, \mathrm{m}, \wp_{1}, \wp_{2}}\left(\varphi ; \varkappa_{1}, \varkappa_{2}, \mathrm{~d}_{1}, \mathrm{~d}_{2}\right)=\varphi\left(\varkappa_{1}, \varkappa_{2}\right) \mathcal{L}_{\ell, \ell, \wp_{1}, \wp_{2}}\left(e_{00} ; \varkappa_{1}, \varkappa_{2}, \mathrm{~d}_{1}, \mathrm{~d}_{2}\right) \\
& +\varphi_{\varkappa_{1}}^{\prime}\left(\varkappa_{1}, \varkappa_{2}\right) \mathcal{L}_{\ell, \ell, \wp_{1}, \wp_{2}}\left(\left(\mathrm{~s}_{1}-\varkappa_{1}\right) ; \varkappa_{1}, \varkappa_{2}, \mathrm{~d}_{1}, \mathrm{~d}_{2}\right) \\
& +\varphi_{\varkappa_{2}}^{\prime}\left(\varkappa_{1}, \varkappa_{2}\right) \mathcal{L}_{\ell, \ell, \wp_{1}, \wp_{2}}\left(\left(s_{2}-\varkappa_{2}\right) ; \varkappa_{1}, \varkappa_{2}, \mathrm{~d}_{1}, \mathrm{~d}_{2}\right) \\
& +\frac{1}{2}\left\{\varphi_{\varkappa_{1} \varkappa_{1}}^{\prime \prime}\left(\varkappa_{1}, \varkappa_{2}\right) \mathcal{L}_{\ell, \ell_{\wp_{1}, \wp_{2}}}\left(\left(\mathrm{~s}_{1}-\varkappa_{1}\right)^{2} ; \varkappa_{1}, \varkappa_{2}, \mathrm{~d}_{1}, \mathrm{~d}_{2}\right)\right. \\
& +2 \varphi_{\varkappa_{1} \varkappa_{2}}^{\prime \prime}\left(\varkappa_{1}, \varkappa_{2}\right) \mathcal{L}_{\left.\ell, \ell, \wp_{1}, \wp_{2}\right)}\left(\left(s_{1}-\varkappa_{1}\right)\left(s_{2}-\ell_{2}\right) ; \varkappa_{1}, \varkappa_{2}, \mathrm{~d}_{1}, \mathrm{~d}_{2}\right) \\
& +\varphi_{\varkappa_{2} \varkappa_{2}}^{\prime \prime}\left(\varkappa_{1}, \varkappa_{2}\right) \mathcal{L}_{\ell, \ell, \wp_{1}, \wp_{2}}\left(\left(s_{2}-\varkappa_{2}\right)^{2} ; \varkappa_{1}, \varkappa_{2}, \mathrm{~d}_{1}, \mathrm{~d}_{2}\right\} \\
& +\mathcal{L}_{\ell, \ell, \wp_{1}, \wp_{2}}\left(\epsilon\left(s_{1}, s_{2} ; \varkappa_{1}, \varkappa_{2}\right) \sqrt{\left(s_{1}-\varkappa_{1}\right)^{4}+\left(s_{2}-\varkappa_{2}\right)^{4}} ; \varkappa_{1}, \varkappa_{2}, d_{1}, d_{2}\right) .
\end{aligned}
$$

Now, using the Hölder's inequality, we obtain

$$
\begin{aligned}
\mid \mathcal{L}_{\ell, \ell, \wp_{1}, \wp_{2}}( & \left.\epsilon\left(\mathrm{s}_{1}, \mathrm{~s}_{2} ; \varkappa_{1}, \varkappa_{2}\right) \sqrt{\left(\mathrm{s}_{1}-\varkappa_{1}\right)^{4}+\left(\mathrm{s}_{2}-\varkappa_{2}\right)^{4}} ; \varkappa_{1}, \varkappa_{2}, \mathrm{~d}_{1}, \mathrm{~d}_{2}\right) \mid \\
\leqslant & \left(\mathcal{L}_{\ell, \ell, \wp_{1}, \wp_{2}}\left(\epsilon^{2}\left(\mathrm{~s}_{1}, \mathrm{~s}_{2} ; \varkappa_{1}, \varkappa_{2}\right) ; \varkappa_{1}, \varkappa_{2}, \mathrm{~d}_{1}, \mathrm{~d}_{2}\right)\right)^{\frac{1}{2}}\left(\mathcal{L}_{\ell, \ell, \wp_{1}, \wp_{2}}\left(\left(\mathrm{~s}_{1}-\varkappa_{1}\right)^{4}+\left(\mathrm{s}_{2}-\varkappa_{2}\right)^{4} ; \varkappa_{1}, \varkappa_{2}, \mathrm{~d}_{1}, \mathrm{~d}_{2}\right)\right)^{\frac{1}{2}} \\
\leqslant & \left(\mathcal{L}_{\ell, \ell, \wp_{1}, \wp_{2}}\left(\epsilon^{2}\left(\mathrm{~s}_{1}, \mathrm{~s}_{2} ; \varkappa_{1}, \varkappa_{2}\right) ; \varkappa_{1}, \varkappa_{2}, \mathrm{~d}_{1}, \mathrm{~d}_{2}\right)\right)^{\frac{1}{2}} \\
& \times\left(\mathcal{L}_{\ell, \ell, \wp_{1}, \wp_{2}}\left(\left(\mathrm{~s}_{1}-\varkappa_{1}\right)^{4} ; \varkappa_{1}, \varkappa_{2}, \mathrm{~d}_{1}, \mathrm{~d}_{2}\right)+\mathcal{L}_{\ell, \ell, \wp_{1}, \wp_{2}}\left(\left(\mathrm{~s}_{2}-\varkappa_{2}\right)^{4} ; \varkappa_{1}, \varkappa_{2}, \mathrm{~d}_{1}, \mathrm{~d}_{2}\right)\right)^{\frac{1}{2}} .
\end{aligned}
$$


From Theorem $4.4, \mathcal{L}_{\ell, \ell, \wp_{1}, \wp_{2}}\left(\epsilon^{2}\left(\mathrm{~s}_{1}, \mathrm{~s}_{2} ; \varkappa_{1}, \varkappa_{2}\right) ; \varkappa_{1}, \varkappa_{2}, \mathrm{~d}_{1}, \mathrm{~d}_{2}\right) \rightarrow 0$ as $\ell \rightarrow \infty$, uniformly on each compact subset of $\mathbb{R}_{+}^{2}$, we have

$$
\mathcal{L}_{\ell, \ell, \wp_{1}, \wp_{2}}\left(\left(s_{1}-\varkappa_{1}\right)^{4} ; \varkappa_{1}, \varkappa_{2}, \mathrm{~d}_{1}, \mathrm{~d}_{2}\right)=\mathrm{O}\left(\frac{1}{\ell^{2}}\right), \quad \mathcal{L}_{\ell, \ell, \wp_{1}, \wp_{2}}\left(\left(\mathrm{~s}_{2}-\varkappa_{2}\right)^{4} ; \varkappa_{1}, \varkappa_{2}, \mathrm{~d}_{1}, \mathrm{~d}_{2}\right)=\mathrm{O}\left(\frac{1}{\ell^{2}}\right),
$$

uniformly on each compact subset of $\mathbb{R}_{+}^{2}$, hence

$$
\lim _{\ell \rightarrow \infty} \ell \mathcal{L}_{\ell, \ell, \wp_{1}, \wp_{2}}\left(\epsilon\left(s_{1}, s_{2} ; \varkappa_{1}, \varkappa_{2}\right) \sqrt{\left(s_{1}-\varkappa_{1}\right)^{4}+\left(s_{2}-\varkappa_{2}\right)^{4}} ; \varkappa_{1}, \varkappa_{2}, \mathrm{~d}_{1}, \mathrm{~d}_{2}\right)=0,
$$

uniformly on each compact subset of $\mathbb{R}_{+}^{2}$. The required result, now follows from (4.6) on applying Remark 4.3. This completes the proof of theorem.

\section{Numerical results and discussions}

We present numerical results to demonstrate and compare the rate of convergence of the generalized bivariate Kantorovich-Szász-Charlier type operator $\mathcal{L}_{\ell_{1}, \ell_{2}, \wp_{1}, \wp_{2}}\left(\varphi ; \varkappa_{1}, \varkappa_{2}, d_{1}, d_{2}\right)$ to the function $\phi\left(\varkappa_{1}, \varkappa_{2}\right)$ with different values of parameters.

Example 5.1. Let us consider $\varphi: \mathbb{R}^{2} \rightarrow \mathbb{R}, \varphi\left(\varkappa_{1}, \varkappa_{2}\right)=\varkappa_{1}^{2} \varkappa_{2}-\varkappa_{1} \varkappa_{2}^{3}$. Let $\ell_{1}=\ell_{2}=30, \mathrm{~d}_{1}=\mathrm{d}_{2}=2$, and $\wp_{1}=\wp_{2}=1,2$, and 3 . The convergence of the generalized bivariate Kantorovich-Szász-Charlier type operator $\mathcal{L}_{\ell_{1}, \ell_{2}, \wp_{1}, \wp_{2}}\left(\varphi ; \varkappa_{1}, \varkappa_{2}, \mathrm{~d}_{1}, \mathrm{~d}_{2}\right)$ towards the function $\varphi\left(\varkappa_{1}, \varkappa_{2}\right)$, is illustrated in Figure 2 . Also, the absolute error in the approximation at certain points in the interval $\left(\varkappa_{1}, \varkappa_{2}\right) \in \mathbb{R}^{2}$ is computed in Table 2. Denote $\mathcal{E}_{\ell_{1}, \ell_{2}, \wp_{1}}^{\mathrm{d}_{1}, \mathrm{~d}_{2}, \vartheta_{2}}\left(\varphi ; \varkappa_{1}, \varkappa_{2}\right)=\left|\mathcal{L}_{\ell_{1}, \ell_{2}, \wp_{1}, \wp_{2}}\left(\varphi ; \varkappa_{1}, \varkappa_{2}, \mathrm{~d}_{1}, \mathrm{~d}_{2}\right)-\varphi\left(\varkappa_{1}, \varkappa_{2}\right)\right|$, the error function of approximation by $\mathcal{L}_{\ell_{1}, \ell_{2}, \vartheta_{1}, \vartheta_{2}}\left(\varphi ; \varkappa_{1}, \varkappa_{2}, d_{1}, d_{2}\right)$ operators. The error of approximation is illustrated in Figure 3 . We notice that the error in the approximation of the function by the operators becomes smaller as $\wp_{1}$ and $\wp_{2}$ increase. $[\mathrm{h} !]$

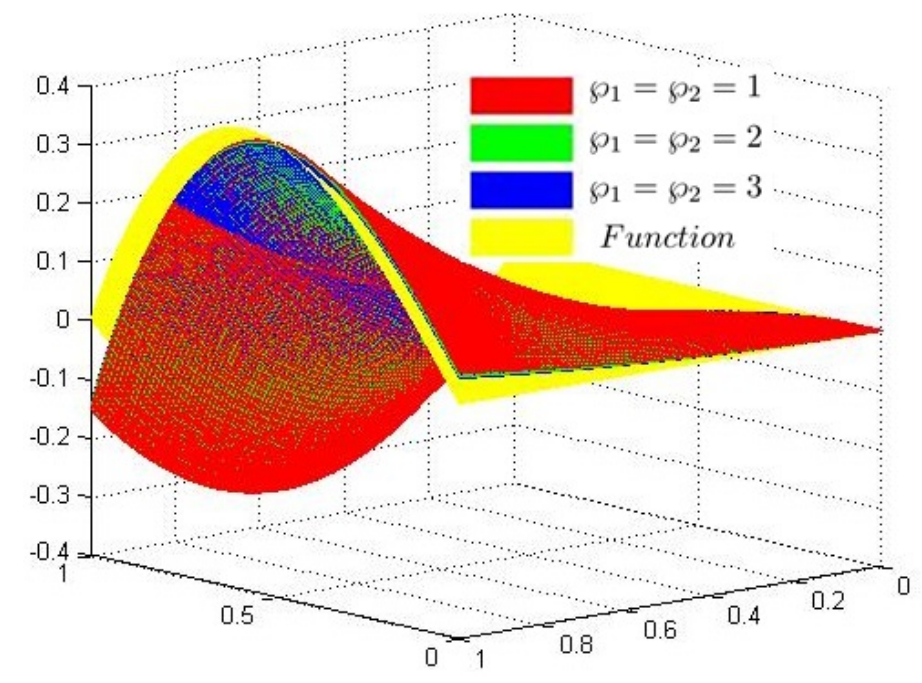

Figure 2: The convergence of operators $\mathcal{L}_{\ell_{1}, \ell_{2}, \wp_{1}, \wp_{2}}\left(\varphi ; \varkappa_{1}, \varkappa_{2}, d_{1}, d_{2}\right)$ to the function $\varphi\left(\varkappa_{1}, \varkappa_{2}\right)$.

\section{GBS operator associated to $\mathcal{L}_{\ell_{1}, \ell_{2}, \wp_{1}, \wp_{2}}\left(\cdot ; \varkappa_{1}, \varkappa_{2}, d_{1}, d_{2}\right)$}

In [6, 7], Bögel proposed the concepts of B-continuous and B-differentiable functions. Later, Dobrescu and Matei [12] discussed the approximation of B-continuous functions on a bounded interval by the GBS of bivariate generalization of Bernstein polynomials. Subsequently, Badea and Cottin [2] 


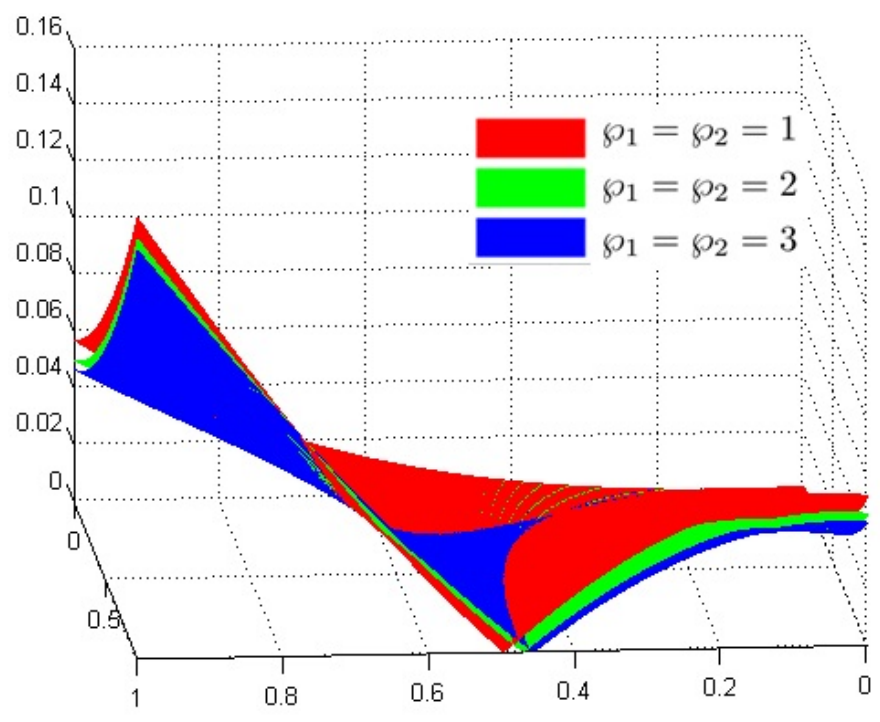

Figure 3: Error of approximation.

Table 2: Error of approximation for operators $\mathcal{L}_{\ell_{1}, \ell_{2}, \wp_{1}, \wp_{2}}\left(\varphi ; \varkappa_{1}, \varkappa_{2}, \mathrm{~d}_{1}, \mathrm{~d}_{2}\right)$ to the function $\varphi\left(\varkappa_{1}, \varkappa_{2}\right)$.

\begin{tabular}{|c|c|c|c|}
\hline$\left(\varkappa_{1}, \varkappa_{2}\right)$ & $\mathcal{E}_{30,30,1}^{2,2,1}\left(\varphi ; \varkappa_{1}, \varkappa_{2}\right)$ & $\mathcal{E}_{30,30,2}^{2,2,2}\left(\varphi ; \varkappa_{1}, \varkappa_{2}\right)$ & $\mathcal{E}_{30,30,3}^{2,2,3}\left(\varphi ; \varkappa_{1}, \varkappa_{2}\right)$ \\
\hline$(0.1,0.1)$ & 0.0020 & 0.0017 & 0.0016 \\
\hline$(0.15,0.25)$ & 0.0027 & 0.0024 & 0.0022 \\
\hline$(0.20,0.35)$ & 0.0016 & 0.0014 & 0.0012 \\
\hline$(0.4,0.4)$ & 0.0040 & 0.0031 & 0.0026 \\
\hline$(0.5,0.55)$ & 0.0086 & 0.0081 & 0.0074 \\
\hline$(0.6,0.6)$ & 0.0162 & 0.0157 & 0.0150 \\
\hline$(0.85,0.9)$ & 0.0985 & 0.0941 & 0.0919 \\
\hline$(0.95,0.95)$ & 0.1258 & 0.1203 & 0.1175 \\
\hline
\end{tabular}

established Korovkin type theorems for GBS operators. We introduce the GBS case of the operators $\mathcal{L}_{\ell_{1}, \ell_{2}, \wp_{1}, \wp_{2}}\left(\cdot ; \varkappa_{1}, \varkappa_{2}, \mathrm{~d}_{1}, \mathrm{~d}_{2}\right)$. We begin with some basic definitions and notations. For further details, we refer to the book [14].

Let $X_{1}$ and $X_{2}$ be any subsets of $\mathbb{R}$. A function $\varphi: X_{1} \times X_{2} \rightarrow \mathbb{R}$ is called B-continuous (Bögel continuous) at a point $\left(\varkappa_{1}, \varkappa_{2}\right) \in X_{1} \times X_{2}$ if

$$
\lim _{\left(s_{1}, s_{2}\right) \rightarrow\left(\varkappa_{1}, \varkappa_{2}\right)} \Delta \varphi\left[\left(s_{1}, s_{2}\right) ;\left(\varkappa_{1}, \varkappa_{2}\right)\right]=0,
$$

where $\Delta \varphi\left[\left(s_{1}, s_{2}\right) ;\left(\varkappa_{1}, \varkappa_{2}\right)\right]=\varphi\left(\varkappa_{1}, \varkappa_{2}\right)-\varphi\left(\varkappa_{1}, s_{2}\right)-\varphi\left(s_{1}, \varkappa_{2}\right)+\varphi\left(s_{1}, s_{2}\right)$ denotes the mixed difference.

A function $\varphi: X_{1} \times X_{2} \rightarrow \mathbb{R}$ is called B-continuous in $X_{1} \times X_{2}$ if it is B-continuous at each point of $X_{1} \times X_{2}$. Let $C_{b}\left(X_{1} \times X_{2}\right)$ denotes the space of all B-continuous functions in $X_{1} \times X_{2}$.

A function $\varphi: X_{1} \times X_{2} \rightarrow \mathbb{R}$ is called uniformly B-continuous in $X_{1} \times X_{2}$ if for a given $\varepsilon>0 \exists \delta>0$ such that $\left|\Delta \varphi\left[\left(\mathrm{s}_{1}, \mathrm{~s}_{2}\right):\left(\varkappa_{1}, \varkappa_{2}\right)\right]\right|<\varepsilon$ whenever $\sqrt{\left(\mathrm{s}_{1}-\varkappa_{1}\right)^{2}+\left(\mathrm{s}_{2}-\varkappa_{2}\right)^{2}}<\delta$. Let $\overline{\mathrm{C}}_{\mathrm{b}}\left(\mathrm{X}_{1} \times \mathrm{X}_{2}\right)$ denote the space of uniformly B-continuous functions on $\mathbb{R}_{+}^{2}$. A function $\varphi: X_{1} \times X_{2} \rightarrow \mathbb{R}$ is called B-bounded on $X_{1} \times X_{2}$ if there exists $M>0$ such that

$$
\left|\Delta \varphi\left[\left(s_{1}, s_{2}\right) ;\left(\varkappa_{1}, \varkappa_{2}\right)\right]\right| \leqslant M,
$$

for every $\left(\varkappa_{1}, \varkappa_{2}\right),\left(s_{1}, s_{2}\right) \in X_{1} \times X_{2}$.

Let $\mathrm{B}_{\mathrm{b}}\left(\mathrm{X}_{1} \times \mathrm{X}_{2}\right)$ denote all B-bounded functions on $\mathrm{X}_{1} \times \mathrm{X}_{2} \rightarrow \mathbb{R}$, equipped with the norm

$$
\|\varphi\|_{\mathrm{B}}=\sup _{\left(\varkappa_{1}, \varkappa_{2}\right),\left(\mathrm{s}_{1}, \mathrm{~s}_{2}\right) \in \mathrm{X}_{1} \times \mathrm{X}_{2}}\left|\Delta \varphi\left[\left(\mathrm{s}_{1}, \mathrm{~s}_{2}\right) ;\left(\varkappa_{1}, \varkappa_{2}\right)\right]\right| .
$$


Let $B\left(X_{1} \times X_{2}\right)$ and $C\left(X_{1} \times X_{2}\right)$ denote the space of all bounded functions and the space of all continuous functions on $X_{1} \times X_{2}$ respectively endowed with the sup-norm $\|\cdot\|_{\infty}$. It is known that $C\left(X_{1} \times X_{2}\right) \subset C_{b}\left(X_{1} \times\right.$ $\left.\mathrm{X}_{2}\right)[8]$.

A function $\varphi: X_{1} \times X_{2} \rightarrow \mathbb{R}$ is called a B-differentiable (Bögel differentiable) function at $\left(\varkappa_{1}, \varkappa_{2}\right) \in$ $X_{1} \times X_{2}$ if the limit

$$
\lim _{\left(s_{1}, s_{2}\right) \rightarrow\left(\varkappa_{1}, \varkappa_{2}\right)} \frac{\Delta \varphi\left[\left(s_{1}, s_{2}\right) ;\left(\varkappa_{1}, \varkappa_{2}\right)\right]}{\left(s_{1}-\varkappa_{1}\right)\left(s_{2}-\varkappa_{2}\right)}
$$

exists and finite.

The limit is said to be the B-differential of $\varphi$ at the point $\left(\varkappa_{1}, \varkappa_{2}\right)$ and is denoted by $\mathrm{D}_{\mathrm{B}}\left(\varphi ; \varkappa_{1}, \varkappa_{2}\right)$ and the space of all B-differentiable functions is denoted by $D_{b}\left(X_{1} \times X_{2}\right)$.

The mixed modulus of smoothness of $\varphi \in C_{b}\left(X_{1} \times X_{2}\right)$ is defined as

$$
\omega_{\mathrm{B}}\left(\varphi ; \delta_{1}, \delta_{2}\right)=\sup \left\{\left|\Delta \varphi\left[\left(s_{1}, s_{2}\right) ;\left(\varkappa_{1}, \varkappa_{2}\right)\right]\right|:\left|\varkappa_{1}-s_{1}\right|<\delta_{1},\left|\varkappa_{2}-s_{2}\right|<\delta_{2}\right\},
$$

for all $\left(\varkappa_{1}, \varkappa_{2}\right),\left(s_{1}, s_{2}\right) \in X_{1} \times X_{2}$ and for any $\left(\delta_{1}, \delta_{2}\right) \in(0, \infty) \times(0, \infty)$ with $\omega_{B}:(0, \infty) \times(0, \infty) \rightarrow \mathbb{R}$. The basic properties of $\omega_{B}$ were obtained by Badea et al. in $[3,4]$ which are similar to the properties of the usual modulus of continuity.

For every $\varphi \in C_{b}\left(\mathbb{R}_{+}^{2}\right)$, the GBS operators associated with the operators $\mathcal{L}_{\ell_{1}, \ell_{2}, \wp_{1}, \wp_{2}}\left(\varphi ; \varkappa_{1}, \varkappa_{2}, d_{1}, d_{2}\right)$ is defined by:

$$
\begin{aligned}
\mathcal{G}_{\ell_{1}, \ell_{2}, \wp_{1}, \wp_{2}}\left(\varphi ; \varkappa_{1}, \varkappa_{2}, \mathrm{~d}_{1}, \mathrm{~d}_{2}\right)= & \mathcal{L}_{\ell_{1}, \ell_{2}, \wp_{1}, \wp_{2}}\left(\varphi\left(s_{1}, \varkappa_{2}\right)+\varphi\left(\varkappa_{1}, s_{2}\right)-\varphi\left(s_{1}, s_{2}\right) ; \varkappa_{1}, \varkappa_{2}, \mathrm{~d}_{1}, \mathrm{~d}_{2}\right) \\
= & e^{-1}\left(1-\frac{1}{\mathrm{~d}_{1}}\right)^{\left(\mathrm{d}_{1}-1\right) \ell_{1} \varkappa_{1}} \mathrm{e}^{-1}\left(1-\frac{1}{\mathrm{~d}_{2}}\right)^{\left(\mathrm{d}_{2}-1\right) \ell_{2} \varkappa_{2}} \\
& \times \sum_{\mathrm{j}_{1}=0}^{\infty} \sum_{\mathrm{j}_{2}=0}^{\infty} \frac{\mathrm{C}_{\mathrm{j}_{1}}^{\left(\mathrm{d}_{1}\right)}\left(-\left(\mathrm{d}_{1}-1\right) \ell_{1} \varkappa_{1}\right)}{\mathrm{j}_{1} !} \frac{\mathrm{C}_{\mathrm{j}_{2}}^{\left(\mathrm{d}_{2}\right)}\left(-\left(\mathrm{d}_{2}-1\right) \ell_{2} \varkappa_{2}\right)}{j_{2} !} \\
& \times \int_{0}^{1} \int_{0}^{1}\left[\varphi\left(\frac{s_{1}^{\wp_{1}}+j_{1}}{\ell_{1}+1}, \varkappa_{2}\right)+\varphi\left(\varkappa_{1} \frac{s_{2}^{\wp_{2}}+j_{2}}{\ell_{2}+1}\right)-\varphi\left(\frac{s_{1}^{\wp_{1}}+j_{1}}{\ell_{1}+1}, \frac{s_{2}^{\wp_{2}}+j_{2}}{\ell_{2}+1}\right)\right] \mathrm{d} s_{1} d s_{2} .
\end{aligned}
$$

From the above definition, it is clear that $\mathcal{G}_{\ell_{1}, \ell_{2}, \wp_{1}, \wp_{2}}$ is a linear operator.

Theorem 6.1. For every $\varphi \in \overline{\mathrm{C}}_{\mathrm{b}}\left(\mathbb{R}_{+}^{2}\right)$, at each point $\left(\varkappa_{1}, \varkappa_{2}\right) \in \mathbb{R}_{+}^{2}$, the operator $\mathcal{G}_{\ell_{1}, \ell_{2}, \wp_{1}, \wp_{2}}\left(\varphi ; \varkappa_{1}, \varkappa_{2}\right)$ verifies the following inequality

$$
\left|\mathcal{G}_{\ell_{1}, \ell_{2}, \wp_{1}, \wp_{2}}\left(\varphi ; \varkappa_{1}, \varkappa_{2}, \mathrm{~d}_{1}, \mathrm{~d}_{2}\right)-\varphi\left(\varkappa_{1}, \varkappa_{2}\right)\right| \leqslant 4 \omega_{\mathrm{B}}\left(\varphi ; \sqrt{\mu_{\ell_{1}, \wp_{1}, \mathrm{~d}_{1}, 2}\left(\varkappa_{1}\right)}, \sqrt{\mu_{\ell_{2}, \wp_{2}, \mathrm{~d}_{2}, 2}\left(\varkappa_{2}\right)}\right) .
$$

Proof. Using the definition of $\omega_{\mathrm{B}}\left(\varphi ; \delta_{1}, \delta_{2}\right)$ and the elementary inequality, we have

$$
\begin{aligned}
\left|\Delta \varphi\left[\left(s_{1}, s_{2}\right) ;\left(\varkappa_{1}, \varkappa_{2}\right)\right]\right| & \leqslant \omega_{\mathrm{B}}\left(\varphi ;\left|s_{1}-\varkappa_{1}\right|,\left|s_{2}-\varkappa_{2}\right|\right) \leqslant\left(1+\frac{\left|s_{1}-\varkappa_{1}\right|}{\delta_{1}}\right)\left(1+\frac{\left|s_{2}-\varkappa_{2}\right|}{\delta_{2}}\right) \omega_{\mathrm{B}}\left(\varphi ; \delta_{1}, \delta_{2}\right) \\
& \leqslant\left(1+\frac{\left|s_{1}-\varkappa_{1}\right|}{\delta_{1}}+\frac{\left|s_{2}-\varkappa_{2}\right|}{\delta_{2}}+\frac{1}{\delta_{1} \delta_{2}}\left(\left|s_{1}-\varkappa_{1}\right|\left|s_{2}-\varkappa_{2}\right|\right)\right) \omega_{\mathrm{B}}\left(\varphi ; \delta_{1}, \delta_{2}\right),
\end{aligned}
$$

for every $\left(s_{1}, s_{2}\right) \in \mathbb{R}_{+}^{2}$ and any $\delta_{1}, \delta_{2}>0$. From (6.1), by using the definition of the mixed difference $\Delta \varphi\left[\left(s_{1}, s_{2}\right) ;\left(\varkappa_{1}, \varkappa_{2}\right)\right]$ and the inequality (6.2), we get

$$
\begin{aligned}
\mid \mathcal{G}_{\varkappa_{1}, \varkappa_{2}, \wp_{1}, \wp_{2}} & \left(\varphi ; \varkappa_{1}, \varkappa_{2}, \mathrm{~d}_{1}, \mathrm{~d}_{2}\right)-\varphi\left(\varkappa_{1}, \varkappa_{2}\right) \mid \\
& \leqslant \mathcal{L}_{\ell_{1}, \ell_{2}, \wp_{1}, \wp_{2}}\left(\left|\Delta \varphi\left[\left(\mathrm{s}_{1}, \mathrm{~s}_{2}\right) ;\left(\varkappa_{1}, \varkappa_{2}\right)\right]\right| ; \varkappa_{1}, \varkappa_{2}, \mathrm{~d}_{1}, \mathrm{~d}_{2}\right) \\
& \leqslant \omega_{\mathrm{B}}\left(\varphi ; \delta_{1}, \delta_{2}\right)\left[\mathcal{L}_{\ell_{1}, \ell_{2}, \wp_{1}, \wp_{2}}\left(e_{00} ; \varkappa_{1}, \varkappa_{2}, \mathrm{~d}_{1}, \mathrm{~d}_{2}\right)+\frac{1}{\delta_{1}} \mathcal{L}_{\ell_{1}, \ell_{2}, \wp_{1}, \wp_{2}}\left(\left|\mathrm{~s}_{1}-\varkappa_{1}\right| ; \varkappa_{1}, \varkappa_{2}, \mathrm{~d}_{1}, \mathrm{~d}_{2}\right)\right.
\end{aligned}
$$




$$
\begin{aligned}
& +\frac{1}{\delta_{2}} \mathcal{L}_{\ell_{1}, \ell_{2}, \wp_{1}, \wp_{2}}\left(\left|\mathrm{~s}_{2}-\varkappa_{2}\right| ; \varkappa_{1}, \varkappa_{2}, \mathrm{~d}_{1}, \mathrm{~d}_{2}\right) \\
& \left.+\frac{1}{\delta_{1} \delta_{2}} \mathcal{L}_{\ell_{1}, \ell_{2}, \wp_{1}, \wp_{2}}\left(\left|\mathrm{~s}_{1}-\varkappa_{1}\right| ; \varkappa_{1}, \varkappa_{2}, \mathrm{~d}_{1}, \mathrm{~d}_{2}\right) \mathcal{L}_{\ell_{1}, \ell_{2}, \wp_{1}, \wp_{2}}\left(\left|\mathrm{~s}_{2}-\varkappa_{2}\right| ; \varkappa_{1}, \varkappa_{2}, \mathrm{~d}_{1}, \mathrm{~d}_{2}\right)\right] .
\end{aligned}
$$

Now, using Lemma 4.1 and applying the Cauchy-Schwarz inequality, we get

$$
\begin{aligned}
&\left|\mathcal{G}_{\ell_{1}, \ell_{2}, \wp_{1}, \wp_{2}}\left(\varphi ; \varkappa_{1}, \varkappa_{2}, \mathrm{~d}_{1}, \mathrm{~d}_{2}\right)-\varphi\left(\varkappa_{1}, \varkappa_{2}\right)\right| \\
& \leqslant \omega_{\mathrm{B}}\left(\varphi ; \delta_{1}, \delta_{2}\right)\left[1+\frac{1}{\delta_{1}} \sqrt{\mathcal{L}_{\ell_{1}, \ell_{2}, \wp_{1}, \wp_{2}}\left(\left(\mathrm{~s}_{1}-\varkappa_{1}\right)^{2} ; \varkappa_{1}, \varkappa_{2}, \mathrm{~d}_{1}, \mathrm{~d}_{2}\right)}\right. \\
&+\frac{1}{\delta_{2}} \sqrt{\mathcal{L}_{\ell_{1}, \ell_{2}, \wp_{1}, \wp_{2}}\left(\left(s_{2}-\varkappa_{2}\right)^{2} ; \varkappa_{1}, \varkappa_{2}, \mathrm{~d}_{1}, \mathrm{~d}_{2}\right)} \\
&\left.+\frac{1}{\delta_{1} \delta_{2}} \sqrt{\mathcal{L}_{\ell_{1}, \ell_{2}, \wp_{1}, \wp_{2}}\left(\left(\mathrm{~s}_{1}-\varkappa_{1}\right)^{2} ; \varkappa_{1}, \varkappa_{2}, \mathrm{~d}_{1}, \mathrm{~d}_{2}\right) \mathcal{L}_{\ell_{1}, \ell_{2}, \wp_{1}, \wp_{2}}\left(\left(s_{2}-\varkappa_{2}\right)^{2} ; \varkappa_{1}, \varkappa_{2}, \mathrm{~b}_{1}, \mathrm{~b}_{2}\right)}\right],
\end{aligned}
$$

which leads us to the required result on choosing $\delta_{1}=\sqrt{\mu_{\ell_{1}, \wp_{1}, \mathrm{~d}_{1}, 2}\left(\varkappa_{1}\right)}$ and $\delta_{2}=\sqrt{\mu_{\ell_{2}, \wp_{2}, \mathrm{~d}_{2}, 2}\left(\varkappa_{2}\right)}$.

\section{Lipschitz class of B-continuous functions}

For $0<\alpha_{1}, \alpha_{2} \leqslant 1$ and $\varphi \in B_{\mathfrak{b}}\left(\mathbb{R}_{+}^{2}\right)$, the Lipschitz class $\operatorname{Lip}_{M}^{*}\left(\alpha_{1}, \alpha_{2}\right)$ of $\varphi$ is defined as follows:

$$
\operatorname{Lip}_{M}^{*}\left(\alpha_{1}, \alpha_{2}\right)=\left\{\varphi \in B_{b}\left(\mathbb{R}_{+}^{2}\right):\left|\Delta \varphi\left[\left(s_{1}, s_{2}\right) ;\left(\varkappa_{1}, \varkappa_{2}\right)\right]\right| \leqslant M\left|s_{1}-\varkappa_{1}\right|^{\alpha_{1}}\left|s_{2}-\varkappa_{2}\right|^{\alpha_{2}}\right\},
$$

for all $\left(s_{1}, s_{2}\right),\left(\varkappa_{1}, \varkappa_{2}\right) \in \mathbb{R}_{+}^{2}$ and $\mathrm{M}$ is a positive constant.

In our next result, we determine the degree of approximation for the operators $\mathcal{G}_{\ell_{1}, \ell_{2}, \wp_{1}, \wp_{2}}\left(\varphi ; \varkappa_{1}, \varkappa_{2}, d_{1}, d_{2}\right)$ by means of the class $\operatorname{Lip}_{M}^{*}\left(\alpha_{1}, \alpha_{2}\right)$ class of Bögel continuous functions.

Theorem 7.1. Let $\varphi \in \operatorname{Lip}_{M}^{*}\left(\alpha_{1}, \alpha_{2}\right)$ and $\alpha_{1}, \alpha_{2} \in(0,1]$, then we have

$$
\left|\mathcal{G}_{\ell_{1}, \ell_{2}, \wp_{1}, \wp_{2}}\left(\varphi ; \varkappa_{1}, \varkappa_{2}, \mathrm{~d}_{1}, \mathrm{~d}_{2}\right)-\varphi\left(\varkappa_{1}, \varkappa_{2}\right)\right| \leqslant M\left(\mu_{\ell_{1}, \wp_{1}, \mathrm{~d}_{1}, 2}\left(\varkappa_{1}\right)\right)^{\frac{\alpha_{1}}{2}}\left(\mu_{\ell_{2}, \wp_{2}, \mathrm{~d}_{2}, 2}\left(\varkappa_{2}\right)\right)^{\frac{\alpha_{2}}{2}} .
$$

Proof. From the definition of the mixed difference $\Delta \varphi\left[\left(s_{1}, s_{2}\right) ;\left(\varkappa_{1}, \varkappa_{2}\right)\right],(6.1)$ and by our hypothesis, we may write

$$
\begin{aligned}
\left|\mathcal{G}_{\ell_{1}, \ell_{2}, \wp_{1}, \wp_{2}}\left(\varphi ; \varkappa_{1}, \varkappa_{2}, \mathrm{~d}_{1}, \mathrm{~d}_{2}\right)-\varphi\left(\varkappa_{1}, \varkappa_{2}\right)\right| \leqslant & \mathcal{L}_{\ell_{1}, \ell_{2}, \wp_{1}, \wp_{2}}\left(\left|\Delta \varphi\left[\left(\mathrm{s}_{1}, \mathrm{~s}_{2}\right) ;\left(\varkappa_{1}, \varkappa_{2}\right)\right]\right| ; \varkappa_{1}, \varkappa_{2}, \mathrm{~d}_{1}, \mathrm{~d}_{2}\right) \\
\leqslant & M \mathcal{L}_{\ell_{1}, \ell_{2}, \wp_{1}, \wp_{2},}\left(\left|\mathrm{~s}_{1}-\varkappa_{1}\right|^{\alpha_{1}}\left|\mathrm{~s}_{2}-\varkappa_{2}\right|^{\alpha_{2}} ; \varkappa_{1}, \varkappa_{2}, \mathrm{~d}_{1}, \mathrm{~d}_{2}\right) \\
= & M \mathcal{L}_{\ell_{1}, \ell_{2}, \wp_{1}, \wp_{2}}\left(\left|\mathrm{~s}_{1}-\varkappa_{1}\right|^{\alpha_{1}} ; \varkappa_{1}, \varkappa_{2}, \mathrm{~d}_{1}, \mathrm{~d}_{2}\right) \\
& \times \mathcal{L}_{\ell_{1}, \ell_{2}, \wp_{1}, \wp_{2}}\left(\left|\mathrm{~s}_{2}-\varkappa_{2}\right|^{\alpha_{2}} ; \varkappa_{1}, \varkappa_{2}, \mathrm{~d}_{1}, \mathrm{~d}_{2}\right) .
\end{aligned}
$$

Now, using the Hölder's inequality with $p_{1}=\frac{2}{\alpha_{1}}, q_{1}=\frac{2}{2-\alpha_{1}}$, and $p_{2}=\frac{2}{\alpha_{2}}, q_{2}=\frac{2}{2-\alpha_{2}}$, we are led to

$$
\begin{aligned}
&\left|\mathcal{G}_{\ell_{1}, \ell_{2}, \wp_{1}, \wp_{2}}\left(\varphi ; \varkappa_{1}, \varkappa_{2}, \mathrm{~d}_{1}, \mathrm{~d}_{2}\right)-\varphi\left(\varkappa_{1}, \varkappa_{2}\right)\right| \\
& \leqslant M\left(\mathcal{L}_{\ell_{1}, \ell_{2}, \wp_{1}, \wp_{2}}\left(\left(\mathrm{~s}_{1}-\varkappa_{1}\right)^{2} ; \varkappa_{1}, \varkappa_{2}, \mathrm{~d}_{1}, \mathrm{~d}_{2}\right)\right)^{\frac{\alpha_{1}}{2}}\left(\mathcal{L}_{\ell_{1}, \ell_{2}, \wp_{1}, \wp_{2}}\left(e_{00} ; \varkappa_{1}, \varkappa_{2}, \mathrm{~d}_{1}, \mathrm{~d}_{2}\right)\right)^{\frac{2-\alpha_{1}}{2}} \\
& \times\left(\mathcal{L}_{\ell_{1}, \ell_{2}, \wp_{1}, \wp_{2}}\left(\left(\mathrm{~s}_{2}-\varkappa_{2}\right)^{2} ; \varkappa_{1}, \varkappa_{2}, \mathrm{~d}_{1}, \mathrm{~d}_{2}\right)\right)^{\frac{\alpha_{2}}{2}} \mathcal{L}_{\ell_{1}, \ell_{2}, \wp_{1}, \wp_{2}}\left(\left(e_{00} ; \varkappa_{1}, \varkappa_{2}, \mathrm{~d}_{1}, \mathrm{~d}_{2}\right)\right)^{\frac{2-\alpha_{2}}{2}} .
\end{aligned}
$$

In view of Lemma 4.1, the desired result is immediate.

Theorem 7.2. For $\varphi \in \mathrm{D}_{\mathrm{b}}\left(\mathbb{R}_{+}^{2}\right)$ with $\mathrm{D}_{\mathrm{B}} \varphi \in \overline{\mathrm{C}}_{\mathrm{b}}\left(\mathbb{R}_{+}^{2}\right) \cap \mathrm{B}\left(\mathbb{R}_{+}^{2}\right)$ and each $\left(\varkappa_{1}, \varkappa_{2}\right) \in \mathbb{R}_{+}^{2}$, we have

$$
\left|\mathcal{G}_{\ell_{1}, \ell_{2}, \wp_{1}, \wp_{2}}\left(\varphi ; \varkappa_{1}, \varkappa_{2}, \mathrm{~d}_{1}, \mathrm{~d}_{2}\right)-\varphi\left(\varkappa_{1}, \varkappa_{2}\right)\right| \leqslant \frac{\mathrm{C}}{\sqrt{\ell_{1} \ell_{2}}}\left[\left\|\mathrm{D}_{\mathrm{B}} \varphi\right\|_{\infty}+\omega\left(\mathrm{D}_{\mathrm{B}} \varphi ; \ell_{1}^{-\frac{1}{2}}, \ell_{2}^{-\frac{1}{2}}\right)\right] .
$$


Proof. Since $\varphi \in \mathrm{D}_{\mathrm{b}}\left(\mathbb{R}_{+}^{2}\right)$, we can write

$$
\begin{aligned}
\mathrm{D}_{\mathrm{B}} \varphi\left(\varkappa_{1}, \varkappa_{2}\right) & =\lim _{\left(s_{1}, s_{2}\right) \rightarrow\left(\varkappa_{1}, \varkappa_{2}\right)} \frac{\Delta \varphi\left[\left(s_{1}, s_{2}\right) ;\left(\varkappa_{1}, \varkappa_{2}\right)\right]}{\left(s_{1}-\varkappa_{1}\right)\left(s_{2}-\varkappa_{2}\right)} \\
& \Rightarrow
\end{aligned}
$$$$
\Delta \varphi\left[\left(s_{1}, s_{2}\right) ;\left(\varkappa_{1}, \varkappa_{2}\right)\right]=\left(s_{1}-\varkappa_{1}\right)\left(s_{2}-\varkappa_{2}\right) D_{\mathrm{B}} \varphi\left(\xi_{1}, \xi_{2}\right) \text {, where } \varkappa_{1}<\xi_{1}<s_{1} ; \varkappa_{2}<\xi_{2}<s_{2} .
$$

Now, using the following relation

$$
\mathrm{D}_{\mathrm{B}} \varphi\left(\xi_{1}, \xi_{2}\right)=\Delta \mathrm{D}_{\mathrm{B}} \varphi\left[\left(\xi_{1}, \xi_{2}\right) ;\left(\varkappa_{1}, \varkappa_{2}\right)\right]+\mathrm{D}_{\mathrm{B}} \varphi\left(\xi_{1}, \varkappa_{2}\right)+\mathrm{D}_{\mathrm{B}} \varphi\left(\varkappa_{1}, \xi_{2}\right)-\mathrm{D}_{\mathrm{B}} \varphi\left(\varkappa_{1}, \varkappa_{2}\right),
$$

in view of $\mathrm{D}_{\mathrm{B}} \varphi \in \overline{\mathrm{C}}_{\mathrm{b}}\left(\mathbb{R}_{+}^{2}\right) \cap \mathrm{B}\left(\mathbb{R}_{+}^{2}\right)$, we obtain

$$
\begin{aligned}
\mid \mathcal{L}_{\ell_{1}, \ell_{2}, \wp_{1}, \wp_{2}}( & \left.\Delta \varphi\left[\left(s_{1}, s_{2}\right) ;\left(\varkappa_{1}, \varkappa_{2}\right)\right] ; \varkappa_{1}, \varkappa_{2}, \mathrm{~d}_{1}, \mathrm{~d}_{2}\right) \mid \\
= & \left|\mathcal{L}_{\ell_{1}, \ell_{2}, \wp_{1}, \wp_{2}}\left(\left(\mathrm{~s}_{1}-\varkappa_{1}\right)\left(\mathrm{s}_{2}-\varkappa_{2}\right) \mathrm{D}_{\mathrm{B}} \varphi\left(\xi_{1}, \xi_{2}\right) ; \varkappa_{1}, \varkappa_{2}, \mathrm{~d}_{1}, \mathrm{~d}_{2}\right)\right| \\
\leqslant & \mathcal{L}_{\ell_{1}, \ell_{2}, \wp_{1}, \wp_{2}}\left(\left|s_{1}-\varkappa_{1}\right|\left|s_{2}-\varkappa_{2}\right|\left|\Delta \mathrm{D}_{\mathrm{B}} \varphi\left[\left(\xi_{1}, \xi_{2}\right) ;\left(\varkappa_{1}, \varkappa_{2}\right)\right]\right| ; \varkappa_{1}, \varkappa_{2}, \mathrm{~d}_{1}, \mathrm{~d}_{2}\right) \\
& +\mathcal{L}_{\ell_{1}, \ell_{2}, \wp_{1}, \wp_{2}}\left(\left|s_{1}-\varkappa_{1}\right|\left|s_{2}-\varkappa_{2}\right|\left(\left|\mathrm{D}_{\mathrm{B}} \varphi\left(\xi_{1}, \varkappa_{2}\right)\right|+\left|\mathrm{D}_{\mathrm{B}} \varphi\left(\varkappa_{1}, \xi_{2}\right)\right|+\left|\mathrm{D}_{\mathrm{B}} \varphi\left(\varkappa_{1}, \varkappa_{2}\right)\right|\right) ; \varkappa_{1}, \varkappa_{2}, \mathrm{~d}_{1}, \mathrm{~d}_{2}\right) \\
\leqslant & \mathcal{L}_{\ell_{1}, \ell_{2}, \wp_{1}, \wp_{2}}\left(\left|s_{1}-\varkappa_{1}\right|\left|s_{2}-\varkappa_{2}\right| \omega_{\mathrm{B}}\left(\mathrm{D}_{\mathrm{B}} \varphi ;\left|\xi_{1}-\varkappa_{1}\right|,\left|\xi_{2}-\varkappa_{2}\right|\right) ; \varkappa_{1}, \varkappa_{2}, \mathrm{~d}_{1}, \mathrm{~d}_{2}\right) \\
& +3\left\|\mathrm{D}_{\mathrm{B}} \varphi\right\|_{\infty} \mathcal{L}_{\ell_{1}, \ell_{2}, \wp_{1}, \wp_{2}}\left(\left|s_{1}-\varkappa_{1} \| s_{2}-\varkappa_{2}\right| ; \varkappa_{1}, \varkappa_{2}, \mathrm{~d}_{1}, \mathrm{~d}_{2}\right) .
\end{aligned}
$$

Hence taking into account

$$
\begin{aligned}
\omega_{\mathrm{B}}\left(\mathrm{D}_{\mathrm{B}} \varphi ;\left|\xi_{1}-\varkappa_{1}\right|,\left|\xi_{2}-\varkappa_{2}\right|\right) & \leqslant \omega_{\mathrm{B}}\left(\mathrm{D}_{\mathrm{B}} \varphi ;\left|s_{1}-\varkappa_{1}\right|,\left|s_{2}-\varkappa_{2}\right|\right) \\
& \leqslant\left(1+\frac{\left|s_{1}-\varkappa_{1}\right|}{\delta_{1}}\right)\left(1+\frac{\left|s_{2}-\varkappa_{2}\right|}{\delta_{2}}\right) \omega_{\mathrm{B}}\left(D_{\mathrm{B}} \varphi ; \delta_{1}, \delta_{2}\right),
\end{aligned}
$$

for any $\delta_{1}, \delta_{2}>0$ and applying the Cauchy-Schwarz inequality, we obtain

$$
\begin{aligned}
\mathcal{G}_{\ell_{1}, \ell_{2}, \wp_{1}, \wp_{2}}( & \left.\varphi ; \varkappa_{1}, \varkappa_{2}, d_{1}, d_{2}\right)-\varphi\left(\varkappa_{1}, \varkappa_{2}\right) \mid \\
\leqslant & 3\left\|D_{B} \varphi\right\|_{\infty} \sqrt{\mathcal{L}_{\ell_{1}, \ell_{2}, \wp_{1}, \wp_{2}}\left(\left(s_{1}-\varkappa_{1}\right)^{2}\left(s_{2}-\varkappa_{2}\right)^{2} ; \varkappa_{1}, \varkappa_{2}, d_{1}, d_{2}\right)} \\
& +\omega_{B}\left(D_{B} \varphi ; \delta_{1}, \delta_{2}\right)\left[\sqrt{\mathcal{L}_{\ell_{1}, \ell_{2}, \wp_{1}, \wp_{2}}\left(\left(s_{1}-\varkappa_{1}\right)^{2}\left(s_{2}-\varkappa_{2}\right)^{2} ; \varkappa_{1}, \varkappa_{2}, d_{1}, d_{2}\right)}\right. \\
& +\frac{1}{\delta_{1}} \sqrt{\mathcal{L}_{\ell_{1}, \ell_{2}, \wp_{1}, \wp_{2}}\left(\left(s_{1}-\varkappa_{1}\right)^{4}\left(s_{2}-\varkappa_{2}\right)^{2} ; \varkappa_{1}, \varkappa_{2}, d_{1}, d_{2}\right)} \\
& +\frac{1}{\delta_{2}} \sqrt{\mathcal{L}_{\ell_{1}, \ell_{2}, \wp_{1}, \wp_{2}}\left(\left(s_{1}-\varkappa_{1}\right)^{2}\left(s_{2}-\varkappa_{2}\right)^{4} ; \varkappa_{1}, \varkappa_{2}, d_{1}, d_{2}\right)} \\
& \left.+\frac{1}{\delta_{1} \delta_{2}} \mathcal{L}_{\ell_{1}, \ell_{2}, \wp_{1}, \wp_{2}}\left(\left(s_{1}-\varkappa_{1}\right)^{2}\left(s_{2}-\varkappa_{2}\right)^{2} ; \varkappa_{1}, \varkappa_{2}, d_{1}, d_{2}\right)\right] .
\end{aligned}
$$

Since for $\left(\varkappa_{1}, \varkappa_{2}\right),\left(s_{1}, s_{2}\right) \in \mathbb{R}_{+}^{2}$ and $i=1,2 \mathcal{L}_{\ell_{1}, \ell_{2}, \wp_{1}, \wp_{2}}\left(\left(s_{1}-\varkappa_{1}\right)^{2 i}\left(s_{2}-\varkappa_{2}\right)^{2 j} ; \varkappa_{1}, \varkappa_{2}, d_{1}, d_{2}\right)$,

$$
=\mathcal{L}_{\ell_{1}, \ell_{2}, \wp_{1}, \wp_{2}}\left(\left(s_{1}-\varkappa_{1}\right)^{2 i} ; \varkappa_{1}, \varkappa_{2}, d_{1}, d_{2}\right) \mathcal{L}_{\ell_{1}, \ell_{2}, \wp_{1}, \wp_{2}}\left(\left(s_{2}-\varkappa_{2}\right)^{2 j} ; \varkappa_{1}, \varkappa_{2}, d_{1}, d_{2}\right),
$$

using Remark 4.3 and choosing $\delta_{1}=\frac{1}{\sqrt{\ell_{1}}}$ and $\delta_{2}=\frac{1}{\sqrt{\ell_{2}}}$, we reach the required result. 


\section{Numerical results and discussions}

We demonstrate and compare the rate of convergence of the GBS operators $\mathcal{G}_{\ell_{1}, \ell_{2}, \wp_{1}, \wp_{2}}\left(\varphi ; \varkappa_{1}, \varkappa_{2}, d_{1}, d_{2}\right)$ with the corresponding bivariate operators for the same function $\varphi\left(\varkappa_{1}, \varkappa_{2}\right)$ as considered in Example 5.1, with the same values of parameters.

Example 8.1. Let $\varphi\left(\varkappa_{1}, \varkappa_{2}\right)=\varkappa_{1}^{2} \varkappa_{2}-\varkappa_{1} \varkappa_{2}^{3}, \ell_{1}=\ell_{2}=30, d_{1}=d_{2}=2$, and $\wp_{1}=\wp_{2}=1,2$, and 3. The convergence of GBS operators associated with the generalized bivariate Kantorovich-Szász-Charlier type operators $\mathcal{L}_{\ell_{1}, \ell_{2}, \wp_{1}, \wp_{2}}\left(\varphi ; \varkappa_{1}, \varkappa_{2}, d_{1}, d_{2}\right)$ towards the function $\varphi\left(\varkappa_{1}, \varkappa_{2}\right)$, is illustrated in Figure 4 . Also, the absolute error in the approximation at certain points in the interval $\left(\varkappa_{1}, \varkappa_{2}\right) \in \mathbb{R}^{2}$ is computed in Table 3. Denote $\mathcal{G} \mathcal{E}_{\ell_{1}, \ell_{2}, \wp_{1}}^{\mathrm{d}_{1}, \wp_{2}}\left(\varphi ; \varkappa_{1}, \varkappa_{2}\right)=\left|\mathcal{G}_{\ell_{1}, \ell_{2}, \wp_{1}, \wp_{2}}\left(\varphi ; \varkappa_{1}, \varkappa_{2}, \mathrm{~d}_{1}, \mathrm{~d}_{2}\right)-\varphi\left(\varkappa_{1}, \varkappa_{2}\right)\right|$, the error function of approximation by $\mathcal{G}_{\ell_{1}, \ell_{2}, \wp_{1}, \wp_{2}}\left(\varphi ; \varkappa_{1}, \varkappa_{2}, d_{1}, d_{2}\right)$ operators. The error of approximation is illustrated in Figure 5 . We notice that the error in the approximation of the function by the operators becomes smaller as $\wp_{1}$ and $\wp_{2}$ increase.

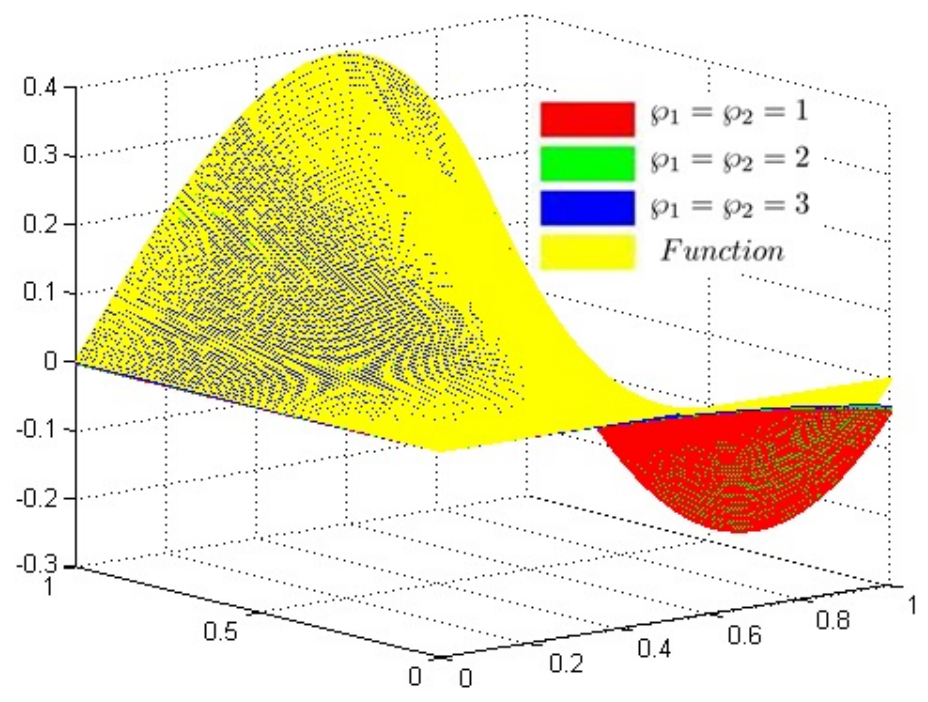

Figure 4: The convergence of operators $\mathcal{G}_{\ell_{1}, \ell_{2}, \wp_{1}, \wp_{2}}\left(\varphi ; \varkappa_{1}, \varkappa_{2}, \mathrm{~d}_{1}, \mathrm{~d}_{2}\right)$ to the function $\varphi\left(\varkappa_{1}, \varkappa_{2}\right)$.

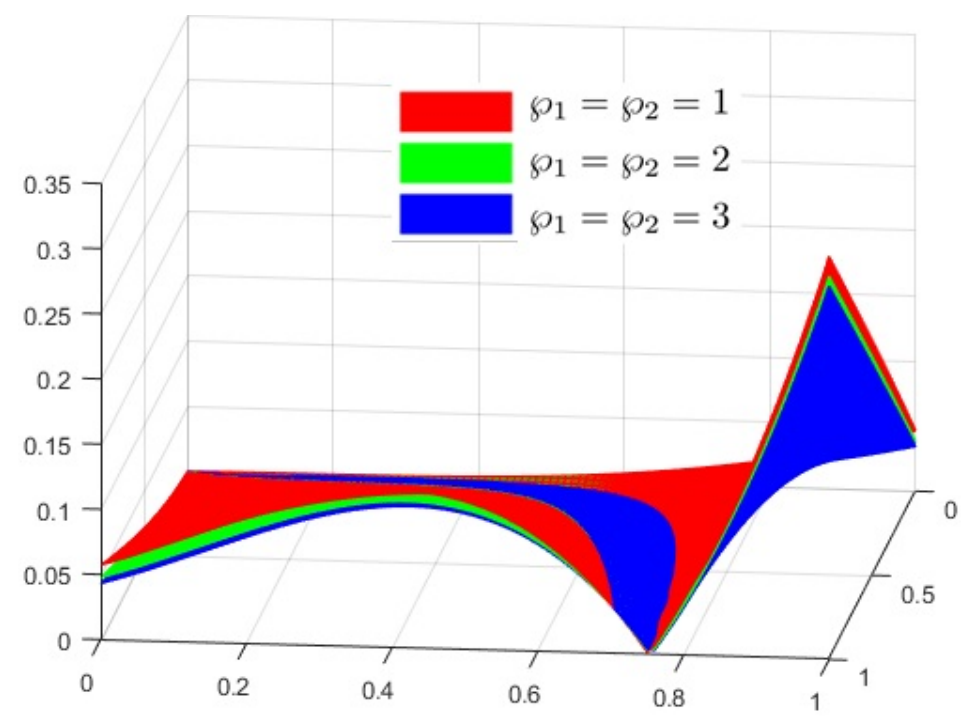

Figure 5: Error of approximation. 
Table 3: Error of approximation for operators $\mathcal{G}_{\ell_{1}, \ell_{2}, \wp_{1}, \wp_{2}}\left(\varphi ; \varkappa_{1}, \varkappa_{2}, \mathrm{~d}_{1}, \mathrm{~d}_{2}\right)$ to the function $\varphi\left(\varkappa_{1}, \varkappa_{2}\right)$.

\begin{tabular}{|c|c|c|c|}
\hline$\left(\varkappa_{1}, \varkappa_{2}\right)$ & $\mathcal{G} \mathcal{E}_{30,30,1}^{2,2,1}\left(\varphi ; \varkappa_{1}, \varkappa_{2}\right)$ & $\mathcal{G} \mathcal{E}_{30,30,2}^{2,2,2}\left(\varphi ; \varkappa_{1}, \varkappa_{2}\right)$ & $\mathcal{G} \mathcal{E}_{30,30,3}^{2,2,3}\left(\varphi ; \varkappa_{1}, \varkappa_{2}\right)$ \\
\hline$(0.1,0.1)$ & 0.0008 & 0.0006 & 0.0005 \\
\hline$(0.15,0.25)$ & 0.0016 & 0.0013 & 0.0012 \\
\hline$(0.20,0.35)$ & 0.0013 & 0.0008 & 0.0002 \\
\hline$(0.4,0.4)$ & 0.0040 & 0.0030 & 0.0022 \\
\hline$(0.5,0.55)$ & 0.0071 & 0.0058 & 0.0052 \\
\hline$(0.6,0.6)$ & 0.0082 & 0.0065 & 0.0057 \\
\hline$(0.85,0.9)$ & 0.0168 & 0.0124 & 0.0102 \\
\hline$(0.95,0.95)$ & 0.0167 & 0.0116 & 0.0091 \\
\hline
\end{tabular}

On comparing the Tables 2 and 3, we observe that the error of approximation $\mathcal{G E}_{\mathcal{\ell}_{1}, \ell_{2}, \wp_{1}}^{\mathrm{d}_{1}, \mathrm{~d}_{2}, \wp_{2}}\left(\varphi ; \varkappa_{1}, \varkappa_{2}\right)$ by the GBS operators $\mathcal{G}_{\ell_{1}, \ell_{2}, \wp_{1}, \wp_{2}}\left(\varphi ; \varkappa_{1}, \varkappa_{2}, d_{1}, d_{2}\right)$ to the function $\varphi\left(\varkappa_{1}, \varkappa_{2}\right)$ is smaller than the error $\mathcal{E}_{\ell_{1}, \ell_{2}, \wp_{1}}^{\mathrm{d}_{1} \mathrm{~d}_{2}, \wp_{2}}\left(\varphi ; \varkappa_{1}, \varkappa_{2}\right)$ by the corresponding bivariate operators $\mathcal{L}_{\ell_{1}, \ell_{2}, \wp_{1}, \wp_{2}}\left(\varphi ; \varkappa_{1}, \varkappa_{2}, \mathrm{~d}_{1}, \mathrm{~d}_{2}\right)$ to the function $\varphi\left(\varkappa_{1}, \varkappa_{2}\right)$. Hence, GBS operators yield us a better approximation to a certain function than the generalized bivariate Kantorovich-Szász-Charlier type operators.

\section{Acknowledgements}

The authors are extremely grateful to the learned reviewers for a critical reading of the paper and making valuable comments leading to a better presentation of the paper. The second author is thankful to "The Ministry of Human Resource and Development", India, for the financial support to carry out the above work.

\section{References}

[1] P. N. Agrawal, N. İspir, Degree of approximation for bivariate Chlodowsky-Szasz-Charlier type operators, Results. Math., 69 (2016), 369-385.

[2] C. Badea, K-Functionals and moduli of smoothness of functions defined on compact metric spaces, Comput. Math. Appl., 30 (1995), 23-31. 6

[3] C. Badea, I. Badea, H. Gonska, Notes on the degree of approximation of B-continuous and B-differentiable functions, J. Approx. Theory Appl., 4 (1988), 95-108. 6

[4] C. Badea, C. Cottin, Korovkin-type theorems for generalised Boolean sum operators, Approx. Theory (Kecskemat, Hungary), Colloq. Math. Soc. János Bolyai, 58 (1990), 51-67. 6

[5] D. Bărbosu, C. V. Muraru,Approximating B-continuous functions using GBS operators of Bernstein-Schurer-Stancu type based on q-integers, Appl. Math. Comput., 259 (2015), 80-87. 4

[6] K. Bögel, Mehrdimensionale Differentiation von Funktionen mehrerer reeller Veränderlichen, J. Reine Angew. Math., 170 (1934), 197-217. 6

[7] K. Bögel, Über mehrdimensionale Differentiation, Integration und beschränkte Variation, J. Reine Angew. Math., 173 (1935), 5-30. 6

[8] K. Bögel, Über die mehrdimensionale differentiation, Jber. Deutsch. Math.-Verein., 65 (1962), 45-71. 6

[9] P. L. Butzer, H. Berens, Semi-groups of Operators and Approximation, Springer-Verlag, New York, (1967). 4

[10] R. A. Devore, G. G. Lorentz, Constructive Approximation, Springer-Verlag, Berlin, (1993). 2

[11] Z. Ditzian, V. Totik, Moduli of Smoothness, Springer-Verlag, New York, (1987). 2, 4

[12] E. Dobrescu, I. Matei, The approximation by Bernštern type polynomials of bidimensionally continuous functions, An. Univ. Timişoara Ser. Şti. Mat.-Fiz., 4 (1996), 85-90. 6

[13] A. D. Gadzhiev, Theorems of Korovkin type, Math. Notes, 20 (1976), 995-998. 2, 2

[14] V. Gupta, T. M. Rassias, P. N. Agrawal, A. M. Acu, Recent Advances in Constructive Approximation Theorey, Springer, Cham, (2018). 6

[15] E. İbikli, E. A. Gadjiev, The order of approximation of some unbounded functions by the sequences of positive linear operators, Turkish J. Math., 19 (1995), 331-337. 2

[16] B. Lenze, On Lipschitz-type maximal functions and their smoothness spaces, Indag. Math., 91 (1988), 53-63. 2

[17] M. A. Özarslan, H. Aktuğlu, Local approximation properties for certain King type operators, Filomat, 27 (2013), $173-181$. 2 
[18] M. A. Özarslan, O. Duman, Smoothness properties of modified Bernstein-Kantorovich operators, Numer. Funct. Anal. Optim., 37 (2016), 92-105. 1

[19] J. Peetre, Theory of Interpolation of Normal Spaces, Lecture Notes, Brasilia, (1963).

[20] O. Szász, Generalization of S. Bernstein's polynomials to be infinite interval, J. Res. Natl. Bur. Stand., 45 (1950), $239-245$. 1

[21] B. S. Theodore, H. Hsu, A Non-linear Transformation for Sequences and Integrals, Thesis Master Sci, Graduate Faculty of Texas Thechnological College, (1968). 3

[22] S. Varma, F. Taşdelen, Szász type operators involving Charlier polynomials, Math. Comput. Model., 56 (2012), 118-122. $1,1,1.1$ 